\title{
Parametric upconversion imaging and its applications
}

Barh, Ajanta; Rodrigo, Peter John; Meng, Lichun; Pedersen, Christian; Tidemand-Lichtenberg, Peter

Published in:

Advances in Optics and Photonics

Link to article, DOI:

10.1364/aop.11.000952

Publication date:

2019

Document Version

Peer reviewed version

Link back to DTU Orbit

Citation (APA):

Barh, A., Rodrigo, P. J., Meng, L., Pedersen, C., \& Tidemand-Lichtenberg, P. (2019). Parametric upconversion imaging and its applications. Advances in Optics and Photonics, 11(4), 952-1019.

https://doi.org/10.1364/aop.11.000952

\section{General rights}

Copyright and moral rights for the publications made accessible in the public portal are retained by the authors and/or other copyright owners and it is a condition of accessing publications that users recognise and abide by the legal requirements associated with these rights.

- Users may download and print one copy of any publication from the public portal for the purpose of private study or research.

- You may not further distribute the material or use it for any profit-making activity or commercial gain

- You may freely distribute the URL identifying the publication in the public portal

If you believe that this document breaches copyright please contact us providing details, and we will remove access to the work immediately and investigate your claim. 


\title{
Parametric Upconversion Imaging and its Applications: A Review
}

\author{
Ajanta Barh, ${ }^{1, *}$ Peter John Rodrigo, ${ }^{2}$ Lichun Meng, ${ }^{2}$ Christian \\ Pedersen, ${ }^{2}$ And Peter Tidemand-Lichtenberg ${ }^{2, \dagger}$ \\ ${ }^{I}$ Institute of Quantum Electronics, Physics Department, ETH Zurich, 8093 Zurich, Switzerland \\ ${ }^{2}$ DTU Fotonik, Department of Photonics Engineering, Technical University of Denmark, 4000 Roskilde, \\ Denmark \\ “ajbarh@phys.ethz.ch; ‘ptli@fotonik.dtu.dk
}

\begin{abstract}
This article provides an extensive survey of nonlinear parametric upconversion infrared (IR) imaging, starting from its origin to date. Upconversion imaging is a successful innovative technique for IR imaging in terms of sensitivity, speed, and noise performance. In this approach, the IR image is frequency upconverted to form a visible/near-IR image through parametric three-wave mixing followed by detection using a silicon-based detector or camera. In 1968 (50 years back), J. E. Midwinter first demonstrated upconversion imaging from shortwave-IR $(1.6 \mu \mathrm{m})$ to visible $(484 \mathrm{~nm})$ wavelength using a bulk lithium niobate crystal. This technique quickly gained interest, and several other groups demonstrated upconversion imaging further into the mid- and far-IR with significantly improved quantum efficiency. Although a few excellent reviews on upconversion imaging were published in the early 1970's, the rapid progress in recent years merits an updated comprehensive review. The topic includes linear imaging, nonlinear optics, and laser science, and has shown diverse applications. The scope of this article is to provide an in-depth knowledge of upconversion imaging theory. An overview of different phase matching conditions for the parametric process and the sensitivity of the upconversion detection system are discussed. Furthermore, different design considerations and optimization schemes are outlined for application-specific upconversion imaging. The article comprises a historical perspective of the technique, its most recent technological advances, specific outstanding issues, and some cutting-edge applications of upconversion in IR imaging.

(C) 2019 Optical Society of America under the terms of the OSA Open Access Publishing Agreement
\end{abstract}

\section{TABLE OF CONTENTS}

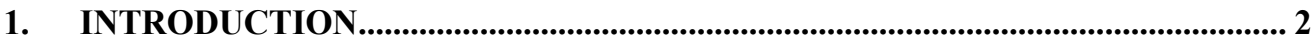

2. THEORY OF UPCONVERSION IMAGING .............................................................. 7

2.1 HELMHOLTZ EQUATION WITH A SOURCE TERM DUE TO A NONLINEAR MEDIUM......... 7

2.2 PLANE WAVE INTERACTION AND UPCONVERSION EFFICIENCY ................................ 9

2.3 WAVEVECTOR SPACE IMPULSE RESPONSE FUNCTION AND RESOLUTION IN THE

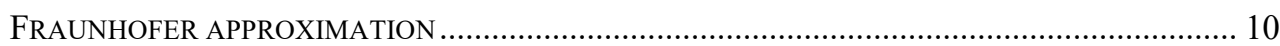

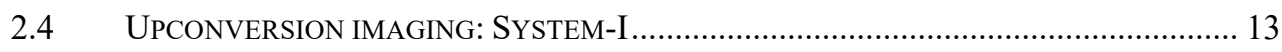

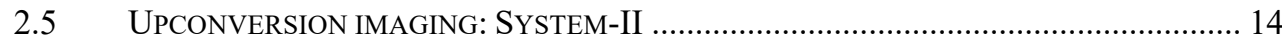

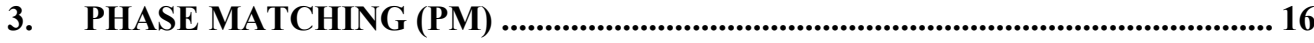

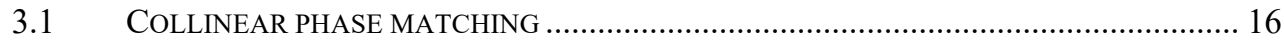

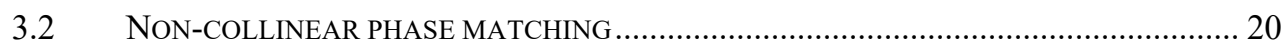

3.3 ACCEPTANCE PARAMETERS AND PHASE MATCH TUNING ........................................ 21

4. NOISE PROPERTIES OF UPCONVERSION.......................................................... 24 
5. DESIGN CONSIDERATIONS FOR UPCONVERSION IMAGING .27

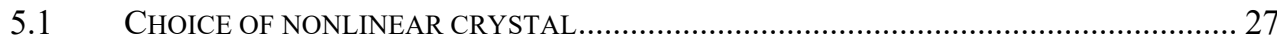

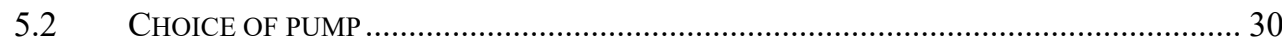

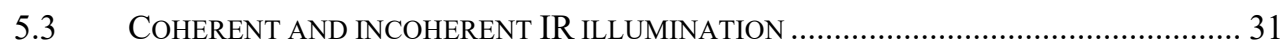

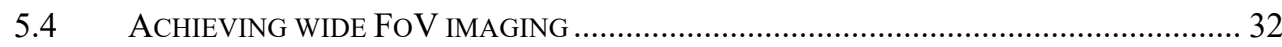

5.5 ANGULAR RESOLUTION AND NUMBER OF RESOLVABLE ELEMENTS ........................... 37

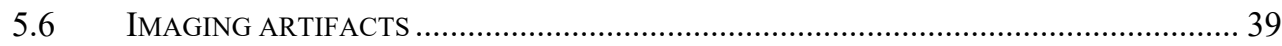

5.7 MULTI-DIMENSIONAL UPCONVERSION IMAGING ….............................................. 40

6. UPCONVERSION IMAGING APPLICATIONS .................................................... 42

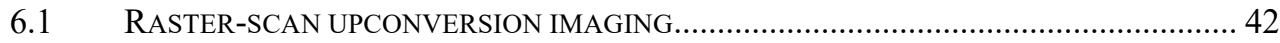

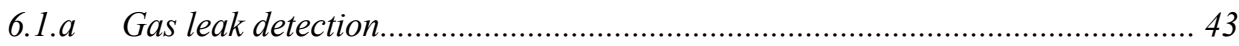

6.1.b Label-free breast cancer tissue diagnostics .................................................. 43

6.1.c MIR optical coherence tomography for non-destructive testing ....................... 44

6.2 VIDEO-RATE WIDE FOV UPCONVERSION IMAGING................................................... 45

6.2.a Label-free esophageal cancer tissue diagnostics ........................................... 46

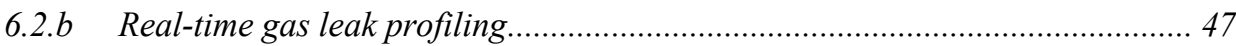

7. OTHER $\chi^{(2)}$ PARAMETRIC PROCESSES RELEVANT FOR IR IMAGING..... 47

8. CONCLUSION AND OUTLOOK ................................................................................... 51

9. FUNDING, ACKNOWLEDGMENTS, AND DISCLOSURES................................52

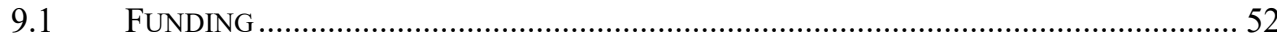

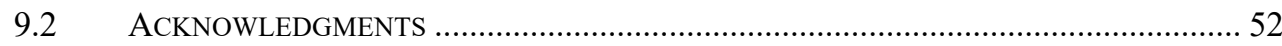

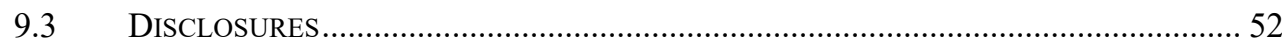

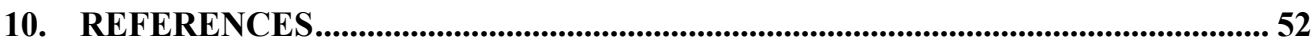

\section{Introduction}

Due to the rapid progress in optoelectronics that has matured the silicon CMOS/CCD technology, optical imaging in the visible (VIS) and near-infrared (NIR) spectral ranges (wavelength: $400-700 \mathrm{~nm}$ and $700-1100 \mathrm{~nm}$ ) has been the most successful one to date. However, there lies a wealth of information outside the VIS/NIR range that can advance our scientific knowledge and aid the development of new technologies. For example, over the last two decades, the mid-infrared (MIR: $2-15 \mu \mathrm{m}$ ) spectral range has attracted tremendous interest as it provides information-rich spectra that directly relates to the molecular structure of most bio-chemical materials [1]. This allows investigation of various materials in their near-native environment. This unique property is driving the development of cutting-edge tools for nondestructive medical diagnostics, food quality control, bacterial phase characterization, heavy gas sensing, monitoring of environmental trace gases and combustion gas flows, semiconductor processing, to name a few. This spectral range has several low loss atmospheric transmission windows in the $2-5 \mu \mathrm{m}$ and $8-14 \mu \mathrm{m}$ range, which are convenient for specialized atmospheric monitoring and terrestrial applications [2], [3]. This is further suitable for thermal imaging and defense applications [4]. All these applications (either based on passive or active illumination) rely on efficient, sensitive, and specific MIR detection/imaging systems. Current MIR direct detectors are primarily based on thermal sensors (e.g. microbolometer and pyroelectric) [5][8] or semiconductor based photon sensors (e.g. PbS, PbSe, HgCdTe, InAs, and InAsSb) [7], [9]. Thermal sensors are relatively cheaper; however, they are inherently slow in response 
$(\mathrm{kHz})$ and low in sensitivity, whereas semiconductor-based detectors can have high temporal bandwidth ( $\mathrm{MHz}$ to $\mathrm{GHz}$ ). The thermal radiation from the surrounding environment (temperature range: $0-200^{\circ} \mathrm{C}$ ) and of the detector itself peaks in the MIR range, which acts as background noise to the signal. Thus to separate the signal from this noise, often the semiconductor detectors are cooled using multi-stage thermoelectric coolers [9] or using liquid nitrogen at cryogenic temperature $\left(-195^{\circ} \mathrm{C}\right)$, and sealed to avoid temperature fluctuations. Such costly sophistication prohibits its widespread application. Furthermore, in both thermal and semiconductor based (direct) MIR sensors, the available pixel number is limited, i.e. in the order of $100 \times 100$. It renders the MIR image resolution much poorer for a given field-of-view (FoV) when compared to their VIS/NIR counterpart, where high quality CCD cameras are readily available.

A successful alternative scheme is parametric frequency upconversion. Instead of directly detecting in the IR, the IR signal is first parametrically transferred to the higher frequency (VIS/NIR) range by using a nonlinear optical process, which preserves (mostly) the spatial, temporal, and spectral information of the signal of interest. The converted signal is then detected by a standard sensitive CCD camera, now in a wavelength range that circumvents the room temperature thermal background noise. After the ground-breaking demonstration of the first laser in 1960 by Maiman [10], nonlinear optical processes were then discovered by exploiting the high intensity of a focused laser beam. In 1961, Franken et al. [11], first observed second harmonic generation (at $\sim 347.2 \mathrm{~nm}$ ) from a ruby laser (operating at $694.3 \mathrm{~nm}$ ) in a quartz crystal. As long as the nonlinear medium is not participating directly in the energy exchange process (between input and generated light), the process is widely known as parametric frequency conversion, and it works instantaneously. The term "upconversion" was first coined in 1962 by Armstrong et al. in their work [12], where they described "...if the objective is to detect a weak coherent light signal at $\omega_{\mathrm{s} . . . .}$ one may use to advantage parametric up-conversion by means of a powerful laser beam at the pump frequency $\omega_{\mathrm{p}}$. The output is taken at the sum frequency $\omega_{\mathrm{I}}=\omega_{\mathrm{s}}+\omega_{\mathrm{p}}$ in an up-converter." In this way, the IR information can be transferred instantly to the VIS/NIR range for final detection, ideally preserving the spatial, temporal, and spectral signatures encoded in the IR signal. Upconversion is a second order nonlinear process; whereas the successive orders of nonlinear processes are generally weaker and not within the scope of this review article. For higher (third) order nonlinearity, the reader may consider [13] for further details. Note that this review considers parametric upconversion, in which the medium is unchanged and only facilitates the conversion process, unlike in photon upconversion [14]-[16].

In 1962, Smith and Braslau [17] first demonstrated upconversion of a weak visible light signal using a strong ruby laser as pump in a KDP crystal. Due to limited availability of nonlinear crystals, it took another 5 years to demonstrate upconversion of an IR signal. Midwinter and Warner [18] demonstrated upconversion of $1.7 \mu \mathrm{m}$ radiation to $\sim 493 \mathrm{~nm}$ using a lithium niobate $\left(\mathrm{LiNbO}_{3}\right)$ crystal. They achieved $1 \%$ collinear conversion efficiency using a pump intensity of $1.9 \mathrm{MW} / \mathrm{cm}^{2}$ in a $1.35 \mathrm{~cm}$ long crystal. In 1968, Midwinter [19] first demonstrated parametric upconversion imaging from short-wave-IR (Xenon lamp at $1.6 \mu \mathrm{m}$ ) to VIS (484.2 $\mathrm{nm}$ ) using a $\mathrm{LiNbO}_{3}$ crystal for upconversion and a photomultiplier for detection. He achieved a spatial resolution of 50 lines across a $60 \mathrm{mrad}$ entrance aperture with upconversion efficiency in the order of $10^{-7}$. This technique quickly gained interest, and in subsequent years, several research groups demonstrated upconversion imaging from IR to VIS/NIR range [20]-[27] and detailed theoretical studies dealing with this imaging technique were published [28]-[33]. In the 1970's, this research field reached a peak, but was afterwards almost abandoned for three decades. Possible reasons for the lack of progress of the technology during these decades may include; (a) limited sensitivity of available photocathodes and immaturity of CCD detectors, (b) limited choice and poor quality of nonlinear crystals, (c) that only pulsed laser was available for high power pumping, which is not very efficient for upconverting a CW IR signal, (d) that intracavity based CW upconversion was in its infancy, 
i.e. high power intracavity systems were challenging mainly due to high optical losses associated with multiple passes through intracavity crystals, and finally (e) that the technology was there but the target application was not clear or broad enough. With the rapid progress in silicon technology (sensitive CCDs), successful growth of impurity-free nonlinear crystals of various types, smart design of upconversion systems, availability of high-power MIR sources for active illumination, and demonstrations of potential applications including those within interdisciplinary fields, upconversion imaging re-gained its momentum from the middle of 2000 's. The focus of the renewed interest in upconversion imaging included works to enhance image FoV, improve spatial resolution, increase conversion efficiency, reduce noise, lower cost of operation, and demonstrate real life applications. Table 1 shows the time-line for different historical events which have had significant impact on the progress of the upconversion based IR imaging technique.

Table 1. Historical Time-line of Notable Scientific Accomplishments Relevant to the Progress of Upconversion Imaging

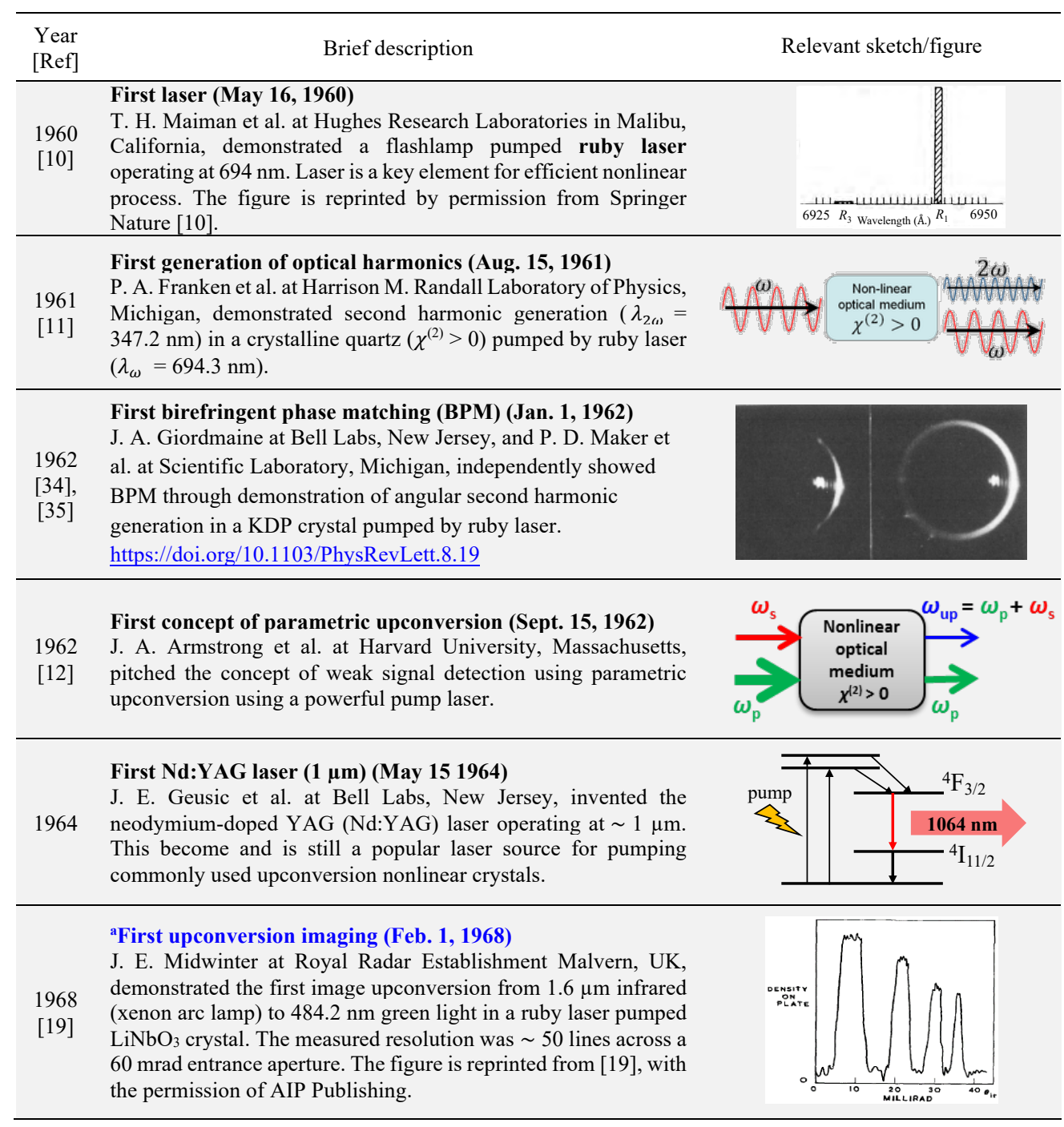




\begin{tabular}{|c|c|c|}
\hline $\begin{array}{l}1970 \\
{[36]}\end{array}$ & $\begin{array}{l}\text { Invention of charge-coupled device (CCD) (Apr. 1970) } \\
\text { W. S. Boyle and G. E. Smith at Bell Labs first published the } \\
\text { invention of CCD on a silicon-CMOS platform, which is the key } \\
\text { technology of today's CCD cameras for visible-NIR detection. } \\
\text { The figure is adapted from [36]. }\end{array}$ & 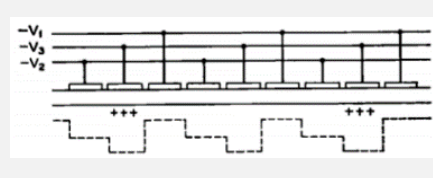 \\
\hline $\begin{array}{l}1971 \\
{[37]}\end{array}$ & \multicolumn{2}{|c|}{$\begin{array}{l}\text { Upconversion detection of room-temperature blackbody (Aug. 1, 1971) } \\
\text { J. Falk and J. M. Yarborough at GTE Sylvania Inc., California, first demonstrated upconversion of a room- } \\
\text { temperature thermal radiation }(6.5-12.5 \mu \mathrm{m}) \text { in a Nd:YAG laser pumped proustite crystal. This is } \\
\text { important for thermal imaging. }\end{array}$} \\
\hline $\begin{array}{l}1976 \\
{[38],} \\
{[39]}\end{array}$ & $\begin{array}{l}\text { Quasi phase matching (QPM) (May 1976, Aug. 1976) } \\
\text { A. Szilagyi et al. at Air Force Cambridge Research Laboratories, } \\
\text { Massachusetts, and M. S. Piltch et al. at University of California, } \\
\text { New Mexico, independently demonstrated, for the first time, } \\
\text { frequency conversion using the QPM concept. Both the groups } \\
\text { used a stack of non-birefringent crystal plates (GaAs by former } \\
\text { and } \mathrm{CdTe} \text { by later group) in Brewster angle to frequency convert } \\
\text { the } \mathrm{CO}_{2} \text { laser at } 10.6 \mu \mathrm{m} \text {. The figure is reprinted from [39], with } \\
\text { the permission of AIP Publishing. }\end{array}$ & \\
\hline $\begin{array}{l}1978 \\
{[40]}\end{array}$ & $\begin{array}{l}\text { Intracavity upconversion (Jan. 15, 1978) } \\
\text { J. Falk and Y. C. See at University of Pittsburgh, Pennsylvania, } \\
\text { first demonstrated intracavity upconversion for detection of a } \\
\text { continuous wave }\left(\mathrm{CW} \text { ) signal. A } \mathrm{LiNbO}_{3} \text { crystal of good quality }\right. \\
\text { (low loss) was placed inside an argon-ion laser cavity (pump, } \\
514.5 \mathrm{~nm} \text { ) to upconvert a CW } 3.39 \mu \mathrm{m} \text { He-Ne laser. }\end{array}$ & $\begin{array}{l}\text { Image of a } \\
\text { state-of-the- } \\
\text { art } \mathrm{LiNbO}_{3} \\
\text { crystal }\end{array}$ \\
\hline $\begin{array}{l}1994 \\
{[41]}\end{array}$ & $\begin{array}{l}\text { Quantum cascade laser (QCL) (Apr. 22, 1994) } \\
\text { J. Faist et al. at Bell Labs, New Jersey, first demonstrated a new } \\
\text { type of semiconductor laser (QCL) enabling chip-scale laser } \\
\text { source in the mid- to far-infrared range. They demonstrated a } \\
\text { QCL operating at } 4.2 \mu \mathrm{m} \text {. Such lasers are suitable for chip-scale } \\
\text { active illumination based infrared detection. The figure is from } \\
\text { [41]. Reprinted with permission from AAAS. }\end{array}$ & to \\
\hline $\begin{array}{l}1995 \\
{[42],} \\
{[43]}\end{array}$ & $\begin{array}{l}\text { Imaging via optical parametric amplification (OPA) } \\
\text { F. Devaux et al. at Universite de Franche-Comte, France, first demo } \\
\text { object illuminated by a Nd:YAG laser using KTP as nonlinear cryst }\end{array}$ & $\begin{array}{l}1 \text { strated OPA based imaging of an } \\
1 \text { and CCD camera for detection. }\end{array}$ \\
\hline 2000 & $\begin{array}{l}\text { From the beginning of the } 21^{\text {st }} \text { century, the Si-CMOS industry has s } \\
\text { to high quality (sensitive, large pixel density) CCD chips in the } n \\
\text { upconversion imaging. }\end{array}$ & $\begin{array}{l}\text { rowth that has led } \\
\text { the sensitivity of }\end{array}$ \\
\hline $\begin{array}{l}2007 \\
{[44]}\end{array}$ & $\begin{array}{l}\text { aChemical/spectral upconversion imaging (July 1, 2007) } \\
\text { M. Imaki and T. Kobayashi at University of Fukui, Japan, } \\
\text { demonstrated an application of upconversion imaging in } \\
\text { chemical sensing (methane detection) at } 3.4 \mu \mathrm{m} \text {. Their } \\
\text { upconversion detector's sensitivity was } 11 \text { times better than a } \\
\text { reference direct detector (cooled InAs). The image is adapted } \\
\text { with permission from [44] C The Optical Society. }\end{array}$ & $\begin{array}{l}\text { Image of } \\
\text { methane gas } \\
\text { plume }\end{array}$ \\
\hline $\begin{array}{l}2012 \\
{[45]}\end{array}$ & 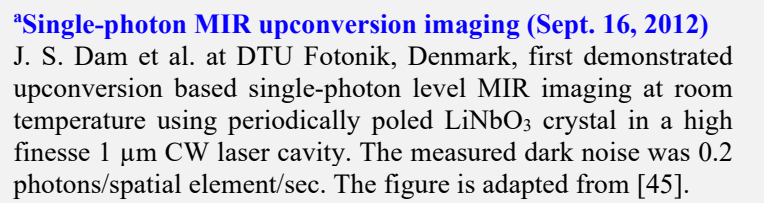 & $\begin{array}{l}\text { White } \\
\text { dots are } \\
\text { detected } \\
\text { photons }\end{array}$ \\
\hline $\begin{array}{c}2014 \\
{[46]}\end{array}$ & $\begin{array}{l}\text { Ultrawide MIR supercontinuum source (Sept. 14, 2014) } \\
\text { C. Petersen et al. at DTU Fotonik, Denmark, first demonstrated } \\
\text { an optical fiber-based supercontinuum source covering } 1.4-13.3 \\
\mu \mathrm{m} \text { MIR range. Such a table-top high-brightness MIR source is } \\
\text { suitable for active illumination based broadband upconversion } \\
\text { imaging. Reprinted by permission from [46]. }\end{array}$ & \\
\hline
\end{tabular}




\begin{tabular}{|c|c|c|}
\hline $\begin{array}{l}2015 \\
{[47]}\end{array}$ & $\begin{array}{l}\text { a Hyperspectral upconversion imaging (Mar. 15, 2015) } \\
\text { L. M. Kehlet et al. at DTU Fotonik, Denmark, demonstrated } \\
\text { hyperspectral imaging of a test target combined with a } \\
\text { polystyrene (PS) film in the } 3.2-3.4 \mu \mathrm{m} \text { range, by detecting the } \\
\text { upconverted image using a Si-CCD camera. The figure is adapted } \\
\text { from [47]. }\end{array}$ & $\begin{array}{l}\text { PS } \\
\text { absorption } \\
\text { in } 3.2- \\
3.4 \mu \mathrm{m}\end{array}$ \\
\hline
\end{tabular}

ademonstrations that directly rely on upconversion based IR imaging.

Current state-of-the-art technologies for direct IR detection are mainly based on thermal detectors [48] (low cost, but slow and have low sensitivity), semiconductor detectors (highly sensitive, but need special cooling and sophisticated handling), or superconducting nanowire detectors [49] (highly sensitive and fast, but work at extremely low temperature, i.e. few $\mathrm{mK}$ to $\mathrm{K}$ ). Researchers have compared the sensitivity of upconversion detectors with direct detectors at different IR operating wavelengths [44], [50]-[54], however, upconversion detectors are still to be optimized for a given application to reach optimal performance.

This review article mainly focuses on upconversion imaging. Although there are a few excellent reviews published in 1970's [55]-[59], the rapid progress in upconversion imaging in the $21^{\text {st }}$ century (Fig. 1) is seen as a motivating factor for considering an updated comprehensive review. Point detection-based spectroscopy lies outside the scope of this review. Thus, mainly bulk crystals (not wave guiding structures) are considered as the nonlinear medium where noncollinear wave interaction can be accessed to achieve wide FoV imaging with multiple spatially resolved elements. This is an extensive review wherein fundamental working principles are discussed along with the reported results including cutting-edge research and outstanding issues. The activity on upconversion imaging as a research field is illustrated in Fig. 1 using the number of Web of Science indexed publications (only in journals and conference proceedings) per year to date.

This article is divided into six main sections. In the next section (section 2), the general principle/theory of upconversion imaging is described starting from classical Helmholtz equation with nonlinear source term. The solution is established at far field for phase matched plane wave interaction with non-depleted pump. Consequently, an expression for the upconversion efficiency is derived. Using the special case of a Gaussian pump, the upconversion imaging and its resolution is described using the impulse response function in wavevector space. The section is concluded with a description of two primary types of upconversion imaging system, namely upconversion in Fourier plane and upconversion in image plane. In the next section (section 3), a detailed analysis of phase matching conditions in $\chi^{(2)}$ nonlinear materials are discussed, starting from collinear wave interaction to non-collinear case. The section is concluded with a description on acceptance bandwidth and tunability of the phase matching condition. The next section (section 4) is devoted to analyze different noise sources in an upconversion process, which is important to compare the sensitivity and suitability of upconversion imaging system in comparison to direct MIR imaging. After discussing all the basics of upconversion imaging, various design parameters are reviewed in the next section (section 5), which includes choice of initial parameters (pump, nonlinear crystal, coherent/incoherent IR illumination), optimization of imaging FoV and resolution etc. The section is concluded by outlining upconversion based multi-dimensional imaging, especially highlighting hyperspectral imaging. In section 6 , recent applications of parametric upconversion imaging exploiting both raster-scanning scheme and 2-dimensional (2-D) sensorbased detection scheme are described. In the last section (section 7), we mention possibilities of other $\chi^{(2)}$ nonlinear processes based (not upconversion) imaging schemes. However, as it lies outside the scope of the current review article, we keep it brief. The article is concluded with a brief discussion on the outlook and trends of upconversion imaging. 


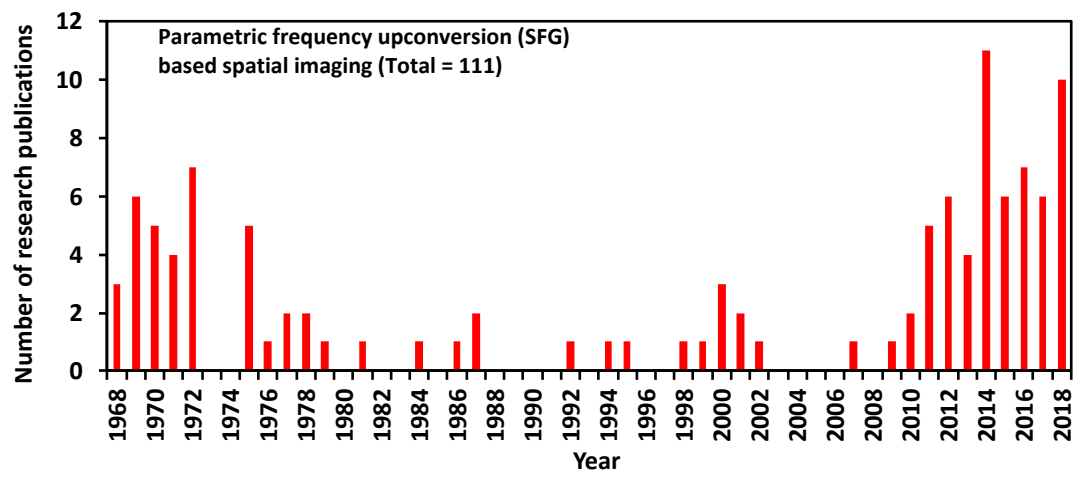

Fig. 1. Number of publications (journal + conference proceedings) on upconversion spatial imaging in each year since 1968. Note that, the key words for searching the publications are: parametric, nonlinear, frequency, conversion, upconversion, spatial, imaging. In addition, a manual filtering of relevant papers related to parametric frequency upconversion (sum frequency generation) based spatial imaging is considered. Search engine: Web of Science, Latest considered publication date: 31 December 2018.

\section{Theory of upconversion imaging}

The three interacting waves in the frequency upconversion process are denoted by subscripts $p$ (pump), IR (input infrared signal), and up (generated upconverted). Bold letters are primarily used for vector notation. Scalar wavenumbers in italic are used for the wavevector components.

\subsection{Helmholtz equation with a source term due to a nonlinear medium}

Nonlinear interaction based on $\chi^{(2)}$ is a standard textbook material [60]-[62] when describing frequency conversion due to interactions of Gaussian laser beams, however, the generalized version involving two arbitrary electromagnetic fields (for e.g., IR signal and pump) is less well-known. In order to treat upconversion imaging in which the object is represented by a spatially complex electromagnetic field distribution, a theory that covers diffraction and a high imbalance among the interacting fields is needed. This section describes a practical way to translate classical diffraction theory to the nonlinear $\chi^{(2)}$ case using a number of simplifying assumptions. Foremost, we adopt the non-depletion (small signal) approximation, thus excluding situations where the upconverted field modifies the source term. Secondly, since the interaction often takes place inside a birefringent crystal including extraordinary polarized $(e)$ waves, walk-off may be present. In order not to complicate the treatment here beyond its purpose, we ignore this often small effect assuming large beam diameters and/or short crystal lengths. Walk-off can be included if needed [63], [64]. Thirdly, for convenience, we assume that the nonlinear medium is submerged in a medium of the same refractive index to focus the discussion to the nonlinear interaction inside the crystal.

Classical scalar diffraction theory is based on the 3-dimensional (3-D) Helmholtz equation, which for the nonlinear case has a non-zero source term [65]:

$$
\nabla^{2} E_{\text {up }}(\mathbf{r})+k_{\text {up }}^{2} E_{\text {up }}(\mathbf{r})=-S_{\text {up }}(\mathbf{r}) .
$$

$k_{\text {up }}$ is the wavenumber for the upconverted field, $E_{\text {up }}$. For the nonlinear $\chi^{(2)}$ case, the source term, $S_{\text {up }}$, originates from a non-vanishing effective second order susceptibility, $d_{\text {eff }}$, in the presence of two incident monochromatic external fields. Without loss of generality, these two fields can be expressed as: $E_{\mathrm{IR}}(\mathbf{r}, t)=A_{\mathrm{IR}}(\mathbf{r}) e^{j\left(\mathbf{k}_{\mathrm{IR}} \cdot \mathbf{r}-\omega_{\mathrm{IR}} t\right)}$ and $E_{\mathrm{p}}(\mathbf{r}, t)=A_{\mathrm{p}}(\mathbf{r}) e^{j\left(\mathbf{k}_{\mathrm{p}} \cdot \mathbf{r}-\omega_{\mathrm{p}} t\right)}$ with wavevectors $\mathbf{k}_{\mathrm{IR}}$ and $\mathbf{k}_{\mathrm{p}}$, and angular frequencies, $\omega_{\mathrm{IR}}$ and $\omega_{\mathrm{p}}$, respectively (see Fig. 2). This 
specific representation of the fields, where the spatial carrier frequencies are singled out is included to underline the phase matching aspect introduced later in this section, which impose strict requirements for efficient field interaction. Due to energy conservation, the angular frequency of the upconverted field needs to satisfy the following:

$$
\omega_{\mathrm{up}}=\omega_{\mathrm{IR}}+\omega_{\mathrm{p}}
$$

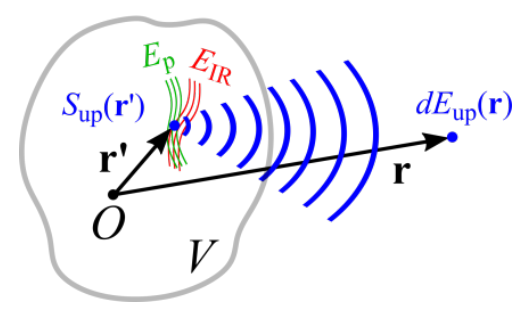

Fig. 2. Schematic diagram used to calculate the upconverted field $E_{\text {up }}$ at a position given by the vector $\mathbf{r}$ relative to a point of origin $O$ inside a nonlinear $\chi^{(2)}$ medium of volume $V$.

Equation (2) demonstrates a $\chi^{(2)}$ nonlinear crystal's ability to translate a MIR signal from $\omega_{\mathrm{IR}}$ to a higher angular frequency $\omega_{\mathrm{up}}$ in the presence of a pump field at $\omega_{\mathrm{p}}$. The source term for the upconverted field is given by [65]:

$$
S_{\text {up }}(\mathbf{r})=\frac{2 d_{\text {eff }} \omega_{\text {up }}^{2}}{c^{2}} A_{\mathrm{IR}}(\mathbf{r}) A_{\mathrm{p}}(\mathbf{r}) e^{j\left(\mathbf{k}_{\mathrm{IR}}+\mathbf{k}_{\mathrm{p}}\right) \cdot \mathbf{r}},
$$

where $d_{\text {eff }}$ (in $\mathrm{m} /$ volt units) depends on the specific interaction scheme between the fields and $c$ is the speed of light in vacuum. Assuming that $E_{\text {up }}$ satisfies both homogeneous Dirichlet and homogeneous Neumann boundary conditions, an explicit analytical solution to Eq. (1) is given by Green's function approach [66]:

$$
E_{\text {up }}(\mathbf{r})=\int_{V} G_{3}\left(\mathbf{r}, \mathbf{r}^{\prime}\right) S_{\text {up }}\left(\mathbf{r}^{\prime}\right) d \mathbf{r}^{\prime},
$$

where the Green function is given by:

$$
G_{3}\left(\mathbf{r}, \mathbf{r}^{\prime}\right)=\frac{e^{j k_{\mathrm{up}}\left|\mathbf{r}-\mathbf{r}^{\prime}\right|}}{4 \pi\left|\mathbf{r}-\mathbf{r}^{\prime}\right|}(\text { for the 3-D case). }
$$

Thus,

$$
E_{\text {up }}(\mathbf{r})=\frac{2 d_{\text {eff }} \omega_{\text {up }}^{2}}{c^{2}} \int_{V} \frac{e^{j k_{\mathrm{up}}\left|\mathbf{r}-\mathbf{r}^{\prime}\right|}}{4 \pi\left|\mathbf{r}-\mathbf{r}^{\prime}\right|} A_{\mathrm{IR}}\left(\mathbf{r}^{\prime}\right) A_{\mathrm{p}}\left(\mathbf{r}^{\prime}\right) e^{j\left(\mathbf{k}_{\mathrm{IR}}+\mathbf{k}_{\mathrm{p}}\right) \cdot \mathbf{r}^{\prime}} d \mathbf{r}^{\prime},
$$

where the integration is over a volume $V$ (see Fig. 2). The solution is a mathematical formulation of Huygens-Fresnel principle for a $\chi^{(2)}$ material.

It is clear from Fig. 2 that when an observation point is in close proximity of the source, different point sources contribute to $E_{\text {up }}(\mathbf{r})$ with different $\left|\mathbf{r}-\mathbf{r}^{\prime}\right|$ and may propagate with different refractive indices as in the case of birefringent crystals. For a birefringent crystal, the refractive index for an $e$-ray is a function of propagation angle [61]. However, without providing a full mathematical proof, it is still possible to formulate Huygens-Fresnel principle 
in the case of birefringent crystals, associating the refractive index $n_{\text {up }}$ with each point source, at $\mathbf{r}^{\prime}$, in the material. Assuming that $n_{\text {up }}$ varies linearly with angle of propagation (first linear term in a Taylor expansion of $n_{\text {up }}$ ), it can be shown from calculus of variation that an $e$-ray indeed follows the direct path between a source point and the observation point, thus maintaining the notion of $n_{\text {up }}\left|\mathbf{r}-\mathbf{r}^{\prime}\right|$ as the shortest optical path length - see first exponential term of Eq. (6) and Fig. 2. Walk-off effects, if relevant, are usually dealt with by a linear translation of the source point relative to the observation point, dictated by the walk-off angle [63], [67]. To summarize, the similarity with classical scalar diffraction theory allows us to extensively use the vast theory already developed [68].

\subsection{Plane wave interaction and upconversion efficiency}

The appropriate Green function is different in one, two and three dimensions. To illustrate this point and to provide a simple expression for the quantum efficiency $(\mathrm{QE})$ of the upconversion process, we consider the 1-dimensional (1-D) case for plane wave interaction along the $z$-axis, in a nonlinear crystal of length $l_{c}$ (see Fig. 3). The signal and pump fields are given by

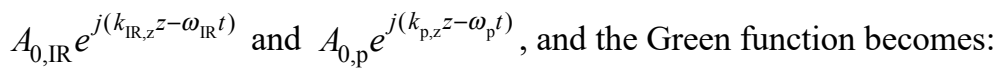

$$
G_{1}\left(z, z^{\prime}\right)=\frac{j e^{j k_{\mathrm{up}, z}\left|z^{\prime}\right|}}{2 k_{\mathrm{up}, \mathrm{z}}} \quad(\text { for the 1-D case). }
$$

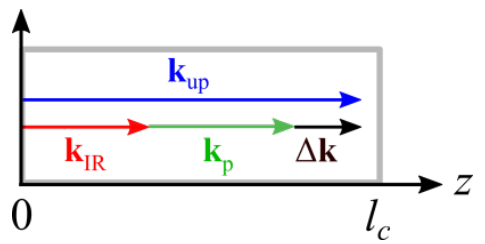

Fig. 3. Schematic diagram used to calculate the upconverted field $E_{\text {up }}$ at the end facet of a rectangular nonlinear crystal of length $l_{c}$ and the IR signal and pump fields are plane waves propagating in the $+z$-direction ( 1 -D case).

Thus, the upconverted field at the end facet of the nonlinear crystal is given by:

$$
\begin{aligned}
E_{\mathrm{up}}\left(z=l_{c}\right)=\left.\int G_{1}\left(z, z^{\prime}\right) S_{\mathrm{up}}\left(z^{\prime}\right) d z^{\prime}\right|_{z=l_{c}} & =\int_{0}^{l_{c}} \frac{j e^{j k_{\mathrm{upp},}\left(l_{c}-z^{\prime}\right)}}{2 k_{\mathrm{up}, z}} \frac{2 d_{\mathrm{eff}} \omega_{\mathrm{up}}^{2} A_{0, \mathrm{p}} A_{0, \mathrm{R}} e^{j\left(k_{\mathrm{IR}, z}+k_{\mathrm{p}, z}\right) z^{\prime}}}{c^{2}} d z^{\prime} \\
& =\frac{j d_{\mathrm{eff}} l_{c} A_{0, \mathrm{p}} A_{0, \mathrm{IR}} \omega_{\mathrm{up}} e^{j k_{\mathrm{up}, z} l_{c}} e^{-j \Delta k_{z} l_{c} / 2}}{n_{\mathrm{up}} c} \operatorname{sinc}\left(\frac{\Delta k_{z} l_{c}}{2}\right)
\end{aligned}
$$

$\Delta k_{\mathrm{z}}=k_{\mathrm{up}, \mathrm{z}}-k_{\mathrm{IR}, \mathrm{z}}-k_{\mathrm{p}, \mathrm{z}}$, where subscript " $z$ " refers to the $z$-direction. $\Delta k_{\mathrm{z}}$ is referred to as the phase mismatch along the $z$-axis. If $\Delta k_{\mathrm{z}}=0$, the upconversion process is termed "phase matched" along the $z$-axis.

From Eq. (8), the upconverted intensity can be expressed as:

$$
\begin{aligned}
I_{\text {up }}\left(l_{c}\right)=\frac{\varepsilon_{0} n_{\text {up }} c}{2}\left|E_{\text {up }}\right|^{2} & =\frac{\varepsilon_{0} d_{\mathrm{eff}}^{2} l_{c}^{2} A_{0, \mathrm{p}}^{2} A_{0, \mathrm{IR}}^{2} \omega_{\mathrm{up}}^{2}}{2 n_{\mathrm{up}} c} \operatorname{sinc}^{2}\left(\frac{\Delta k_{\mathrm{z}} l_{c}}{2}\right) \\
& =\frac{8 \pi^{2} d_{\mathrm{eff}}^{2} l_{c}^{2} I_{0, \mathrm{p}} I_{0, \mathrm{IR}}}{\varepsilon_{0} c n_{\mathrm{IR}} n_{\mathrm{p}} n_{\mathrm{up}} \lambda_{\mathrm{up}}^{2}} \operatorname{sinc}^{2}\left(\frac{\Delta k_{\mathrm{z}} l_{c}}{2}\right),
\end{aligned}
$$


where angular frequencies have been expressed in terms of corresponding wavelengths $(\lambda)$ and refractive indices $(n) . I_{0, \mathrm{IR}}$ and $I_{0, \mathrm{p}}$ are the intensities of the IR signal and pump fields at the input, respectively.

Thus, the power conversion efficiency is defined as:

$$
\tilde{\eta}_{\text {up }}=\frac{I_{\text {up }}\left(l_{c}\right)}{I_{0, \mathrm{IR}}}=\frac{8 \pi^{2} d_{\mathrm{eff}}{ }^{2} l_{c}^{2}}{\varepsilon_{0} c n_{\mathrm{IR}} n_{\mathrm{p}} n_{\text {up }} \lambda_{\mathrm{up}}^{2}} \frac{P_{\mathrm{p}}}{A} \operatorname{sinc}^{2}\left(\frac{\Delta k_{\mathrm{z}} l_{c}}{2}\right),
$$

where $A$ is the area of either the pump beam or the aperture of the nonlinear crystal, whichever is smaller, and $P_{\mathrm{p}}$ is the pump power contained in $A$. Note that Eq. (10) is valid only for plane wave interactions. In bulk nonlinear crystals, the validity holds for interacting Gaussian fields with relatively large beam radii (i.e., have Rayleigh lengths larger than $l_{c}$ ). This sets a lower bound to $A$ if it is defined by the pump beam waist. In waveguides embedded in nonlinear crystals, which support single transverse modes of the interacting fields, smaller $A$ values are possible over the full length of the waveguide. Therefore, for most cases where waveguide crystals are used, the non-depletion approximation is invalid [61].

The expression in Eq. (10) for the power conversion efficiency under non-depletion approximation is identical to that found in standard treatments of nonlinear optics, however, the latter is based on coupled differential equations [61]. Several simple observations can be drawn from $\tilde{\eta}_{\text {up }}$. The power conversion efficiency is independent of the incident radiation $I_{0, \mathrm{IR}}$ , thus, extremely weak signals can be upconverted with the same efficiency as relatively larger IR signals. This is a key property of upconversion imaging. It also means that the IR signal dynamic range is extremely large ranging from single photons to Watt level signals. Secondly, the efficiency is inversely proportional to $A$. However, decreasing $A$ acts adversely on the spatial resolution (as discussed in sub-section 2.4), thus constituting a dilemma when designing an upconversion imaging system. A third aspect relates to $\Delta k_{z} l_{c}$, i.e., the phase mismatch between the three interacting waves. As $\Delta k_{z} l_{c}$ deviates from zero (i.e., phase mismatched), the efficiency decreases dramatically. This property is not present in linear imaging systems and is of major concern for upconversion imaging. In section 3, phase matching conditions using birefringent crystals are discussed in more detail. The quantum efficiency of the upconversion process (or upconversion efficiency, for brevity) is given by $\eta_{\text {up }}=\tilde{\eta}_{\text {up }} \times\left(\lambda_{\text {up }} / \lambda_{\mathrm{IR}}\right)$ which is the internal quantum efficiency. Since the upconverted wave is followed by a VIS/NIR detector (e.g. Si-CCD), the detector quantum efficiency $\left(\eta_{\text {det }}\right)$, as well as an additional factor coming from other external losses $\left(\eta_{\text {extloss }}\right)$, must be included to calculate the overall quantum efficiency of the upconversion detection system (also referred to as overall detection efficiency):

$$
\eta_{\text {system }}=\eta_{\text {up }} \times \eta_{\text {det }} \times \eta_{\text {ext-loss }} .
$$

\subsection{Wavevector space impulse response function and resolution in the Fraunhofer approximation}

We will in the following concentrate on observation points located in the far-field $|\mathbf{r}| \gg\left|\mathbf{r}^{\prime}\right|$ (see Fig. 2) such that all source-generated fields that are coherently superimposed at a single observation point given by $\mathbf{r}$ propagate in the same direction, experiencing the same refractive index. Using the approximations $k_{\text {up }}\left|\mathbf{r}-\mathbf{r}^{\prime}\right| \approx k_{\text {up }} r-\mathbf{k}_{\text {up }} \cdot \mathbf{r}^{\prime}$ and $\left|\mathbf{r}-\mathbf{r}^{\prime}\right| \approx|\mathbf{r}|=r$ in the numerator 
and denominator of $G_{3}$, respectively, a simpler expression for the upconverted field can be derived from Eq. (6):

$$
\begin{aligned}
E_{\mathrm{up}}(r) & =\frac{d_{\mathrm{eff}} \omega_{\mathrm{up}}^{2}}{c^{2}} \frac{e^{j k_{\mathrm{up}}{ }^{r}}}{2 \pi r} \int_{V} A_{\mathrm{IR}}\left(\mathbf{r}^{\prime}\right) A_{\mathrm{p}}\left(\mathbf{r}^{\prime}\right) e^{-j \Delta \mathbf{k} \cdot \mathbf{r}^{\prime}} d \mathbf{r}^{\prime} \\
& =\left.\frac{d_{\mathrm{eff}} \omega_{\mathrm{up}}^{2}}{c^{2}} \frac{e^{j k_{\mathrm{up}} r}}{2 \pi r} \mathcal{F}\left\{A_{\mathrm{IR}}\left(\mathbf{r}^{\prime}\right) A_{\mathrm{p}}\left(\mathbf{r}^{\prime}\right)\right\}\right|_{f=\frac{\Delta \mathbf{k}}{2 \pi}}
\end{aligned}
$$

Where $\mathcal{F}\{\}$ is the Fourier transform defined as $\left.\mathcal{F}\{g(\mathbf{r})\}\right|_{f}=\iiint_{-\infty}^{+\infty} g(\mathbf{r}) e^{-j 2 \pi f \cdot \mathbf{r}} d \mathbf{r}$ [68].

According to Eq. (12), the upconverted field in the Fraunhofer regime is proportional to the 3D Fourier transform of the spatial distribution of the source term. The Fourier transform is evaluated at the spatial frequencies $f=\Delta \mathbf{k} / 2 \pi$, where the phase mismatch term

$$
\Delta \mathbf{k}=\mathbf{k}_{\mathrm{up}}-\mathbf{k}_{\mathrm{IR}}-\mathbf{k}_{\mathrm{p}}
$$

is now a vectorial quantity. $\Delta \mathbf{k}$ is the phase mismatch between the generated upconverted field at an observation point and the polarization wave $S_{\text {up }}\left(\mathbf{r}^{\prime}\right)$ induced by the two incident fields (i.e., IR signal and pump).

From Eq. (12), we can derive an impulse response in the $k$-vector space by considering a plane wave IR signal as input, which corresponds to a unit-amplitude Dirac delta function in the $\mathbf{k}_{\mathrm{IR}}$-space (see Fig. 4). The impulse response function $h(\Delta \mathbf{k})$ is given by:

$$
h(\Delta \mathbf{k})=\left.\frac{2 d_{\mathrm{eff}} \omega_{\mathrm{up}}^{2}}{c^{2}} \mathcal{F}\left\{A_{\mathrm{p}}\left(\mathbf{r}^{\prime}\right)\right\}\right|_{f=\frac{\Delta \mathbf{k}}{2 \pi}},
$$

which is the upconverted field amplitude (i.e, output) produced in the $\Delta \mathbf{k}$-space. $h(\Delta \mathbf{k})$ describes the angular spread of multiple wavevectors $\mathbf{k}_{\text {up }}$ (all oscillating at $\omega_{\text {up }}$ ) that are generated from a plane wave IR signal defined by $\mathbf{k}_{\mathbf{I R}}$, as illustrated in a simplified model of the system (see Fig. 4).

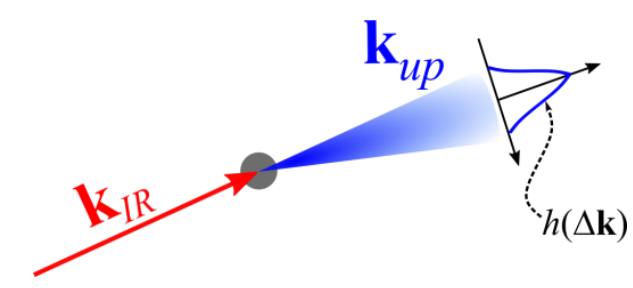

Fig. 4. An input IR plane wave or a single IR wavevector $\mathbf{k}_{\text {IR }}$ propagating inside the nonlinear medium (modeled as a point) and the corresponding output consisting of multiple upconverted wavevectors $\mathbf{k}_{\text {up }}$ with a common angular frequency $\omega_{\text {up. }}$. The impulse response function $h(\Delta \mathbf{k})$ describes the angular spread of wavevectors $\mathbf{k}_{\text {up. }}$

Equation (14) shows that the impulse response function is proportional to the 3-D Fourier transform of pump field spatial distribution bounded by the nonlinear medium. This is an easyto-remember piece of information describing the extent of the angular spread of the upconverted wavevectors as a result of a single IR input wavevector propagating inside the nonlinear medium. Strictly speaking, $h(\Delta \mathbf{k})$ is not $k$-vector space invariant. Even for a 
monochromatic IR signal and constant refractive index $n_{\mathrm{IR}}$, the profile of $h$ changes for different input $\mathbf{k}_{\mathrm{IR}}$ directions (or angles). Nevertheless, in cases where the $\mathbf{k}_{\mathrm{IR}}$ directions considered are close to the phase-matched direction, $h$ is appreciably invariant in this restricted $k$-space.

The important case of a focused Gaussian pump beam propagating along the $z$-axis has been treated analytically in [69] using Eq. (12). If diffraction of the Gaussian pump beam is ignored, the impulse response function reduces to:

$$
h=\frac{2 \pi w_{0}^{2} d_{\mathrm{eff}} \omega_{\mathrm{up} c}^{2} l_{c} A_{0, \mathrm{p}}}{c^{2}} e^{\frac{-\Delta k_{\mathrm{T}}^{2} w_{0}^{2}}{4}} \operatorname{sinc}\left(\frac{\Delta k_{z} l_{c}}{2}\right),
$$

where $\Delta k_{\mathrm{T}}^{2}=(\Delta \mathbf{k} \cdot \hat{\mathbf{x}})^{2}+(\Delta \mathbf{k} \cdot \hat{\mathbf{y}})^{2}$ and $\Delta k_{z}=\Delta \mathbf{k} \cdot \hat{\mathbf{z}}$. Assuming the input IR signal is a plane wave, $A_{0, \mathbb{R}} e^{j\left(\mathbf{k}_{\mathbb{R}} \cdot \mathrm{r}-\omega_{\mathbb{R}} t\right)}$, the upconverted field can be calculated by convoluting $h$ given by Eq. (15) and the Fourier transform of the IR signal as follows:

$$
\tilde{A}_{\text {up }}\left(\Delta k_{\mathrm{T}}, \Delta k_{z}\right)=\frac{2 \pi w_{0}^{2} d_{\mathrm{eff}} \omega_{\mathrm{up}}^{2} l_{c} A_{0, \mathrm{R}} A_{0, \mathrm{p}}}{c^{2}} e^{\frac{-\Delta k_{\mathrm{T}}^{2} w_{0}^{2}}{4}} \operatorname{sinc}\left(\frac{\Delta k_{z} l_{c}}{2}\right),
$$

where $A_{0, \mathrm{p}}$ and $A_{0, \mathrm{IR}}$ are the field amplitudes of the Gaussian pump field and the incident IR plane wave, respectively, $w_{0}$ is the beam radius $\left(1 / e^{2}\right)$ of the pump beam inside the nonlinear crystal. Equation (16) takes into account the length of the nonlinear crystal, $l_{c}$, and the crystal can therefore be thought of as a "thick filter" using terminology from Fourier optics.

From this expression, we can investigate the diffraction properties. We first note that the transverse part $\Delta k_{\mathrm{T}}$ of the phase mismatch vector enters the expression on equal terms as the axial component $\Delta k_{z}$, meaning that in a general theory, the phase mismatch vector, has no preferred direction evidently. In the vast relevant literature, the influence of $\Delta k_{\mathrm{T}}$ is ignored, i.e. $\Delta k_{\mathrm{T}}=0$. Using Eq. (16), we can provide the formal requirement here for $\Delta k_{\mathrm{T}}$. Firstly, a nonvanishing $\tilde{A}_{\text {up }}$ requires that $\left|\Delta k_{\mathrm{T}}\right| w_{0} \approx 0$. Secondly, if the pump beam radius $w_{0}$ is sufficiently large, $\tilde{A}_{\text {up }}$ is diminished unless $\Delta k_{\mathrm{T}}=0$. Strictly speaking the pump beam needs to be a plane wave, thus of infinite extent. Interestingly, in a linear medium, $\Delta k_{\mathrm{T}}=0$ leads to the classical Fresnel law of refraction, expressing the conservation of transverse momentum for a plane wave impinging on a medium of refractive index, $n$.

Equation (16) can be used to define the full-width-at-half-maximum (FWHM) of the upconverted intensity in the $k$-space for specific cases, however, it can be seen that if $\Delta k_{\mathrm{z}} l_{c} \ll\left|\Delta k_{\mathrm{T}}\right| w_{0}$, then the diffractive properties are determined predominantly by $\Delta k_{\mathrm{T}}$. This assumption corresponds to a thin filter approximation, well known from 2-D Fourier optics [68] and is usually fulfilled for upconversion imaging when $w_{0}$ is large. Note that $\Delta k_{z}$ is actively minimized through phase matching in birefringent crystals, in contrast to $\left|\Delta k_{\mathrm{T}}\right|$ (see section 3).

Assuming $\Delta k_{\mathrm{z}} l_{c} \ll\left|\Delta k_{\mathrm{T}}\right| w_{0}$, the resolution in $k$-space is determined from:

$$
\exp \left[\frac{-w_{0}^{2}\left|\Delta k_{\mathrm{T}, \mathrm{FWHM}}\right|^{2}}{8}\right]=\frac{1}{2} .
$$

From Eq. (17) we can derive 


$$
\left|\Delta k_{\mathrm{T}, \mathrm{FWHM}}\right|=\frac{2 \sqrt{2 \ln (2)}}{w_{0}} \text { or }\left|\Delta \psi_{\mathrm{FWHM}}\right|=\frac{\sqrt{2 \ln (2)} \lambda_{\text {up }}}{\pi w_{0}},
$$

where $\left|\Delta \psi_{\text {FWнм }}\right|$ is the angular spread of the upconverted field (external to the crystal). The resolution of an upconversion system is then determined by the pump beam waist wo which needs to be large to support high resolution imaging. Note that the crystal length, $l_{c}$, does not appear in Eq. (18). $l_{c}$ is present in the $\operatorname{sinc}\left(\Delta k_{z} l_{c} / 2\right)$ term as a modulation of the amplitude of the upconverted field. In linear imaging this modulation term is absent.

\subsection{Upconversion imaging: System-I}

Let us now consider an upconversion imaging system shown in Fig. 5 (also referred to as System-I). The first lens, $\mathrm{L}_{1}$, transforms the IR object field $g_{\mathrm{o}}(x, y)$ to the Fourier plane in the center of a nonlinear crystal. Inside the crystal, a Gaussian pump field interacts with the Fourier transformed IR object field through the second order nonlinearity of the crystal. The Fourier transformed IR object field can also be viewed as a superposition of plane waves, each of which has a complex amplitude given by $g_{0}(x, y)$. As described in sub-section 2.3, a single IR plane wave does not produce a single upconverted plane wave but rather a field with an angular spread in the form of Eq. (16). Again, assuming thin filter approximation (i.e., $\Delta k_{z} l_{c} \ll\left|\Delta k_{\mathrm{T}}\right| w_{0}$ ), the $k$-space angular spread of each output upconverted field due to each IR plane wave component is predominantly influenced by the term $\exp \left(-\Delta k_{\mathrm{T}}^{2} / 4\right)$ than by $\operatorname{sinc}\left(\Delta k_{z} l_{c} / 2\right)$. The second lens, $\mathrm{L}_{2}$, performs the final Fourier transform to bring the superposition of upconverted fields back to the spatial domain. Hence, the angular spread has a corresponding spatial spread in the image plane defined by the amplitude point-spread function (PSF).

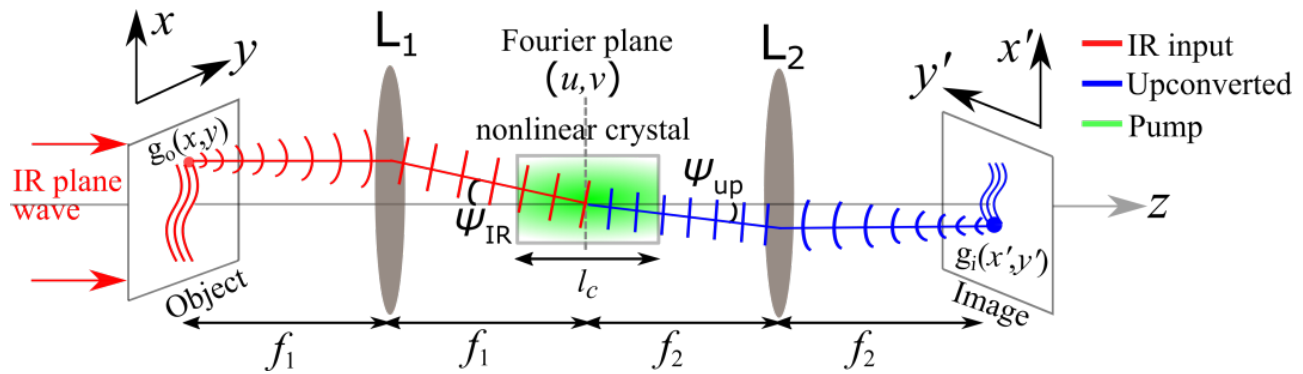

Fig. 5. System-I, upconversion takes place in a Fourier plane (transverse coordinates, $u, v)$. The object at one focal length $\left(f_{1}\right)$ in front of lens $\mathrm{L}_{1}$ is imaged at one focal length $\left(f_{2}\right)$ behind lens $\mathrm{L}_{2}$. An IR plane wave of uniform amplitude propagating along the $z$-axis illuminates the object transparency. The pump beam inside the crystal is indicated by green color. $g$ 's are the complex field distributions in the object and image planes. $\psi$ 's are the propagation angles (with $z$-axis) of IR and upconverted waves external to the crystal.

Given an IR signal field $g_{\mathrm{o}}(x, y)$ in the object plane, the upconverted field $g_{\mathrm{i}}\left(x^{\prime}, y^{\prime}\right)$ in the image plane of Fig. 5 is described by [70]:

$$
\begin{aligned}
& g_{\mathrm{i}}\left(x^{\prime}, y^{\prime}\right)=j \frac{2 \pi d_{\mathrm{eff}} l_{c} \lambda_{\mathrm{IR}} f_{1}}{n_{\mathrm{up}} \lambda_{\text {up }}^{2} f_{2}} A_{0, \mathrm{p}} \operatorname{sinc}\left[\Delta k_{\mathrm{z}}\left(\frac{x^{\prime}}{f_{2}}, \frac{y^{\prime}}{f_{2}}\right) \frac{l_{c}}{2}\right] \\
& \times g_{\mathrm{o}}\left(-\frac{\lambda_{\mathrm{IR}} f_{1}}{\lambda_{\text {up }} f_{2}} x^{\prime},-\frac{\lambda_{\mathrm{IR}} f_{1}}{\lambda_{\text {up }} f_{2}} y^{\prime}\right) \otimes\left(\frac{\pi w_{0}^{2}}{\lambda_{\mathrm{up}}^{2} f_{2}^{2}} \exp \left(-\pi^{2} w_{0}^{2}\left(x^{\prime 2}+y^{\prime 2}\right) /\left(\lambda_{\mathrm{up}} f_{2}\right)^{2}\right)\right),
\end{aligned}
$$


where $\varepsilon_{0}$ is the vacuum permeability, $E_{0, \mathrm{p}}$ is the maximum amplitude of the Gaussian pump with beam radius $w_{0}$, and $f_{1,2}$ are the focal lengths of lenses $\mathrm{L}_{1,2}$. In particular, the point-spread function (PSF) can be identified as $\left(\frac{\pi w_{0}^{2}}{\lambda_{\mathrm{up}}^{2} f_{2}^{2}} \exp \left(-\pi^{2} w_{0}^{2}\left(x^{\prime 2}+y^{\prime 2}\right) /\left(\lambda_{\mathrm{up}} f_{2}\right)^{2}\right)\right)$, which can accurately be simulated by the Fourier transform of a virtual "thin" Gaussian transmission filter in the center of the nonlinear crystal. Equation (19) can be decomposed into the following set of imaging actions:

(1) The object field is transferred to the image plane with a magnification $M=-\frac{\lambda_{\text {up }} f_{2}}{\lambda_{\mathrm{IR}} f_{1}}$.

(2) The resulting image generated from (1), is blurred by the PSF $\left(\frac{\pi w_{0}^{2}}{\lambda_{\text {up }}^{2} f_{2}^{2}} \exp \left(-\pi^{2} w_{0}^{2}\left(x^{\prime 2}+y^{\prime 2}\right) /\left(\lambda_{\text {up }} f_{2}\right)^{2}\right)\right)$, which is formed by the normalized 2-D optical Fourier transform of the Gaussian pump field profile, evaluated at spatial frequencies $x^{\prime} /\left(\lambda_{\text {up }} f_{2}\right)$ and $y^{\prime} /\left(\lambda_{\text {up }} f_{2}\right)[68]$.

(3) The sinc function, which acts like an amplitude mask, is superimposed on the imaging plane by multiplying the result in (2) by $\operatorname{sinc}\left(\Delta k_{z}\left(\frac{x^{\prime}}{f_{2}}, \frac{y^{\prime}}{f_{2}}\right) \frac{l_{c}}{2}\right)$, i.e., by propagating the angular sinc function to the image plane.

(4) The amplitude factor, $j \frac{2 \pi d_{\text {eff }} l_{c} \lambda_{\text {IR }} f_{1}}{n_{\text {up }} \lambda_{\text {up }}^{2} f_{2}} A_{0, \mathrm{p}}$, is multiplied to the image obtained in (3).

This four-step procedure constitutes a convenient and practical method for constructing the upconverted image. Furthermore, the FWHM of the square of the Gaussian PSF gives the size $d_{\text {res }}$ of resolvable image of a point object:

$$
d_{\mathrm{res}}=\frac{\sqrt{2 \ln 2} \lambda_{\mathrm{up}} f_{2}}{\pi w_{0}}
$$

From Eq. (18), we can also see that $d_{\text {res }}=\left|\psi_{\text {FWHM }}\right| f_{2}$.

\subsection{Upconversion imaging: System-II}

For completeness, we also consider upconversion in the image plane (System-II). Though Andrews [71], and later Milton [56], gave a detailed geometrical analysis of this configuration, we would like to briefly review the properties of System-II. To analyze the basic properties, we envision that the nonlinear crystal is situated in an intermediate image plane as shown in Fig. 6 , where the pump beam with radius $w_{0}$ illuminates the object's intermediate image. The intermediate image is a rescaled version of the object with a magnification factor $-z_{2} / z_{1}$ where $z_{1,2}>f_{1}$, which allows for matching the aperture size of the nonlinear crystal or the transverse size of the pump beam. Following a Huygens-Fresnel approach, consider a point source at point $\mathrm{P}$ in the intermediate image plane emitting a spherical upconverted wave. The need for phase matching effectively truncates (in angle) the emitted upconverted wave. The angular truncation 
is given by the acceptance angle, $\left|\psi_{\text {up,acc. }}^{\mathrm{FWHM}}\right|$, which is defined from points where the magnitude of the upconverted intensity is reduced to $50 \%$ of its maximum value. Essentially, $\left|\psi_{\text {up,acc. }}^{\mathrm{FWHM}}\right|$ limits the numerical aperture (N.A.) of the next lens $\mathrm{L}_{2}$ (see Fig. 6), thus the size $d_{\text {res }}$ of the resolvable image of the point source at $\mathrm{P}$ is then given by:

$$
d_{\text {res }} \approx \frac{2 \ln (2) \lambda_{\text {up }} z_{4}}{\pi\left|\psi_{\text {up,acc. }}^{\mathrm{FWHM}}\right| z_{3}} .
$$

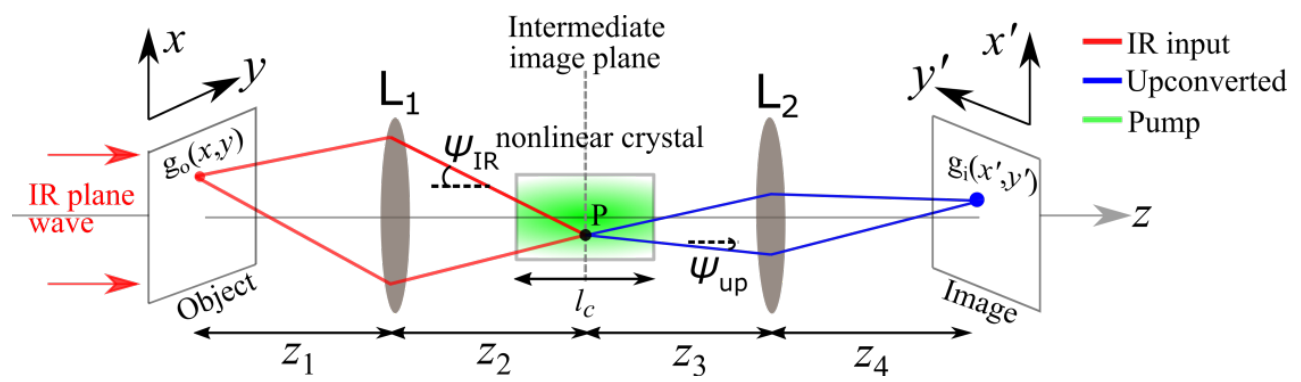

Fig. 6. System-II, upconversion takes place in an "intermediate" image plane. A point object $g_{0}(x, y)$ is first imaged by lens $\mathrm{L}_{1}$ of focal length $f_{1}$ at point $\mathrm{P}$ (inside the crystal) then the upconverted image $g_{i}\left(x^{\prime}, y^{\prime}\right)$ is formed by a second lens $\mathrm{L}_{2}$ of focal length $f_{2}$ in the final image plane. The $\mathrm{z}_{\mathrm{j}}$ 's are the object and image distances from the lenses.

It is interesting to note that for System-I, the pump beam diameter $w_{0}$, determines the spatial resolution for a given $\lambda_{\text {up }}$, but for System-II, it is $\left|\psi_{\text {up,acc. }}^{\mathrm{FWHM}}\right|$, thus a parameter determined by the phase matching properties and crystal length, $l_{\mathrm{c}}$. Since $\left|\psi_{\text {up,acc. }}^{\mathrm{FWHM}}\right|$ is often small (in the order of $0.01-0.1$ radian), $d_{\text {res }}$ for System-II is poor compared to the classical linear imaging case where the N.A. of the lens (e.g., 0.8 radian) results in better resolution.

System-I is more commonly used and has a few advantages over System-II in terms of robustness to crystal non-uniformity and the ability to perform spatial (Fourier) filtering by manipulating the pump beam profile. Henceforth, the following discussions in the article are based on System-I, unless otherwise specified. The imaging parameters of the two systems are compared in Table 2.

Table 2. Imaging parameters of System-I and System-II

\begin{tabular}{|c|c|c|}
\hline Parameter & System-I & System-II \\
\hline FoV & Phase matching condition, $\sim \operatorname{sinc}^{2}\left(\frac{\Delta k_{\mathrm{z}} l_{c}}{2}\right)$ & $\begin{array}{l}\text { Pump diameter }\left(2 w_{0}\right) \text { and/or crystal aperture } \\
\text { size, whichever is smaller }\end{array}$ \\
\hline Resolution & $\begin{array}{l}\text { Size of resolvable element in the image plane, } \\
\qquad d_{\text {res }}=\frac{\sqrt{2 \ln (2)} \lambda_{\text {up }} f_{2}}{\pi w_{0}}\end{array}$ & 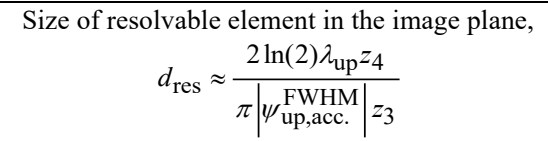 \\
\hline Magnification & $M=-\frac{f_{2} \cdot \lambda_{\text {up }}}{f_{1} \cdot \lambda_{\mathrm{IR}}}$ & $M=\frac{z_{2} \cdot z_{4}}{z_{1} \cdot z_{3}}, z_{1,2}>f_{1}$ and $z_{3,4}>f_{2}$ \\
\hline Efficiency & $\begin{array}{l}\text { For Gaussian pump and within the FoV, the } \\
\text { efficiency decreases for higher spatial } \\
\text { frequencies. }\end{array}$ & $\begin{array}{l}\text { For Gaussian pump and within the FoV, the } \\
\text { efficiency decreases for off-axis object points. }\end{array}$ \\
\hline
\end{tabular}




\section{Phase matching (PM)}

The term phase matching (PM) has been introduced in the description of parametric frequency upconversion in section 2. In this section, the fundamental concept of PM is discussed in more detail, first considering PM of collinear plane waves, followed by a description of PM of noncollinear waves, which is relevant for upconversion imaging. From a physical point of view, PM means that the phase velocity of the induced second order polarization (originating from the input pump and IR signal) should match the phase velocity of the generated field (upconverted); this results in constructive addition of the generated fields from the induced dipoles along the direction of propagation. Collinear interaction can be along one of the principal axes of the material, called non-critical phase matching (NCPM), or in a direction away from the crystallographic axis, generally termed critical phase matching (CPM). For upconversion imaging, the interacting fields are generally non-collinear. For the non-collinear case, the PM condition has to be fulfilled, both for the longitudinal and the transverse part of the wavevectors. PM is important in order to maximize the conversion efficiency; however, even for non-perfect PM, some conversion still occurs. In sub-section 3.3, the acceptance parameters are considered, i.e. how fast the conversion efficiency decreases as a function of different parameters, e.g. the propagation angle through the material, and how different parameters can be used to control the PM wavelengths. Note that all angles $(\theta, \phi)$ described in this section are propagation angles inside the nonlinear crystal. The external angles can be calculated using Snell's law with the corresponding refractive index of the material.

\subsection{Collinear phase matching}

Considering parametric exchange of energy between three monochromatic, continuous, plane waves propagating through a nonlinear crystal, both energy, Eq. (2), and momentum, Eq. (13), have to be conserved. The energy is strictly conserved for parametric processes, as no energy is exchanged with the material. The momentum conservation accounts for the coherent addition of the radiated fields from the induced dipoles. As the momentum conservation describes the relative phase of the induced dipoles and the propagating optical field at the same frequency, this relation is often termed as the PM condition.

In nonlinear materials used for parametric frequency conversion, the refractive index depends not only on the wavelength (dispersion), but also on the temperature of the nonlinear material, the polarization of the interacting fields, and their angle of propagation relative to the crystallographic axis of the material. Thus, the PM condition can be expressed as

$$
\frac{n_{\mathrm{p}}\left(\theta, T, \lambda_{\mathrm{p}}\right)}{\lambda_{\mathrm{p}}}+\frac{n_{\mathrm{IR}}\left(\theta, T, \lambda_{\mathrm{RR}}\right)}{\lambda_{\mathrm{IR}}}=\frac{n_{\mathrm{up}}\left(\theta, T, \lambda_{\mathrm{up}}\right)}{\lambda_{\mathrm{up}}},
$$

where the refractive index is calculated as a function of angle, temperature and wavelength.

Due to normal material dispersion, i.e. decreasing refractive index for longer wavelengths, Eq. (22) has no solution if all three waves are propagating collinearly with parallel linear states of polarization in a homogeneous bulk material. A common approach to obtain PM is to exploit the anisotropy of the nonlinear material, i.e. exploit interaction of fields of orthogonal linear polarization to compensate for the dispersion induced phase mismatch - this is known as birefringent PM (BPM). In 1962, Giordmaine [34] and Maker et al. [35] independently proposed and demonstrated BPM based $\chi^{(2)}$ interaction. Two configurations can be used for upconversion: (i) Type-I PM where the two inputs (pump and IR signal) are linearly polarized in the same direction and an upconverted field is generated in the orthogonal polarization state, or (ii) Type-II PM where the two inputs have orthogonal, linearly polarized states.

For anisotropic materials, the refractive indices are generally described by a $3 \times 3$ tensor. In a unique orthogonal coordinate system, known as principal axes $(\hat{a}, \hat{b}, \hat{c})$, the tensor 
becomes diagonal, where the diagonal elements are known as principal refractive indices ( $\left.n_{\hat{a}}, n_{\hat{b}}, n_{\hat{c}}\right)$ [61]. A crystal for which $n_{\hat{a}} \neq n_{\hat{b}} \neq n_{\hat{c}}$ is known as biaxial, whereas, if two of the principal indices are identical, i.e. $n_{\hat{a}}=n_{\hat{b}} \neq n_{\hat{c}}$, the crystal is called uniaxial. In uniaxial crystals the crystallographic $\hat{c}$-axis is known as the optic axis. The following description is focused on uniaxial crystals; however, it is straightforward to expand the treatment to biaxial crystals, particularly considering operation in a principal plane of the crystal.

In a uniaxial crystal, light polarized perpendicular to the plane containing its propagation wavevector $\mathbf{k}$ and the optic axis $\hat{c}$ is known as the ordinary ray or $o$-ray and the corresponding refractive index is defined as $n_{o}=n_{\hat{a}}=n_{\hat{b}}$. The field polarized in the plane containing $\mathbf{k}$ and $\hat{c}$ is known as the extraordinary ray or $e$-ray and the associated refractive index is $n_{e}(\theta)$, which is a function of the propagation direction, i.e. the angle $\theta$ between $\mathbf{k}$ and $\hat{c}$ as follows

$$
\frac{1}{n_{e}^{2}\left(\theta_{\mathrm{j}}, \lambda_{\mathrm{j}}, T\right)}=\frac{\sin ^{2} \theta_{\mathrm{j}}}{n_{e}^{2}\left(\theta_{\mathrm{j}}=90^{\circ}, \lambda_{\mathrm{j}}, T\right)}+\frac{\cos ^{2} \theta_{\mathrm{j}}}{n_{o}^{2}\left(\lambda_{\mathrm{j}}, T\right)} \quad \text {, where } \mathrm{j}=\mathrm{p}, \mathrm{IR} \text {, up. }
$$

Typically, the highest conversion efficiency is obtained for collinear interaction, i.e. all fields propagating in the same direction, resulting in the best possible spatial overlap of the interacting fields, this is the configuration of choice for most parametric (non-imaging) frequency conversion devices. If the propagation direction is along one of the principal axes of the material, it is generally called NCPM, whereas, if the fields propagate non-parallel to the crystallographic axis, it is called CPM as discussed in the following paragraphs.

\section{Non-critical phase matching (NCPM):}

In some materials, the PM condition Eq. (22) can be fulfilled for interaction of plane waves propagating along one of the principal axes of the material, by appropriate choice of the direction of polarization of the two co-propagating input fields and the temperature of the nonlinear material, see Fig 7. In this configuration, the temperature of the material is used to optimize the parametric interaction for a specific combination of wavelengths to fulfill the PM condition. The resulting upconverted field propagates in the same direction as the two input waves, and, when phase matched, the generated field builds up constructively as it propagates trough the material as described in section 2. Temperature tuned NCPM has been demonstrated in several conversion devices [18], [72].

The interaction of co-propagating fields along a principal axis of the nonlinear material is particularly advantageous, as beam walk-off is absent - a desirable configuration described in section 2. This ensures optimal spatial overlap and interaction length for the parametric upconversion process. Furthermore, the first order derivative of the phase mismatch term with respect to a tuning parameter is zero, making this configuration less sensitive to parameter variation, hence the term non-critical phase matching, which is discussed in more detail in subsection 3.3.
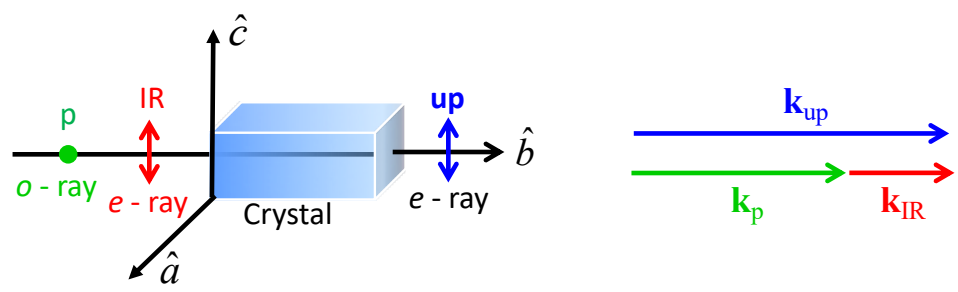

Fig. 7. Illustration of NCPM Type-II upconversion. The input fields are orthogonally polarized and propagating collinearly, along one of the principal axes of the nonlinear material. 


\section{Quasi phase matching (QPM):}

It is not always possible to obtain PM for two input waves co-propagating along a principal axis of the nonlinear material, using only the temperature as tuning parameter. If the nonlinear crystal does not possess sufficient thermal birefringence and anisotropy to compensate for the dispersion induced phase mismatch, a different PM technique called quasi PM (QPM) can be implemented. In this approach, the phase mismatch $(\Delta \mathbf{k})$ can be compensated using a spatially structured nonlinear material. Due to the non-centrosymmetric nature of $\chi^{(2)}$ materials, an inversion of the crystal structure results in a change of the sign of the nonlinearity, i.e. introducing a $\pi$-phase shift. Using a periodic structure, in which consecutive sections of the nonlinear material have inverted domains as seen in Fig 8(a), it is possible to periodically compensate for the accumulated phase mismatch between the induced polarization and the upconverted signal propagating through the material.
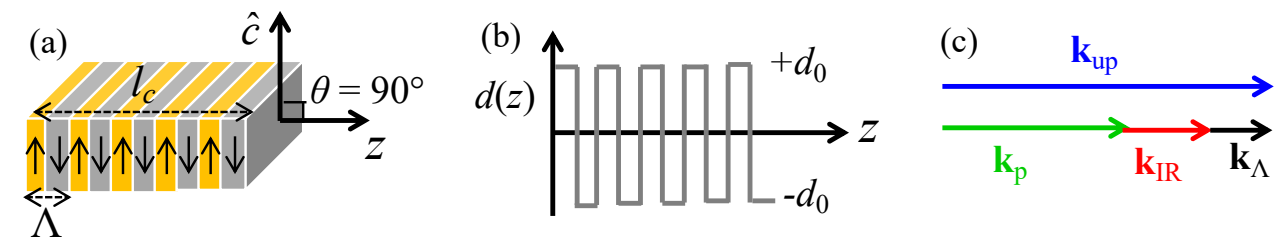

Fig. 8. Schematic of a (a) periodically poled crystal for QPM. The angle between crystal $\hat{c}$-axis
and laboratory $z$-axis/ $\mathbf{k}_{\mathrm{p}}$ direction is $\theta$, which is $90^{\circ}$ in case of QPM crystal. The solid arrows are
indicating the $\hat{c}$-axis orientation of each poled section. The poling periodicity is $\Lambda$. (b) Variation
of nonlinear coefficient, $d$ along the length of the poled crystal. (c) Collinear PM diagram of a
QPM condition, where $\left|\mathbf{k}_{\Lambda}\right|=2 \pi / \Lambda$.

The periodic modulation of the material susceptibility seen in Fig. 8(b) can be treated as a square wave in the material, consequently, it can be expanded in all odd harmonic frequencies of its periodicity. Its Fourier space wavevectors can be easily found as given in Eq. (24), where $m$ denotes the harmonic order and $\Lambda$ is the spatial period. Furthermore, as it is a standing wave, it is the superposition of a forward and a backward traveling wave; hence, the sign of the wavevector can be either positive or negative.

$$
k_{\Lambda}^{m}=\frac{2 \pi m}{\Lambda}, \quad \text { where } m= \pm 1, \pm 3, \pm 5, \ldots
$$

Using this approach, the new PM condition is given by Eq. (25), with a designable parameter $\Lambda$ to obtain PM, without relying solely on the anisotropy and thermal dispersion parameter of the material (see Fig. 8(c)).

$$
\mathbf{k}_{\mathrm{up}}-\mathbf{k}_{\mathrm{p}}-\mathbf{k}_{\mathrm{IR}}-\frac{2 \pi m}{\Lambda}=0
$$

This approach further allows the freedom to choose the field polarizations that give access to the largest nonlinear susceptibility tensor element of the material. Generally, when all interaction waves are linearly polarized along the optic axis of the periodically poled crystal, the largest second order coefficient, $d_{33}$, is accessed. Hence, higher efficiency for the frequency conversion is achievable, even though it is reduced by the amplitude of the harmonic Fourier component of the induced grating,

$$
d_{\text {eff }}=d_{33} G_{m}, \quad G_{m}=\frac{2}{\pi m}, \quad m=1,3,5, \ldots
$$


The $1^{\text {st }}$ order structure is the preferred choice for QPM based frequency conversion, as seen from Eq. (26). According to the specifications provided by one of the nonlinear crystal supplier [73], a $5 \% \mathrm{MgO}$-doped $\mathrm{LiNbO}_{3}$ at $1064 \mathrm{~nm}$ yields $d_{31}=4.4 \mathrm{pm} / \mathrm{V}$ (typically used for BPM) and $d_{33}=25 \mathrm{pm} / \mathrm{V}$ (used for QPM). Thus, for the $1^{\text {st }}$ order QPM, $d_{\text {eff }} \sim 14 \mathrm{pm} / \mathrm{V}$, which is more than three times the value of $d_{31}$, i.e. about 10 times higher conversion efficiency (according to Eq. (10)) compared to BPM in the same material (provided that all other parameters remain unchanged).

From a historical perspective, QPM was first suggested by Armstrong in 1962 [12] and mentioned by Franken and Ward in 1963 [74], however, the fabrication process made it difficult to realize QPM structures experimentally. During the late 1970's, stacked plates based QPM crystals were developed [38], [39], however, the periodicity was limited to several 100's of $\mu \mathrm{m}$ using crystal plates of transverse dimension $9 \mathrm{~mm} \times 28 \mathrm{~mm}$ [39]. Later, epitaxial growth based technique, as well as diffusion bonded crystals, were developed enabling shorter periods and resulting in more versatile performance [75], [76]. Nowadays, domain reversal techniques are commonly used, which is based on the application of a high external electric field across the ferroelectric crystals, providing reliable poling periods ranging from few $\mu \mathrm{m}$ to 10 's of $\mu \mathrm{m}$ for crystal thickness of $0.1-2 \mathrm{~mm}$ [73], [77], [78]. An alternative approach is molecular beam epitaxy applied for semiconductor crystals, producing grating periods ranging from 10s to $100 \mathrm{~s}$ of $\mu \mathrm{m}$. Structures of $20.8 \mu \mathrm{m}$ grating period have been reported for a GaP crystal with thickness of $150 \mu \mathrm{m}$ and $61 \mu \mathrm{m}$ grating period for $0.5 \mathrm{~mm}$ thick GaAs crystal [79], [80].

From an operational point of view, QPM can be considered as a special case of NCPM, as the interacting fields are all propagating along one of the principal axes of the nonlinear crystal. The highest upconversion efficiencies has been obtained with QPM devices [81], [82]. These devices are suitable for single-photon counting [81], [83]-[86], spectroscopy [87]-[89], and imaging [45], [90]-[95]. The transverse dimension of standard QPM crystals is typically limited to approximately $1 \mathrm{~mm}$ for periodically poled lithium niobate (PPLN) [73], [87]. This imposes a limitation on the imaging FoV (particularly for long PPLN crystals) as well as on the spatial resolution due to the limited crystal aperture size (which sets an upper limit to $w_{0}$ ), as seen in Table 2. However, recent technological development results in PPLN of larger thickness [78], which will definitely improve the imaging quality. On the other hand, QPM configuration has also been successfully employed for raster-scan based upconversion imaging [91], [96].

\section{Critical phase matching (CPM):}

In some cases, it is not possible to obtain PM for two collinearly propagating input waves based on the anisotropy of the material and control of the material temperature, when the fields are propagating along the crystallographic axis of the material. Choosing a propagation direction non-parallel to the principal axis of the material, can enable PM, as illustrated in see Fig. 9. However, because of the anisotropy of the material, walk-off occurs for the extraordinary polarized fields, limiting the interaction length of the parametric process (see Fig. 9(c)). The walk-off angle increases for larger angles relative to the nearest crystallographic axis. The angle is typically in the order of few degrees and it attains a maximum value for $\theta=45^{\circ}$ [64], [67]. Using CPM, Type-I interaction is preferred over Type-II, as for Type-I the two inputs are of the same polarization, hence, even if they walk-off (extraordinary polarized), they do so in a similar direction and angle [97], maintaining spatial overlap for longer interaction length, in comparison to Type-II. 

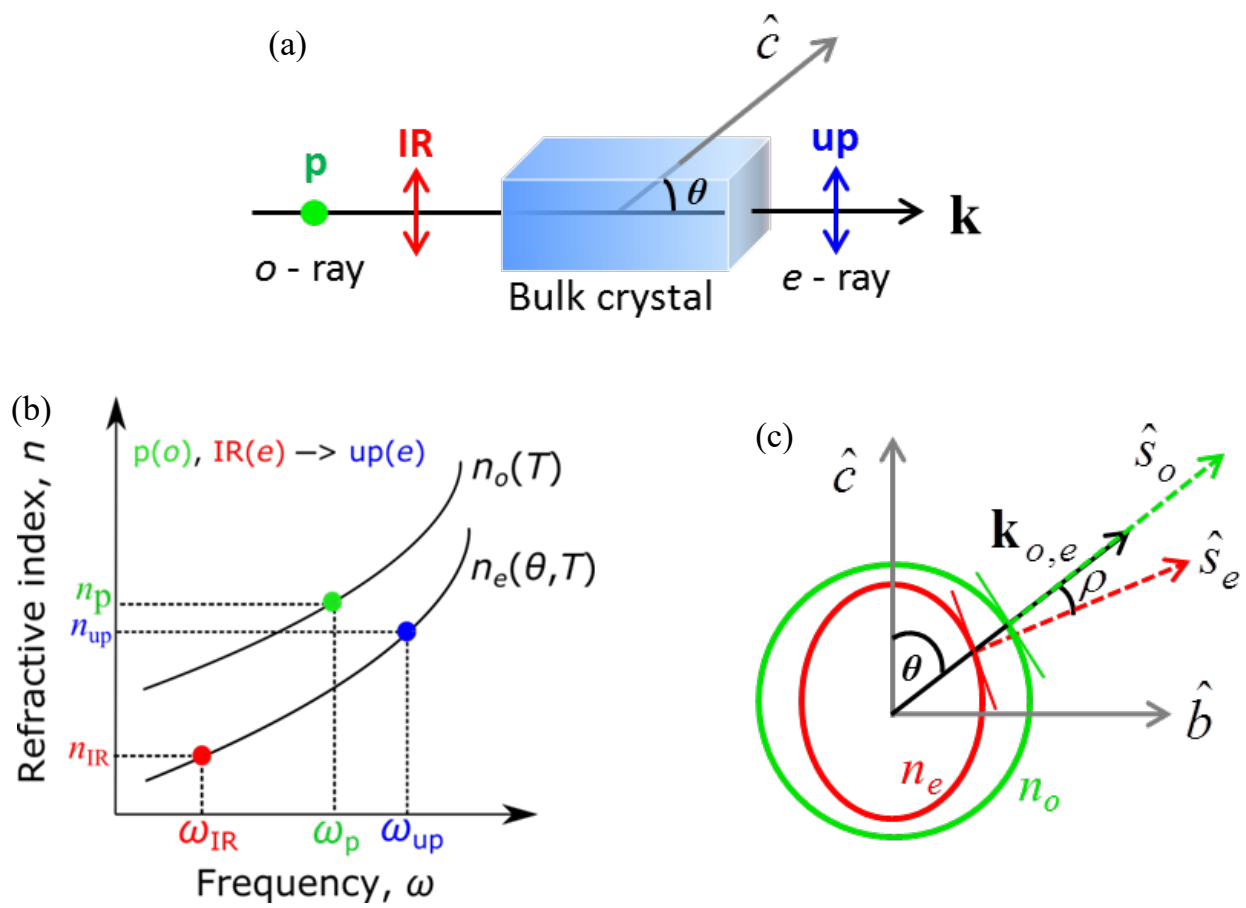

Fig. 9. (a) Concept of $o$-ray and $e$-ray. The wavevector, $\mathbf{k}$ makes angle $\theta$ to the crystal $\hat{c}$-axis. (b) Concept of temperature $(T)$ and angle $(\theta)$ dependent birefringent phase matching. (c) Schematic representation of index ellipsoid projected on crystal $c-b$ plane; and the angle-dependent refractive index resulting in a spatial walk-off between the $o$-ray and the $e$-ray even when they propagate/phase match collinearly.

\subsection{Non-collinear phase matching}

In sub-section 3.1, collinear propagation of three waves has been considered. In this subsection, the analysis is extended to include non-collinear propagating plane waves. Whereas the PM condition in the previous section was simplified to consider only the length of the wavevectors (as they are all parallel), non-collinear interaction requires a vectorial analysis. Defining the direction of the pump wave as the $z$-direction (longitudinal direction), the PM condition has to be fulfilled in both the longitudinal and the transverse direction. The condition can be divided into two scalar equations Eq. (27) and Eq. (28), where $\phi_{\mathrm{j}}$ is the angle of the $\mathrm{j}^{\text {th }}$ wave relative to the pump direction inside the nonlinear material, and $\theta_{\mathrm{j}}$ is the angle of the wave relative to the $\hat{c}$-axis of the material (considering a uniaxial material).

$$
\begin{gathered}
\Delta k_{\mathrm{z}}=\left|\mathbf{k}_{\text {up }}\left(\theta_{\text {up }}\right)\right| \cos \left(\phi_{\text {up }}\right)-\left|\mathbf{k}_{\mathrm{p}}\left(\theta_{\mathrm{p}}\right)\right|-\left|\mathbf{k}_{\mathrm{IR}}\left(\theta_{\mathrm{IR}}\right)\right| \cos \left(\phi_{\mathrm{IR}}\right) \\
\Delta k_{\mathrm{T}}=\left|\mathbf{k}_{\text {up }}\left(\theta_{\text {up }}\right)\right| \sin \left(\phi_{\text {up }}\right)-\left|\mathbf{k}_{\mathrm{IR}}\left(\theta_{\mathrm{IR}}\right)\right| \sin \left(\phi_{\mathrm{IR}}\right)
\end{gathered}
$$

where $\Delta k_{\mathrm{z}}$ and $\Delta k_{\mathrm{T}}$ are the longitudinal and transverse phase mismatch, respectively. Using these equations, the non-collinear PM conditions can be calculated for both Type-I and TypeII interactions as well as for QPM structures adding an additional term for the wavevector of the induced structure $\mathbf{k}_{\Lambda}$ in Eq. (27). Considering plane wave interaction, the transverse equation must be strictly fulfilled, i.e. $\Delta k_{\mathrm{T}}=0$, resulting in image transformation from the input to the output side, however, with a magnification factor proportional to the wavelength ratio $\lambda_{\text {up }} / \lambda_{\text {IR }}$, as shown in Table 2 . 
Assuming a uniaxial nonlinear material with a pump wave propagating along one of the principal planes, the angular dependent refractive indices can be easily calculated along the lines outlined in [98], using the notation presented in Fig. 10.

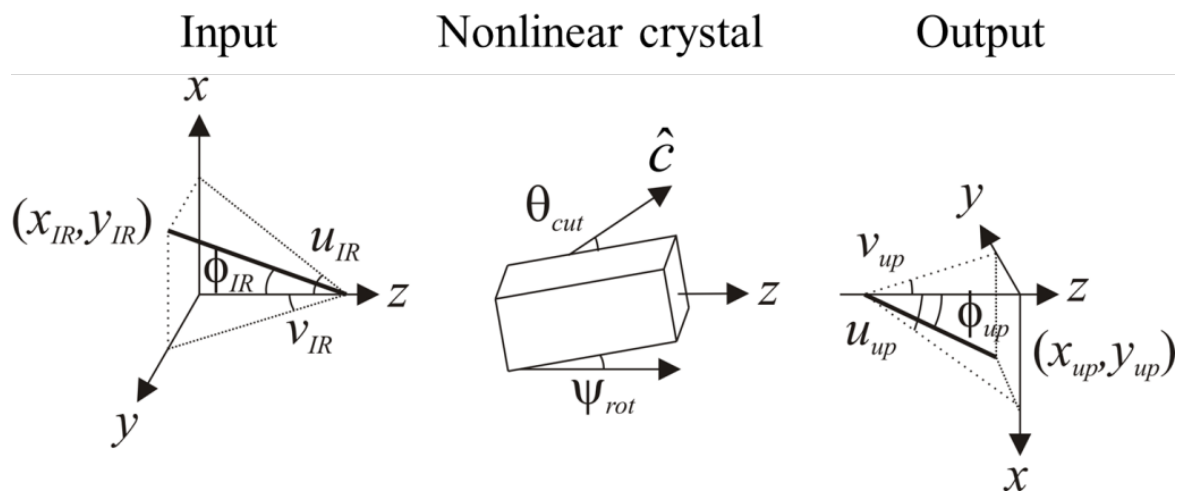

Fig. 10. Schematic representation of coordinate transformation scheme for calculating noncollinear phase matching conditions. The $x-y-z$ is laboratory coordinate system, where the pump is along the $z$-axis. The angle $u_{\mathrm{j}}$ and $v_{\mathrm{j}}$ are projected in the $x-z$ and $y-z$ plane, respectively. $\phi$ and $\psi$ are internal and external angles (with respect to the crystal), respectively. All notations are the same as described in above text.

The angels $\left(\theta_{\mathrm{j}}, \phi_{\mathrm{j}}\right)$ are easily calculated using Eq. (29) and Eq. (30), as a function of the input angles of the signal wave and the output angles of the upconverted wave.

$$
\begin{gathered}
\cos \left(\phi_{\mathrm{j}}\right)=\frac{1}{\sqrt{1+\tan ^{2}\left(u_{\mathrm{j}}\right)+\tan ^{2}\left(v_{\mathrm{j}}\right)}} \\
\cos \left(\theta_{\mathrm{j}}\right)=\frac{\cos \left(\theta_{\text {cut }}+u_{\mathrm{j}}+\psi_{\text {rot }}\right) \cos \left(v_{\mathrm{j}}\right)}{\sqrt{\cos ^{2}\left(v_{\mathrm{j}}\right)+\cos ^{2}\left(u_{\mathrm{j}}\right) \sin ^{2}\left(v_{\mathrm{j}}\right)}} .
\end{gathered}
$$

The angles $u_{\mathrm{j}}$ (within the principle plane) and $v_{\mathrm{j}}$ (orthogonal to the principle plane) describe the internal angles of the input IR and generated up wave. $\theta_{\text {cut }}$ and $\psi_{\text {rot }}$ are the cut angle of the nonlinear crystal relative to the $\hat{c}$-axis and the rotation angle of the crystal relative to normal incidence, respectively. Having calculated $\theta_{\mathrm{j}}$ and $\phi_{\mathrm{j}}$, and the polarization direction of the waves, the refractive indices and hence the wavevectors can be inserted into Eq. (27) and Eq. (28). If $\Delta k_{\mathrm{z}}=0$ and $\Delta k_{\mathrm{T}}=0$, a plot of the PM upconverted wavelengths and the corresponding angles of the upconverted wave can be obtained. This is discussed further in the following sub-section.

\subsection{Acceptance parameters and phase match tuning}

The different PM schemes are summarized in Fig. 11. The two axis of the coordinate system describes the two principal axes of the nonlinear material. For the ordinary polarized wave, the refractive index is independent of the direction of propagation, i.e. the refractive index forms a circle, whereas Eq. (23) describes the refractive index of an extraordinary polarized wave, i.e. forming an ellipse with its major and minor axes along two principal axes of the nonlinear material. From Fig. 11 it is clear, that if the input fields are propagating collinearly along one of the principal axes, the circle and the ellipse intersect tangentially, these two directions correspond to the NCPM as mentioned in sub-section 3.1. For the direction marked with A, two collinear beams are phase matched, however, the ellipse and the circle describing the refractive indices are intersecting rather being tangential, corresponding to CPM. All other combinations leading to PM rely on non-collinear PM. In addition to the propagation along on of the principal 
axis, one additional point, marked with $\mathrm{B}$, leads to tangential alignment of the refractive indices, this special form of non-collinear PM is known as tangential PM (TPM) [22].
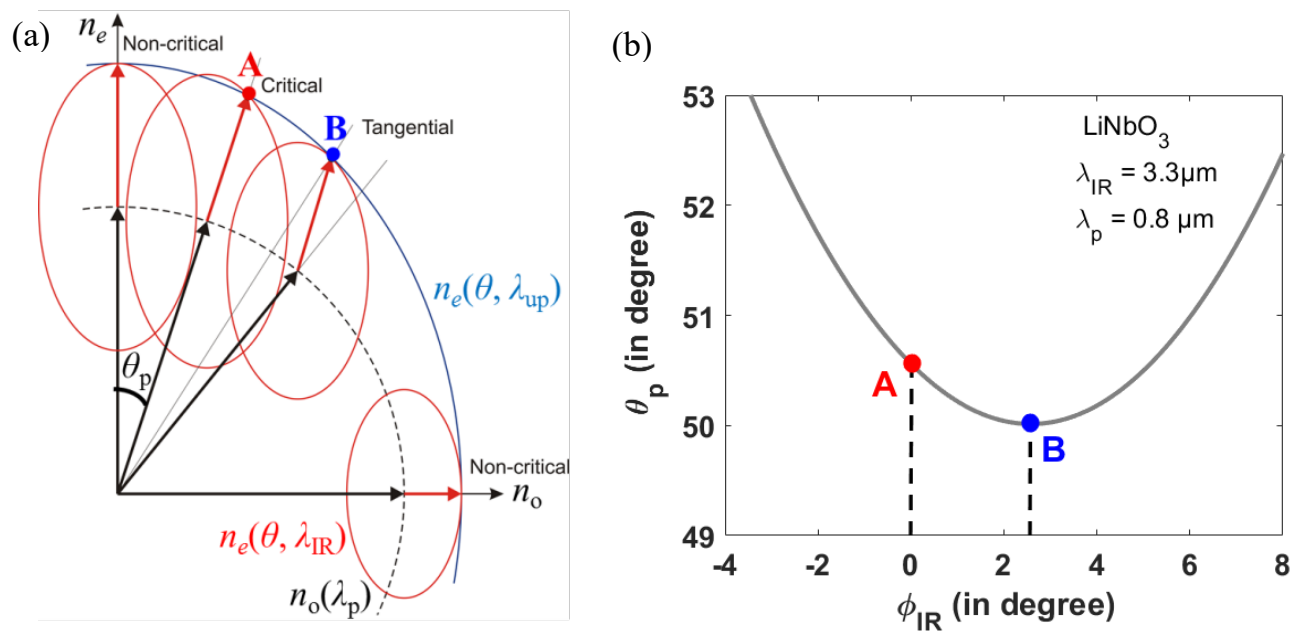

Fig. 11. (a) Different types of BPM in a negative uniaxial crystal. The symbols "o" and "e" represent $o$-ray and $e$-ray, respectively. Other symbols are described previously in text. The black and red solid arrows indicate pump and IR wavevector, respectively. (b) BPM in a $\mathrm{LiNbO}_{3}$ crystal calculated for pump and infrared wavelength of $0.8 \mu \mathrm{m}$ and $3.3 \mu \mathrm{m}$, respectively. The point $\mathrm{A}$ and $\mathrm{B}$ indicate collinear CPM and non-collinear tangential phase matching, respectively. Both the points (A, B) correspond to (a).

As seen in sub-sections 3.1 and 3.2, PM can be achieved for a range of configurations, using parameters like angle, wavelength, and temperature. If the PM condition is not perfectly fulfilled, it was discussed in section 2, that the conversion efficiency, in a lossless medium, is proportional to $\operatorname{sinc}^{2}\left(\Delta k_{z} l_{c} / 2\right)$. This shows, that a non-zero phase mismatch, $\Delta k_{z}$, calculated along the pump direction, reduces the conversion efficiency. The tolerance on the PM parameters is described by their acceptance parameters, referred to as: (i) angular acceptance bandwidth $\left(\Delta \theta_{\mathrm{j}}\right)$, (ii) spectral acceptance bandwidth $\left(\Delta \lambda_{\mathrm{j}}\right)$, and (iii) temperature acceptance bandwidth $(\Delta T)$, respectively. The acceptance parameter is generally defined as FWHM values. Each acceptance parameter can be calculated or measured while keeping all other parameters constant.

The acceptance parameters are typically evaluated from the variation in $\Delta k_{\mathrm{z}}$, assuming $\Delta k_{\mathrm{T}}=0$. Due to this "sinc-squared" nature of the upconversion efficiency, it decreases as $\left|\Delta k_{z} l_{c} / 2\right|$ increases, such that it reaches a maximum value when $\Delta k_{\mathrm{z}}=0$ and becomes zero for $\Delta k_{\mathrm{z}}=2 \pi / l_{c}$. As $\left|\Delta k_{\mathrm{z}}\right|$ is further increased, the efficiency shows oscillatory behavior with sharply decreasing amplitude. While the energy conservation, i.e. Eq. (2), is strictly satisfied for parametric frequency conversion, the efficiency of the process follows the $\operatorname{sinc}^{2}$ function for the wavevector mismatch for a finite crystal length. The efficiency remains high for small phase mismatch $\Delta k_{\mathrm{z}}<<2 \pi / l_{c}$ and therefore a small deviation from the exact PM can be tolerated. In the case of a shorter crystal length, the acceptance bandwidth increases, however, with a penalty in efficiency. Using a first order derivative, the dependence of $\Delta k_{\mathrm{z}}$ on the PM parameters can be approximated as

$$
\begin{aligned}
\Delta k_{z}\left(\theta_{\mathrm{j}}, \lambda_{\mathrm{j}}, T\right) & \left.\simeq \Delta k_{z}\right|_{\mathrm{PM}}(=0)+\delta\left(\Delta k_{z}\right) \\
& =\frac{\partial\left(\Delta k_{z}\right)}{\partial \theta_{\mathrm{j}}} \Delta \theta_{\mathrm{j}}+\frac{\partial\left(\Delta k_{z}\right)}{\partial \lambda_{\mathrm{j}}} \Delta \lambda_{\mathrm{j}}+\frac{\partial\left(\Delta k_{z}\right)}{\partial T} \Delta T
\end{aligned}
$$


For a monochromatic pump propagating along a certain direction, the acceptance parameters can be calculated in terms of IR wave parameters $\left(\Delta \theta_{\mathrm{IR}}, \Delta \lambda_{\mathrm{IR}}, \Delta T\right)$ for different types of PM conditions [28], [99]. In case of NCPM and TPM, the $d\left(\Delta k_{z}\right) / d \theta=0$ for a perfect PM point, which essentially provides wider acceptance parameters in comparison to the CPM condition [22]. However, in that case, the second order derivative must be taken into account when calculating the acceptance parameters. Typically the angular acceptance bandwidth is in the order of a few 10's of mrad (CPM) [18], [100] to a few 100's of mrad (NCPM/TPM) for the IR wave [22]; correspondingly the spectral acceptance bandwidth varies from a few nm to a few 10's of nm [18], [22], [100]-[102]. The temperature acceptance bandwidth is typically in the order of a few ${ }^{\circ} \mathrm{C}$ [72], [102], [103]. These are important parameters when studying multispectral imaging and FoV of the images. For broadband or hyperspectral imaging, a wider range of $\lambda_{\text {IR }}$ needs to be upconverted simultaneously, which can be achieved by considering, e.g. non-collinear interaction [87], temperature tuning [45], [88], rotating the crystal [104], tuning the pump wavelength [93], and/or using a broadband pump source [95].

Returning to Fig. 11, it is clear that a small change of angle will have a symmetric impact on the PM condition, when the interacting fields are propagating along one of the principal axes of the material. This means, that a phase matched upconversion process at a non-collinear angle $\phi_{\text {IR }}$ relative to the pump, results in an upconverted field at angle $\phi_{\text {up }}$ symmetrically around the pump beam, as seen in Fig. 12(a). Point B refers to the collinear NCPM point collinear with the principal axis of the nonlinear material, and this is also the case for QPM as indicated in the figure. Considering longer IR wavelengths leads to PM angles as indicated in Fig. 12(b). Thus, non-collinear interaction increases the spectral range of upconversion as well as the FoV for imaging [45], [87], [98], assuming that the IR signal is not monochromatic.
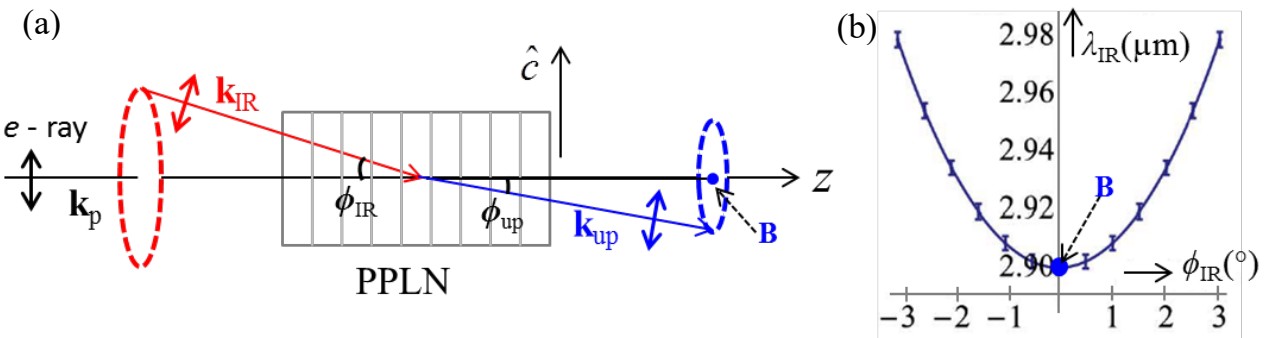

Fig. 12. Non-collinear upconversion in a periodically poled crystal, (a) wavevector directions and PM ring pattern in a PPLN crystal, (b) phase matching curve for a PPLN with poling period $=21 \mu \mathrm{m}$ and $\lambda_{\mathrm{p}}=1064 \mathrm{~nm}$. The $\theta_{\mathrm{p}}$ is $90^{\circ}$, which makes the TPM point (B) on axis, indicated by dashed arrow. The error bar in (b) indicates the spectral acceptance bandwidth.

For the case of CPM, the symmetry point B of the upconverted wavelengths is no longer coincident with the pump beam axis (i.e. $z$-axis), but displaced at an angle corresponding to the TPM direction, as seen in Fig. 13. This understanding is very important for the image formation considered in the following sections.

The angular acceptance parameter further implies that the width of the upconverted ring, for a monochromatic IR input, is proportional to the angular acceptance parameter, meaning that the upconverted ring is broader close to the NCPM configuration, but narrower further away. This feature is considered further in sub-section 5.4. 


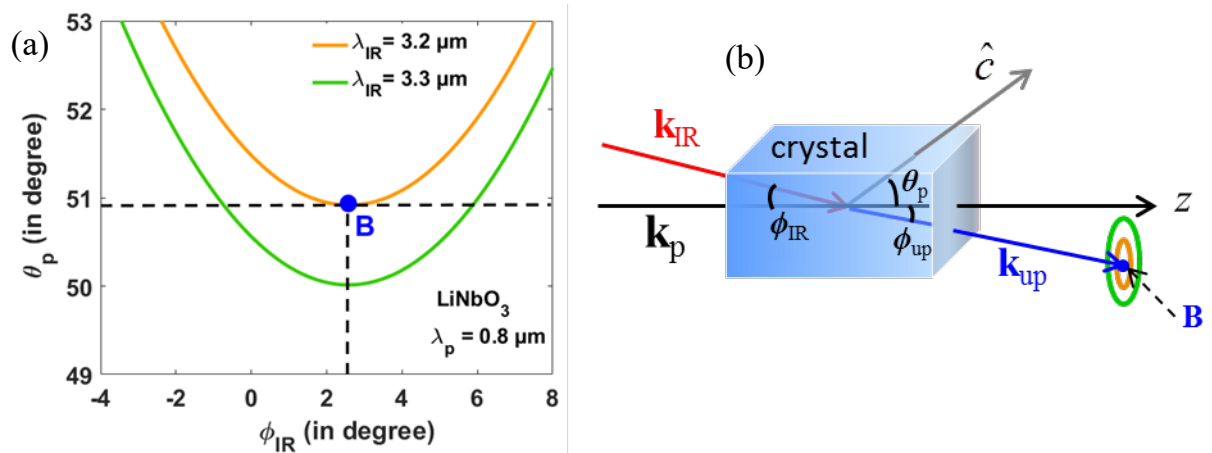

Fig. 13. (a) BPM in a $\mathrm{LiNbO}_{3}$ crystal calculated (by Ashik A. S.) for a fixed $\lambda_{\mathrm{p}}=0.8 \mu \mathrm{m}$ and two $\lambda_{\mathrm{IR}}=3.2 \mu \mathrm{m}$ (orange) and $3.3 \mu \mathrm{m}$ (green). Point B indicates the non-collinear tangential PM point. (b) Schematic representation of $\mathbf{k}$-vectors for non-collinear interaction in a bulk nonlinear crystal. Different IR wavelengths satisfy PM along spatial ring patterns of varying radii concentric around point B (indicated by dashed arrow). Different colors for the ring patterns indicate different IR wavelengths that are upconverted.

\section{Noise properties of upconversion}

As for classical imaging systems, a standard figure of merit such as signal-to-noise ratio (SNR) and noise equivalent power (NEP) can also be used for upconversion systems. In this section, a brief discussion on the noise sources and the SNR for a given upconversion efficiency is discussed. For direct detection, the optical shot noise (OSN) coming from the IR signal (object) is typically the limiting case for bright signals. The OSN is proportional to $\sqrt{N_{\text {photon }}}$, where $N_{\text {photon }}$ is the photon count of the IR signal. However, for detection of faint IR signals (low signal level, for e.g. LIDAR or microscopy applications), the OSN may be neglected in comparison to other noise sources (typically the background noise originating from the blackbody radiation and thermal of the detector itself). For upconversion detection, the thermal noise of the detector material (e.g. silicon) generally does not contribute significantly to the dark noise; however, during the parametric process, noise is inevitably added to the signal. Thus, any noise originating from the final detector (dark noise) and the optical noise originating from the upconversion process itself (up-noise) have to be combined in order to evaluate the overall system performance. The SNR can be estimated as [105]

$$
S N R=\frac{i_{\text {signal }}}{\sqrt{\sigma_{\text {up-noise }}^{2}+\sigma_{\text {det }}^{2}}}
$$

where $i_{\text {signal }}$ is the photocurrent driven by the upconverted IR signal. The $\sigma_{\text {up-noise }}$ includes the shot noise originating from the upconverted IR signal and the up-noise (see the sub-section below). The $\sigma_{\text {det }}$ is the dark noise from the final detector. These terms are expressed as

$$
\begin{gathered}
i_{\text {signal }}=\frac{P_{\mathrm{IR}}}{\hbar \omega_{\mathrm{IR}}} \eta_{\text {system }} q ; \sigma_{\text {up-noise }}= \\
\text { where } i_{\text {up-noise }}=\frac{P_{\text {up-noise }} \eta_{\text {det }} \eta_{\text {ext-loss }} q .}{\hbar \omega_{\text {up }}} .
\end{gathered}
$$

$P_{\text {IR }}$ is the IR signal power, $P_{\text {up-noise }}$ is the power of up-noise, $\eta$ 's are efficiencies defined in Eq. (11), $q$ is the charge of an electron. The $N E P_{\text {det, }} B$, and $F$ are the NEP, frequency bandwidth, and noise figure of the final detector, respectively (available in commercial detector's data sheet). Typically, higher upconversion efficiency improves the SNR. For detailed calculation steps of SNR and/or NEP see [106], [107]. By calculating or knowing the values of $\eta$, both $i_{\text {signal }}$ 
and $\sigma_{\text {det }}$ can be estimated. In certain applications, the detector noise $\left(\sigma_{\mathrm{det}}\right)$ can be neglected in comparison to the up-noise; particularly in cases where extremely sensitive detectors (e.g. photomultiplier tube (PMT) [105]) are used. To estimate the $i_{\text {up-noise, }}$ optical power contribution of any up-noise source needs to be investigated.

\section{Optical noise generated in an upconversion module (up-noise)}

Apart from the upconverted signal, any light in the detection spectral band coming out of the upconversion module and reaching the final detector contributes to the overall up-noise. The residual pump and/or its second harmonic are typically the strongest contributor. However, these can be easily blocked by spectral filtering as they are outside the spectral band of the upconverted signal [87].

Depending on the spectral range of the IR signal, the nonlinear crystal, and its temperature, the second strongest contribution to the noise comes from the upconverted thermal radiation originating inside the nonlinear crystal due to its imperfect optical transparency. Note that, this is different from the "dark noise" mentioned earlier in this section. In 1974, Estes et al. [108] presented a detailed analysis of the thermal noise contribution for an IR signal at $10.6 \mu \mathrm{m}$ upconverted in a proustite crystal at room temperature. The relatively high optical absorption of proustite at $10.6 \mu \mathrm{m}$ leads to a $\mu \mathrm{W}$ level of up-noise power in their experimental setup. Later on, the spatial and spectral distribution of such thermal noise have been theoretically estimated and experimentally demonstrated using $\mathrm{LiNbO}_{3}$ and $\mathrm{LiIO}_{3}$ crystals for narrowband as well as broadband upconversion [109]-[112]. Recently, Barh et al. [109] measured a total upconverted thermal noise power of $\sim 30 \mathrm{pW}$ at room temperature for broadband upconversion in the $3.5-$ $5 \mu \mathrm{m}$ range in a bulk PPLN crystal using a $1064 \mathrm{~nm} \mathrm{CW}$ pump. As the thermal radiation is broadband (see Fig. 14) and can be phase matched over a wide range of angles, its contribution can be minimized by using both spatial and spectral filtering. Furthermore, the thermal noise can be reduced by considering a lower average pump power [109]. In that direction, a synchronously pulsed pump and pulsed infrared signal would likely improve the upconversion SNR.

In the highly transparent region of the nonlinear crystal, the aforementioned thermal contribution can be neglected. In that case, the parasitic optical processes inside the nonlinear crystal become the dominant optical noise sources. One such phenomenon is spontaneous parametric downconversion (SPDC) of the pump. SPDC is an incidental second order parametric process in an upconversion crystal, where a pump photon spontaneously splits/downconverts into two lower energy photons $\left(\lambda \operatorname{SPDC}>\lambda_{\mathrm{p}}\right)$ as a result of vacuum fluctuations known from quantum mechanics [113]. However, the SPDC process is non-phase matched since the upconverter is designed to fulfill the PM condition for the upconversion process. Therefore, the intensity of the SPDC is usually very low, but in a poled crystal (e.g., PPLN) the SPDC intensity can be relatively high depending on its poling quality given by its random duty cycle (RDC) error [114]. When a short-wavelength pump is used, i.e. $\lambda_{\mathrm{IR}}>\lambda_{\mathrm{p}}$, the broadband SPDC induced by the pump spectrally overlaps with the IR signal. This part of the SPDC spectrum then undergoes wavelength translation through upconversion process just like the desired signal and hence contributes as optical noise (see Fig. 14). In 1969, Tang [115] described this phenomenon by treating it as a quantum mechanical scattering problem and estimated the noise power of upconverted SPDC (USPDC). Later on researchers have measured USPDC photon count rates of about $10^{5} / \mathrm{sec}$ in PPLN based upconverter for $1550 \mathrm{~nm}$ [83], [105]. Recently, Meng et al. [116] measured the spatial distribution of the USPDC noise. They proposed a spatial filtering scheme using a pinhole and temperature tuning of the phase matching to reduce the USPDC count rate to $\sim 10^{3} / \mathrm{sec}$ for signals in the telecom wavelength range. Such progress is essential towards developing single-photon upconversion detectors. Nevertheless, care must be taken to avoid any undesired circumstances where both up- and downcoversion processes are phase matched [117]. Furthermore, noise investigations in [116] also contribute to the understanding of the up-noise in upconversion imaging systems, which have the same optical noise source as the upconversion detector. In particular, the unique spatial 
distribution of the USPDC noise (see Fig. 15) in the PPLN based upconversion system can lead to a non-uniform noise background for the upconversion imaging.

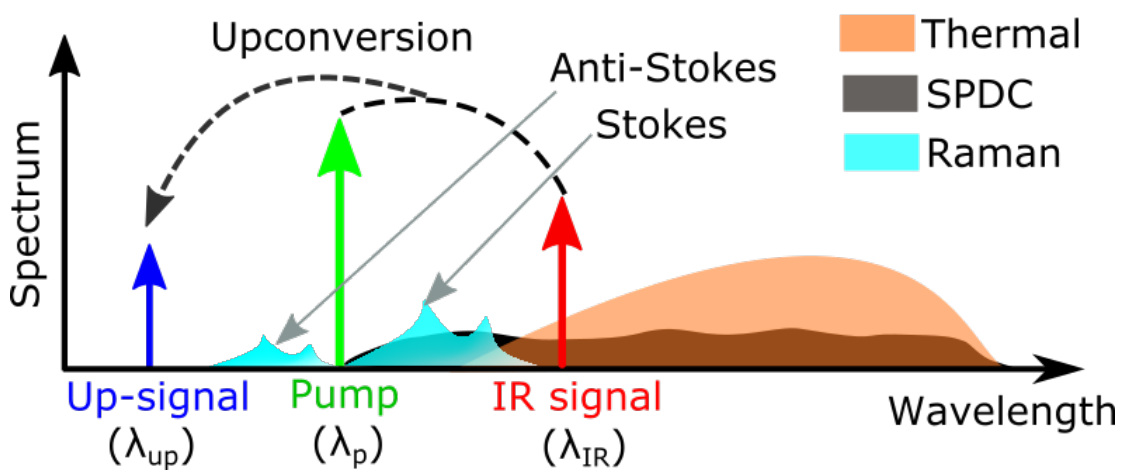

Fig. 14. Schematic spectrum of different optical noise sources in a typical short-wavelengthpumped upconversion module. The spectral position of pump, IR, and upconverted wave are indicated for better understanding. Note that the SPDC and Raman (Stokes and Anti-Stokes) photons are generated due to a strong pump.
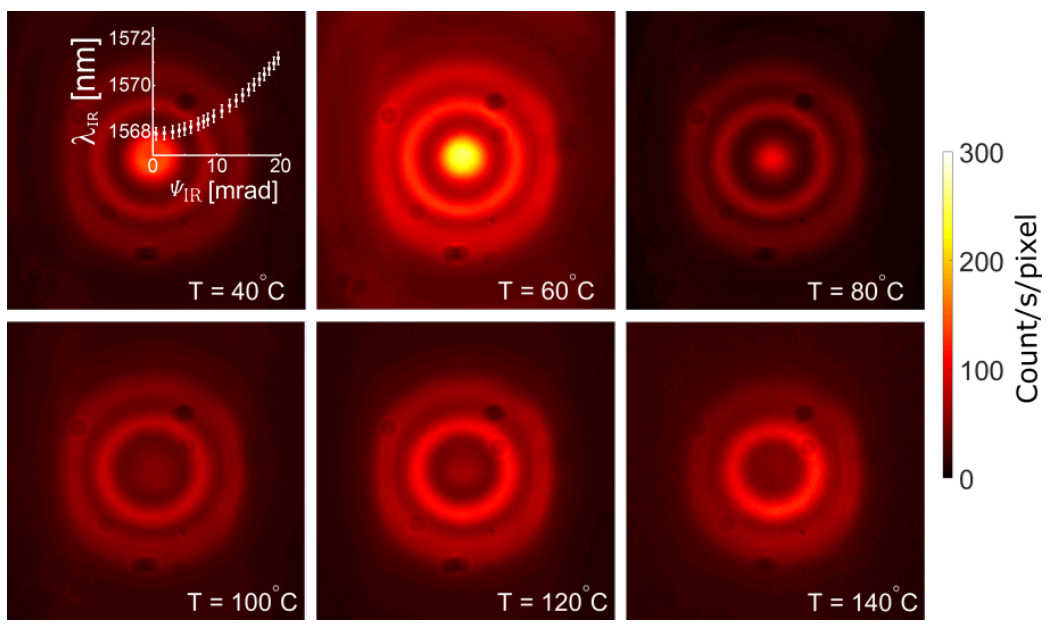

Fig. 15. Spatial distribution of the USPDC noise intensity with a $1064 \mathrm{~nm}$ pumped PPLN crystal operating at different temperatures. The overlay plot for the USPDC noise at $\mathrm{T}=40^{\circ} \mathrm{C}$ shows that the noise varies radially not only in intensity but also in wavelength, $\lambda_{\mathrm{IR}}=\lambda_{\mathrm{p}} \lambda_{\mathrm{USPDC}} /\left(\lambda_{\mathrm{p}}-\right.$ $\lambda_{\text {USPDC }}$. Adapted from [116].

In order to remove the USPDC noise source, Pelc et al. [118] suggested using a "longwavelength-pumping" scheme, where $\lambda_{\mathrm{p}}>\lambda_{\mathrm{IR}}$, to avoid the effect of SPDC (as it appears at the longer wavelength side of the pump spectrum). By doing so, they also got rid of Stokes Raman noise when it is not separated well enough from the pump spectrum (see Fig. 14). In this configuration, mainly the Anti-Stokes Raman contributes to the up-noise. Recently Kuo et al. [119] suggested to cool down the nonlinear crystal to reduce the Raman contribution significantly. They predicted a noise count level of $250 / \mathrm{sec}$ for a PPLN crystal cooled down to $-40^{\circ} \mathrm{C}$. At this low noise level, upconversion can be used as a quantum conversion device. In addition to low up-noise, higher upconversion efficiency $\left(\eta_{\mathrm{up} \rightarrow \mathrm{IR}}\right)$ is desirable to achieve even higher SNR.

For imaging where non-collinear phase matching is typically considered in order to increase the image FoV, the upconversion efficiency of a monochromatic input IR signal depends on the limited angular acceptance bandwidth (sub-sections 3.3) and reduced effective crystal length [112]. The reduced $\eta_{\text {up }}$ consequently reduces the SNR. The Gaussian intensity 
distribution of the pump imposes lower pump intensity for the off-axis IR photons in the nonlinear crystal [69]. Thus, there is a trade-off between imaging FoV, efficiency, and SNR. In 1970's upconversion based imaging experiment, the photomultiplier's dark noise and background noise from the photographic plate (imaging) were the limiting noise sources [27], [31] for low average pump power. In 1977, Boyd and Townes [27] estimated the NEP of each spatially resolvable element on their photographic plate to be $\sim 3 \times 10^{-10} \mathrm{~W} / \mathrm{Hz}^{1 / 2}$. In 1978 Krishnan et al. made a comparative test on SNR for direct and upconversion based IR imaging systems [50]. They concluded that direct detection was superior to the upconversion detection both for thermal imaging and active illumination-based imaging. In the best case (active illumination), direct detection ( $\mathrm{HgCdTe})$ gave an $\mathrm{SNR} / \mathrm{sec}$ of $200 \mathrm{sec}^{-1}$ where upconversion gave an SNR/sec of $\sim 20 \mathrm{sec}^{-1}$ for detection in the $8-12 \mu \mathrm{m}$ range. Note that, in [50] SNR values are given in $\mathrm{sec}^{-1}$ as pulsed illumination was considered for range-gating application. In a modern experimental scenario, the sensitivity of the upconversion detection has been improved significantly by using highly sensitive CCD or PMT as the final detector for upconversion imaging. Imaki and Kobayashi [44] have measured the minimum detectable IR power (at $3.4 \mu \mathrm{m}$ ) for an $\mathrm{SNR}=1$ to be $\sim 2.0 \times 10^{-10} \mathrm{~W}$ with a PPLN based upconversion imaging device. They compared this sensitivity with a traditional InAs detector, cooled at $-40^{\circ} \mathrm{C}$ and concluded that the upconversion device yields 11 times higher sensitivity than the cooled InAs. Dam et al. [45] demonstrated single-photon imaging at an IR range of $3 \mu \mathrm{m}$, where they estimated an up-noise as low as 0.2 photons/spatial element/sec in a PPLN based intracavity upconverter. Very recently, Pedersen et al. [107] compared the NEP of an upconversion detector and a cooled $\mathrm{HgCdTe}$ detector in the $3 \mu \mathrm{m}$ range. With their upconversion detector having an overall detection efficiency (upconversion module + silicon detector, $\eta_{\text {system }}$ ) of $2 \%$, they obtained a NEP of $20 \mathrm{fW} / \mathrm{Hz}^{1 / 2}$ at $3.39 \mu \mathrm{m}$, which is 50 times better than the state-of-theart cooled $\mathrm{HgCdTe}$ detector of much higher efficiency $66 \%$. Furthermore, with an upconversion efficiency $\eta_{\text {up }}$ of only $6 \%$, they estimated the internal NEP of the upconverter itself to be $2.3 \mathrm{fW} / \mathrm{Hz}^{1 / 2}$ at $3.39 \mu \mathrm{m}$. Thus, upconversion detector is widely suitable for high-sensitive low IR signal detection. Furthermore, upconversion detection can exploit the wide temporal bandwidth of silicon detectors (multiple $\mathrm{GHz}$ ), whereas the best available $\mathrm{HgCdTe}$ detectors only reach $10-100 \mathrm{MHz}$ scale (e.g. Vigo system). For further details, a list of measured/estimated noise properties of upconversion based imaging is given at the end of section 7 .

\section{Design considerations for upconversion imaging}

In this section we thoroughly discuss different parameters which are crucial for designing an upconversion imaging setup including some historical perspectives.

\subsection{Choice of nonlinear crystal}

A crucial optical element is the nonlinear crystal, which has to be properly selected for a given application, as many different properties of the crystal play a role in the nonlinear conversion process. In general, a crystal with high second order nonlinearity is preferred. The linear refractive index, $n$ at the optical wavelengths of commonly used nonlinear materials lies in the range of $2-4$. Thus, such small variation in $n$ does not directly affect the upconversion efficiency in a significant manner according to Eq. (10). However, Miller's rule says "...the crystals that will exhibit large nonlinear effects are those with large linear susceptibilities..." [120]. Thus, a larger value of $n$ generally yields a larger nonlinear coefficient. The effective nonlinear coefficient, $d_{\text {eff }}$ depends on the direction of polarization of the interacting waves in the crystal, which differs for different types of interaction. The details for calculating $d_{\text {eff }}$ can be found in textbooks [61], [99]. In the following, some other important material properties, that are vital for designing an upconversion imaging system are considered. 
Only non-centrosymmetric crystals [60] possess non-zero second order nonlinear coefficient. Thus, for upconversion non-centrosymmetric crystals must be chosen.

A large crystal aperture $\left(\sim 5 \times 5 \mathrm{~mm}^{2}\right.$ or larger $)$ is typically used as it allows for high spatial resolution or FoV of the upconversion imaging system (as discussed in section 2). Thus, bulk crystals with larger aperture size are best suited for upconversion imaging.

An optically transparent crystal at all three wavelengths $\left(\lambda_{\mathrm{p}}, \lambda_{\mathrm{IR}}\right.$, and $\left.\lambda_{\text {up }}\right)$ is necessary to achieve very low linear loss and thermal noise in the upconversion process. Especially for intracavity setups, where the pump wave is resonant, the linear loss at $\lambda_{\mathrm{p}}$ must be in the order of $1 \%$ or less to obtain a high circulating power in the cavity. The first upconversion imaging was performed in a lithium niobate $\left(\mathrm{LiNbO}_{3}\right)$ bulk crystal using a single-pass pulsed pump configuration [19]. After a decade, a $\mathrm{LiNbO}_{3}$ bulk crystal was incorporated into an intracavitybased system owing to its low loss at $\lambda_{\mathrm{p}}$ [40]. Nowadays, the optical quality of $\mathrm{LiNbO}_{3}$, as well as the anti-reflection coating at the pump wavelength, are significantly improved such that an intracavity CW power of hundreds of Watts can easily be obtained [45]. Oxide-based crystals, like $\mathrm{LiNbO}_{3}$, are not suitable for imaging at MIR wavelengths above $\sim 5 \mu \mathrm{m}$ due to its high absorption at longer wavelengths. Moving further into the IR, chalcogenide (S, Se, Te) based crystals, e.g. proustite crystal $\left(\mathrm{Ag}_{3} \mathrm{AsS}_{3}\right)$, were the main choice in the 1970's imaging experiments [20], [25], [27], [121]. Later on several chalcogenide- based crystals were proposed [122] and used for upconversion [100], [123]-[125].

Low two-photon absorption (TPA) at the pump wavelength is necessary to reduce losses. As mentioned above, chalcogenide-based crystals are transparent at MIR; however, they suffer from high TPA due to their small bandgap. In particular, for materials with bandgap below 2 $\mathrm{eV}$ (e.g. $\mathrm{AgGaSe}_{2}, \mathrm{ZnGeP}_{2}$ ), pumping at the near-IR wavelength (e.g. at $1 \mu \mathrm{m}$ ) is impossible due to strong TPA [122]. Researchers have shown that Li- [126] and Ba- [127] ion based chalcogenide possess higher bandgap. However, their use in upconversion is still in its infancy with only a few reported results [124], [128].

High laser damage threshold (LDT) at the pump wavelength is desirable for high peak power pulsed or intracavity based operation. Thus, care should be taken while designing the pump parameters (focus spot size, power level, etc.). Also, the LDT for the surface is generally lower than the bulk material. Hence, care should be taken while focusing a laser beam into the crystal. Nowadays, most common nonlinear crystals have LDT of around $100 \mathrm{MW} / \mathrm{cm}^{2}$ for ns pumping. Furthermore, average $(\mathrm{CW})$ pump power limitations are much more severe than peak power pump. The LDT of coatings on crystal facets should also be considered, especially for intracavity setups.

Birefringence is necessary to compensate for the dispersion induced phase mismatch in a BPM configuration (section 3). It is essentially a measure of the difference in refractive index on the direction of polarization at a wavelength in an anisotropic crystal [61]. Many crystals are discarded because of their isotropic nature even if they possess other qualities.

High thermal conductivity, low temperature dispersion $(d n / d T)$ and low thermal expansion make a crystal less prone to the thermal lensing effect [129], which is highly undesirable in imaging applications. In case of materials with low thermal conductivity, the absorbed power cannot dissipate efficiently out of the crystal and hence temperature dependent refractive index gradient is formed, which further increases for materials with high $d n / d T$. The crystal eventually works as a GRIN lens. For example, due to high thermal conductivity, $\mathrm{KTiOPO}_{4}, \mathrm{LiNbO}_{3}, \mathrm{LiInS}(\mathrm{e})_{2}, \mathrm{ZnGeP}_{2}$ can be used in intracavity imaging setups, whereas $\mathrm{AgGaS}_{2}$ or $\mathrm{AgGaSe}_{2}$, both with low thermal conductivity, are more suitable for a single-pass imaging system.

Commonly used nonlinear crystals and some that can be potentially used in upconversion imaging, are listed in Table 3, based on the aforementioned properties.

Table 3. Optical Properties of Nonlinear Crystals Used/Suitable for Upconversion Imaging 


\begin{tabular}{|c|c|c|c|c|c|c|c|c|c|}
\hline 焉 & 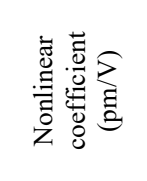 & 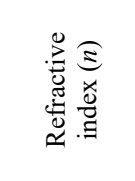 & 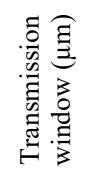 & 它 & 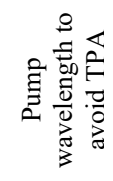 & 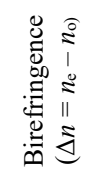 & 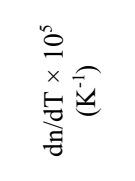 & 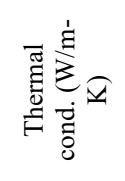 & 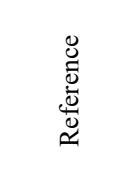 \\
\hline $\begin{array}{c}\text { Proustite } \\
\mathrm{Ag}_{3} \mathrm{AsS}_{3} \\
\text { uniaxial }\end{array}$ & $\begin{array}{l}\text { d22 (18), } \\
\text { d31(11.3) }\end{array}$ & $\begin{array}{l}n_{\mathrm{e}}(2.59) \\
n_{\mathrm{o}}(2.82) \\
@ 1 \mu \mathrm{m}\end{array}$ & $\begin{array}{l}0.6- \\
13\end{array}$ & $\begin{array}{l}4(\mathrm{~ns}) @ \\
1.06 \mu \mathrm{m}\end{array}$ & $\begin{array}{l}\lambda_{\mathrm{p}}> \\
0.6 \\
\mu \mathrm{m}\end{array}$ & -0.2 & ---- & --- & $\begin{array}{l}{[20],} \\
{[22],} \\
{[56],} \\
{[130]}\end{array}$ \\
\hline $\begin{array}{c}\mathrm{KH}_{2} \mathrm{PO}_{4} \\
(\mathrm{KDP}) \\
\text { uniaxial }\end{array}$ & d36 (0.43) & $\begin{array}{l}n_{\mathrm{e}}(1.46) \\
n_{\mathrm{o}}(1.49) \\
@ 1.06 \\
\mu \mathrm{m}\end{array}$ & $\begin{array}{l}0.18 \\
-1.5 \\
\end{array}$ & $10^{4}(\mathrm{~ns})$ & $\begin{array}{l}\lambda_{\mathrm{p}}> \\
0.3 \\
\mu \mathrm{m}\end{array}$ & -0.03 & $\begin{array}{l}-3.94(\mathrm{o}) \\
-2.54(\mathrm{e}) \\
@ 0.6 \mu \mathrm{m}\end{array}$ & 1.9 & $\begin{array}{l}60], \\
{[24],} \\
{[131],} \\
{[99]}\end{array}$ \\
\hline $\begin{array}{l}\mathrm{LiB}_{3} \mathrm{O}_{5} \\
(\mathrm{LBO}) \\
\text { biaxial }\end{array}$ & $\begin{array}{l}\mathrm{d} 31(1.05) \\
\mathrm{d} 32(-1.0) \\
\mathrm{d} 33(0.05)\end{array}$ & $\begin{array}{l}n_{X}(1.57) \\
n_{Y}(1.59) \\
n_{Z}(1.61) \\
@ 1 \mu \mathrm{m}\end{array}$ & $\begin{array}{l}0.16 \\
-2.6 \\
\end{array}$ & $\begin{array}{l}>10^{4}(10 \\
\mathrm{ns}) @ \\
1.064 \mu \mathrm{m}\end{array}$ & $\begin{array}{l}\lambda_{\mathrm{p}}> \\
0.35 \\
\mu \mathrm{m}\end{array}$ & ---- & $\begin{array}{l}-0.93(x) \\
-1.36(y) \\
-0.63(z)\end{array}$ & 3.5 & $\begin{array}{l}{[132]-} \\
{[134]}\end{array}$ \\
\hline $\begin{array}{c}\beta-\mathrm{BaB}_{2} \mathrm{O}_{4} \\
(\mathrm{BBO}) \\
\text { uniaxial } \\
\end{array}$ & $\begin{array}{l}\text { d22 (2.3) } \\
\text { d31(0.16) }\end{array}$ & $\begin{array}{l}n_{\mathrm{e}}(1.54), \\
n_{\mathrm{o}}(1.66) \\
@ 1 \mu \mathrm{m} \\
\end{array}$ & $\begin{array}{l}0.2- \\
3.5\end{array}$ & $\begin{array}{l}1.35 \times 10^{4} \\
(\mathrm{~ns}) @ \\
1.06 \mu \mathrm{m} \\
\end{array}$ & $\begin{array}{l}\lambda_{\mathrm{p}}> \\
0.4 \\
\mu \mathrm{m}\end{array}$ & -0.11 & $\begin{array}{l}-1.66(\mathrm{o}) \\
-0.93(\mathrm{e})\end{array}$ & $\begin{array}{l}1.6(\| c) \\
1.2(\perp c)\end{array}$ & $\begin{array}{l}{[135]-} \\
{[138],} \\
{[99]}\end{array}$ \\
\hline $\begin{array}{l}\mathrm{KNbO}_{3} \\
\text { biaxial }\end{array}$ & $\begin{array}{l}\mathrm{d} 32(20.4) \\
\text { @ } 1 \mu \mathrm{m}\end{array}$ & $\begin{array}{l}n_{X}(2.26) \\
n_{Y}(2.22) \\
n_{Z}(2.12) \\
@ 1 \mu \mathrm{m}\end{array}$ & $\begin{array}{l}0.4- \\
4.5\end{array}$ & $\begin{array}{l}\sim 2 \times 10^{4} \\
(0.7 \mathrm{~ns}) \\
@ 1.054 \\
\mu \mathrm{m}\end{array}$ & $\begin{array}{l}\lambda_{\mathrm{p}}> \\
0.3 \\
\mu \mathrm{m}\end{array}$ & ---- & $\begin{array}{l}2.2(\| a) \\
6.5\left(\|_{c}\right)\end{array}$ & 4 & [72], [99] \\
\hline $\begin{array}{c}\mathrm{KTiOPO}_{4} \\
\text { (KTP) } \\
\text { biaxial }\end{array}$ & $\begin{array}{l}\text { d31 (6.5) } \\
\text { d33 (13.7) }\end{array}$ & $\begin{array}{l}n_{X}(1.74) \\
n_{Y}(1.748) \\
n_{Z}(1.83) \\
@ 1.064 \\
\mu \mathrm{m}\end{array}$ & $\begin{array}{l}0.35 \\
-4.5\end{array}$ & $\begin{array}{l}15 \times 10^{3}(1 \\
\mathrm{ns}) @ \\
1.064 \mu \mathrm{m}\end{array}$ & $\begin{array}{l}\lambda_{\mathrm{p}}> \\
0.45 \\
\mu \mathrm{m}\end{array}$ & ---- & $\begin{array}{l}1.1(x) \\
1.3(y) \\
1.6(z)\end{array}$ & 13 & $\begin{array}{l}{[70],} \\
{[134],} \\
{[139],} \\
{[140]}\end{array}$ \\
\hline $\begin{array}{l}\mathrm{LiNbO}_{3} \\
\text { uniaxial }\end{array}$ & $\begin{array}{l}\text { d32 (-30), } \\
\text { d31 (-5.9) }\end{array}$ & $\begin{array}{l}n_{\mathrm{e}}(2.16) \\
n_{\mathrm{o}}(2.23) \\
@ 1.064 \\
\mu \mathrm{m}\end{array}$ & $\begin{array}{l}0.4- \\
5.5\end{array}$ & $\begin{array}{l}10^{3}(\mathrm{~ns}) \\
@ 1.05 \\
\mu \mathrm{m}\end{array}$ & $\begin{array}{l}\lambda_{\mathrm{p}}> \\
0.5 \\
\mu \mathrm{m}\end{array}$ & -0.08 & $\begin{array}{l}-0.09(\mathrm{o}) \\
+3.91(\mathrm{e}) \\
@ 1.4 \mu \mathrm{m}\end{array}$ & $\sim 5$ & $\begin{array}{l}{[19],} \\
{[141],} \\
{[131]}\end{array}$ \\
\hline $\mathrm{ZnGeP}_{2}$ & $\begin{array}{l}\mathrm{d} 36(79) \\
\text { @ } 4.56 \mu \mathrm{m}\end{array}$ & $\begin{array}{l}n_{\mathrm{e}}(3.3) \\
n_{\mathrm{o}}(3.25) \\
@ 1 \mu \mathrm{m}\end{array}$ & $\begin{array}{l}1- \\
12\end{array}$ & $\begin{array}{l}100(10 \\
\mathrm{ns}) @ \\
2.09 \mu \mathrm{m}\end{array}$ & $\begin{array}{l}\lambda_{\mathrm{p}}> \\
1.5 \\
\mu \mathrm{m}\end{array}$ & 0.05 & $\begin{array}{l}21.18(\mathrm{o}) \\
23.01(\mathrm{e}) \\
(a) 1 \mu \mathrm{m}\end{array}$ & $\begin{array}{l}36(\| c) \\
35(\perp c)\end{array}$ & $\begin{array}{l}{[31],} \\
{[122],} \\
{[131]}\end{array}$ \\
\hline $\begin{array}{l}\mathrm{AgGaS}_{2} \\
\text { uniaxial }\end{array}$ & $\begin{array}{l}\mathrm{d} 36(12.6) \\
@ 10.6 \mu \mathrm{m}\end{array}$ & $\begin{array}{l}n_{\mathrm{e}}(2.4), \\
n_{\mathrm{o}}(2.45) \\
@ 1.06 \\
\mu \mathrm{m}\end{array}$ & $\begin{array}{l}0.47 \\
-13\end{array}$ & $\begin{array}{l}150(5 \\
\mathrm{ns}) @ \\
1.064 \mu \mathrm{m}\end{array}$ & $\begin{array}{l}\lambda_{\mathrm{p}}> \\
0.8 \\
\mu \mathrm{m}\end{array}$ & -0.05 & $\begin{array}{l}16.7(\mathrm{o}) \\
17.6(\mathrm{e}) \\
@ 1.06 \\
\mu \mathrm{m}\end{array}$ & $\begin{array}{l}1.4(\| c) \\
1.5(\perp c)\end{array}$ & $\begin{array}{l}{[122],} \\
{[125],} \\
{[131]}\end{array}$ \\
\hline $\begin{array}{c}\mathrm{AgGaSe}_{2} \\
\text { uniaxial }\end{array}$ & $\begin{array}{l}\mathrm{d} 36(33) \\
@ 10.6 \mu \mathrm{m}\end{array}$ & $\begin{array}{l}n_{\mathrm{e}}(2.68) \\
n_{\mathrm{o}}(2.70) \\
1.06 \mu \mathrm{m}\end{array}$ & $\begin{array}{r}0.76 \\
-18 \\
\end{array}$ & $\begin{array}{l}\sim 25(10 \\
\mathrm{ns}) @ \\
1.06 \mu \mathrm{m}\end{array}$ & $\begin{array}{l}\lambda_{\mathrm{p}}> \\
1.9 \\
\mu \mathrm{m} \\
\end{array}$ & -0.02 & $\begin{array}{l}9.8(\mathrm{o}) \\
6.6(\mathrm{e}) @ \\
1.06 \mu \mathrm{m} \\
\end{array}$ & $\begin{array}{l}1.0(\| c) \\
1.1(\perp c)\end{array}$ & $\begin{array}{l}{[122],} \\
{[142]}\end{array}$ \\
\hline $\begin{array}{c}\mathrm{LiInS}_{2} / \mathrm{Li} \\
\mathrm{InSe}_{2} \\
\text { biaxial }\end{array}$ & $\begin{array}{l}\mathrm{d} 31 \\
(7.3 / 11.8) \\
\mathrm{d} 24 \\
(5.7 / 8.2) \\
(2.3 \mu \mathrm{m} \\
\end{array}$ & $\begin{array}{l}n_{X}(2.171) \\
n_{Y}(2.212) \\
n_{Z}(2.22) \\
@ 0.7 \mu \mathrm{m}\end{array}$ & $\begin{array}{l}0.35 \\
-13\end{array}$ & $\begin{array}{l}\sim 10^{3}(10 \\
\mathrm{ns}) @ \\
1.064 \mu \mathrm{m}\end{array}$ & $\begin{array}{l}\lambda_{\mathrm{p}}> \\
0.8 \\
\mu \mathrm{m}\end{array}$ & ---- & $\begin{array}{l}5.68(x) \\
9.34(y) \\
7.37(z)\end{array}$ & $\begin{array}{l}6.2(x) \\
6.0(y) \\
7.6(z)\end{array}$ & $\begin{array}{l}{[128],} \\
{[143],} \\
{[144]}\end{array}$ \\
\hline $\begin{array}{c}\mathrm{BaGa}_{4} \mathrm{Se}_{7} \\
\text { biaxial }\end{array}$ & $\begin{array}{l}\mathrm{d} 11(18.2) \\
\mathrm{d} 13(20.6) \\
1.064 \mu \mathrm{m}\end{array}$ & $\begin{array}{l}n_{X}(2.486) \\
n_{Y}(2.502) \\
n_{Z}(2.559) \\
@ 1 \mu \mathrm{m}\end{array}$ & $\begin{array}{l}0.78 \\
-15\end{array}$ & $\begin{array}{l}557(5 \\
\mathrm{ns}) @ \\
1.064 \mu \mathrm{m}\end{array}$ & $\begin{array}{l}\lambda_{\mathrm{p}}> \\
0.9 \\
\mu \mathrm{m}\end{array}$ & $\begin{array}{l}0.07 \\
(\max ) \\
@ 1.06 \\
\mu \mathrm{m}\end{array}$ & $\begin{array}{l}5.39(x) \\
5.56(y) \\
5.23(z) \\
1.53 \mu \mathrm{m}\end{array}$ & $\begin{array}{l}0.74(x) \\
0.64(y) \\
0.56(z)\end{array}$ & $\begin{array}{l}{[145]-} \\
{[147]}\end{array}$ \\
\hline $\begin{array}{c}\mathrm{BaGa}_{2} \mathrm{Ge} \\
\mathrm{Se}_{6} \\
\text { uniaxial } \\
\end{array}$ & $\begin{array}{l}\text { d11 } \\
(49 \pm 15)\end{array}$ & $\begin{array}{l}n_{\mathrm{e}}(2.63), \\
n_{\mathrm{o}}(2.52) \\
@ 1 \mu \mathrm{m}\end{array}$ & $\begin{array}{l}0.6- \\
12\end{array}$ & $\begin{array}{l}110(100 \\
\mathrm{ns}) @ \\
10.6 \mu \mathrm{m}\end{array}$ & $\begin{array}{l}\lambda_{\mathrm{p}}> \\
1.08 \\
\mu \mathrm{m}\end{array}$ & $\begin{array}{l}0.08 \sim \\
0.11\end{array}$ & ---- & ---- & $\begin{array}{l}{[127],} \\
{[148]}\end{array}$ \\
\hline OPGAP & d14 (70.6) & $\begin{array}{l}n(3.04) \\
\text { (a) } 2 \mu \mathrm{m}\end{array}$ & $\begin{array}{l}0.7- \\
12.5\end{array}$ & $\begin{array}{l}\sim 56(15 \\
\mathrm{ns}) @ \\
1.064 \mu \mathrm{m}\end{array}$ & $\begin{array}{l}\lambda_{\mathrm{p}}> \\
0.9 \\
\mu \mathrm{m}\end{array}$ & ---- & ---- & $\sim 110$ & $\begin{array}{l}{[79],} \\
{[149]}\end{array}$ \\
\hline OpGaAs & $\mathrm{d} 14(\geq 90)$ & $\begin{array}{l}n(3.3) @ \\
4 \mu \mathrm{m}\end{array}$ & $\begin{array}{l}0.85- \\
18\end{array}$ & $\begin{array}{l}\sim 2 \times 10^{5} \\
(35 \mathrm{ps}, \\
10 \mathrm{~Hz}) @ \\
1.064 \mu \mathrm{m}\end{array}$ & $\begin{array}{l}\lambda_{\mathrm{p}} \geq 2 \\
\mu \mathrm{m}\end{array}$ & --- & $\begin{array}{l}\sim 14.7 @ \\
10 \mu \mathrm{m}\end{array}$ & $\sim 55$ & $\begin{array}{l}{[150]-} \\
{[152]}\end{array}$ \\
\hline
\end{tabular}


Light shade: Crystals have already been used in upconversion imaging at infrared Dark shade: Potential candidate

\subsection{Choice of pump}

In an upconversion process, the upconverted wave always ends up at a shorter wavelength than the two input waves ( $\lambda_{\mathrm{up}}<\lambda_{\mathrm{p}}$ and $\lambda_{\mathrm{IR}}$ ), following the energy conservation condition Eq. (2). Therefore, to detect the upconverted wave using a silicon-CCD, the two input wavelengths $\lambda_{\mathrm{p}}$ and $\lambda_{\mathrm{IR}}$ should be chosen so that the upconverted signal, $\lambda_{\text {up }}$ falls in the silicon detector spectral sensitivity range $(0.3-1 \mu \mathrm{m})$. Pumping at long wavelength, $\lambda_{\mathrm{p}}>\lambda_{\mathrm{IR}}$ is an attractive scheme for low-noise upconversion [70], [118], where the USPDC noise [115] can be avoided. However, this scheme is limited to the NIR signals $(1-2 \mu \mathrm{m})$ detection. In addition, for best quality imaging and most efficient upconversion, the pump should be an intense coherent source (e.g. a laser), which limits the choice of pump wavelengths. In the 1970's imaging experiments, the choice of the pump was limited to either ruby laser at $0.694 \mu \mathrm{m}$ [19], [22], [25] or Nd:YAG laser at $1.06 \mu \mathrm{m}$ [24]. However, the ruby pump was most desirable as it allowed the use of efficient S-20 semitransparent cathode $(300-850 \mathrm{~nm})$ as end detector, whereas Nd:YAG pump required the less sensitive S-1 cathode $(400-1200 \mathrm{~nm})$. In modern days, pumping around 1 $\mu \mathrm{m}$ is the best choice, in order to make use of the highly sensitive Si-CCD $(400-1100 \mathrm{~nm})$, whereas MIR imaging with longer wavelength pumping (e.g. Tm-doped laser at $2 \mu \mathrm{m}$ ) would require the more expensive InGaAs camera $(1000-1700 \mathrm{~nm})$.

Optimal pumping schemes should also be chosen in terms of the temporal profile (CW or pulse) of the input IR signal and the pump as the upconversion efficiency depends on their temporal overlap. If the IR input is thermal radiation or a CW laser (meaning that the signal is always on), the upconversion efficiency is determined by the average pump power $\left(P_{\text {av }}\right)$. In that case a pulsed pump may not be necessary; instead, a high power CW pump would be a better choice. Generally, the output of a cost effective single transverse mode solid state laser (e.g. at $1 \mu \mathrm{m}$ ) is limited to a few Watts, which limits the efficiency in order of $10^{-6}$ [153]. Expensive high power fiber lasers are available, however their output $\mathrm{CW}$ power is still limited to few 10's of W. In 1978, Falk and See [40] suggested and first demonstrated an intracavity upconversion scheme, where the nonlinear crystal $\left(\mathrm{LiNbO}_{3}\right)$ was placed inside the pump laser (Argon laser at $0.514 \mu \mathrm{m}$ ) cavity to gain access to the highest available circulating power. However, their circulating power was limited to several Watts due to high absorption loss (around $12 \%$ in single-pass) of $\mathrm{LiNbO}_{3}$ at $0.514 \mu \mathrm{m}$. The highest measured power conversion efficiency was $3.8 \times 10^{-3}$ at detected infrared wavelength of $3.39 \mu \mathrm{m}$ [40]. A few years later, Schow et al. [154] demonstrated the first intracavity based image upconversion using a proustite crystal as nonlinear medium inside a dye laser cavity $(0.697 \mu \mathrm{m})$ to produce upconverted image of a $\mathrm{CO}_{2}$ laser $(10.6 \mu \mathrm{m})$, however with low resolution and low efficiency (examples are listed at the end of section 7). Note that such intracavity scheme for SHG process was already proposed in 1968 by Geusic et al. [155]. Recent progress on materials, antireflection (AR) coatings and laser designs has resulted in a vast improvement in the intracavity based $\mathrm{CW}$ upconversion systems. An internal conversion efficiency of more than $80 \%$ for point detection at the communication wavelength $(1550 \mathrm{~nm})$ [81], [83] and $20 \%$ for MIR imaging [14] and spectroscopy [87] has been achieved in bulk crystals. For imaging, a single transverse optical mode for the pump laser is an additional requirement to optimize the image resolution [56], [156]. In a $4 f$ imaging setup, the pump laser beam waist in the Fourier plane is typically chosen to match the transverse dimension of the crystal to give the best possible spatial resolution. For a single transverse mode Gaussian beam the confocal parameter must be greater than the crystal length $\left(l_{c}\right)$ to make it plane wave like [63]. Additionally, a single longitudinal mode for the pump ensures better spectral purity. In that case, a ring cavity might be a better choice than a linear cavity [83], [157]. Furthermore, it offers higher damage threshold as the pump laser propagates through the nonlinear material in a unidirectional manner. One drawback of using a CW pump is that the excess pump power can easily heat up the nonlinear crystal. It 
leads to an increase in thermal noise [109] (as discussed in section 4) and thermal lensing effect [158], which can be detrimental in an imaging setup. In that case, nonlinear materials with low $d n / d T$ values (see Table 3 ) should be chosen for intracavity/high pump power related imaging applications.

If the IR signal is pulsed, a synchronously pulsed pump would be a better choice to improve the conversion efficiency and to reduce the noise. The high peak power $\left(P_{\text {peak }}\right)$ of a pulsed pump improves the efficiency while suppresses the need for an intracavity setup. The pump pulse width must be wide enough to cover the entire IR pulse width with a flat response [86]. In 1972 Tseng [26] first demonstrated synchronously pulsed IR upconversion by mixing $1.06 \mu \mathrm{m}$ Nd:YAG laser $\left(P_{\text {peak }}=1 \mathrm{~kW}\right)$ with a $10.6 \mu \mathrm{m} \mathrm{CO}_{2}$ laser $\left(P_{\text {peak }}=740 \mathrm{~W}\right)$ in a $6 \mathrm{~mm}$ long proustite crystal. A peak-to-peak conversion efficiency of $0.84 \%$ was achieved with $8.4 \mathrm{~W}$ of peak power in the upconverted beam. Later several research groups have exploited this technique using new materials [100], [123] and new IR sources [104], [159] for coincidence single-photon detection (efficiency $>90 \%$ ) [160], for low-noise IR spectroscopy [82], [161] and for imaging [97], [104], [162]. For a synchronously pulsed upconversion, the average output power of upconverted light is given by (for flat top temporal pulse profile)

$$
P_{\text {ave }}^{\text {up }} \propto \frac{P_{\text {ave }}^{\mathrm{IR}} P_{\text {ave }}^{\mathrm{p}}}{f_{\text {rep }} \tau_{\text {pulse }}},
$$

where $f_{\text {rep }}$ and $\tau_{\text {pulse }}$ are the repetition rate and pulse temporal width, respectively. Thus, for a given average power (both for IR and pump) low duty cycle conversion improves the efficiency. Furthermore, going from top-hat temporal pulses to Gaussian temporal pulses, the efficiency is reduced by a factor of $\sqrt{2}$. On the other hand, the optical IR noise (e.g. thermal) is $\mathrm{CW}$, and the upconverted thermal noise remains constant assuming the upconversion efficiency is proportional to the pump power. Thus, lowering the duty cycle can improve the SNR for upconversion detection as long as the arrival time of the IR pulses are well known [163]. Generally, synchronous pumping is achieved by incorporating a mechanical delay [164], however, recent publication shows that it can be done electronically [165], discarding the need for any complex mechanical/optical alignment.

\subsection{Coherent and incoherent IR illumination}

A light field is associated with two types of coherence properties. The temporal coherence is defined by the linewidth $(\Delta \omega)$ of a light source. In other words, it characterizes how well a wave can interfere with itself at different time delays. The larger the range of frequencies a source contains, the faster the phase decorrelates, resulting in shorter temporal coherence time $\left(\tau_{\mathrm{c}}\right)$. Thus, there exists a tradeoff: $\tau_{\mathrm{c}} \Delta \omega \leq 1 / 2 \pi$ [68]. The spatial coherence is the crosscorrelation between two spatial points in a light wave at all times. If a wave has a constant complex field amplitude over an infinite extent (i.e. a plane wave), it is perfectly spatially coherent. A point source, say from a pinhole or from a single-mode fiber is also treated as a spatially coherent source. In case of spatially coherent light, the spatial distribution is given by a complex-valued field amplitude, whereas for incoherent light, the spatial distribution is given by a real-valued intensity [68]. A few examples of coherent and incoherent light sources are given in Table 4.

Table 4. Examples of Coherent and Incoherent Light Sources

\begin{tabular}{ccc}
\hline Temporal & Coherent & Incoherent \\
\hline Coherent & Laser (DPSS) & Random laser, laser illuminated diffuser
\end{tabular}


In upconversion imaging, the IR illumination can be either spatially coherent or spatially incoherent (see Table 4 for examples). For the case of spatially coherent IR illumination, the convolution in Eq. (35) has to be performed between the complex electric field of the object and the Fourier transform of the pump. Consequently, the intensity distribution of the image (Iup-image) can be calculated by taking the square of the convolution. Considering a Gaussian pump, Iup-image can be expressed as [70]

$$
\begin{aligned}
I_{\text {up-image }}\left(x^{\prime}, y^{\prime}, \Delta k_{\mathrm{z}}\right) & =\frac{8 \pi d_{\mathrm{eff}}^{2} f_{1}^{2} l_{c}^{2} \lambda_{\mathrm{IR}}^{2}}{n_{\mathrm{p}} n_{\text {up }} f_{2}^{2} \lambda_{\text {up }}^{4} w_{0}^{2}} P_{\mathrm{p}} \operatorname{sinc}^{2}\left(\Delta k_{\mathrm{z}}\left(-\frac{\lambda_{\mathrm{IR}} f_{1}}{\lambda_{\text {up }} f_{2}} x^{\prime},-\frac{\lambda_{\mathrm{IR}} f_{1}}{\lambda_{\text {up }} f_{2}} y^{\prime}\right) \frac{l_{c}}{2}\right) \\
& \times \mid A_{\text {object }}\left(-\frac{\lambda_{\mathrm{IR}} f_{1}}{\lambda_{\text {up }} f_{2}} x^{\prime},-\frac{\lambda_{\mathrm{IR}} f_{1}}{\lambda_{\text {up }} f_{2}} y^{\prime}\right) \otimes\left(\left.\frac{\pi w_{0}^{2}}{\lambda_{\text {up }}^{2} f_{2}^{2}} \exp \left(-\frac{\pi^{2} w_{0}^{2}\left(x^{\prime 2}+y^{\prime 2}\right)}{\lambda_{\text {up }}^{2} f_{2}^{2}}\right)\right|^{2(35)}\right.
\end{aligned}
$$

For a spatially incoherent IR illumination, the convolution has to be performed between the real-valued intensity of the object ( $I_{\mathrm{object}}$ ) and the intensity distribution of the Fourier transform of the pump. Consequently the intensity of the image can be expressed as [166]

$$
\begin{aligned}
& I_{\text {up-image }}\left(x^{\prime}, y^{\prime}, \Delta k_{\mathrm{z}}\right)=\frac{8 \pi^{2} d_{\mathrm{eff}}^{2} l_{c}^{2} \lambda_{\mathrm{IR}}^{2}}{n_{\mathrm{IR}} n_{\mathrm{p}} n_{\text {up }} c \varepsilon_{0} f_{2}^{2} \lambda_{\text {up }}^{4}} P_{\mathrm{p}} \operatorname{sinc}^{2}\left(\Delta k_{\mathrm{z}}\left(-\frac{\lambda_{\mathrm{IR}} f_{1}}{\lambda_{\text {up }} f_{2}} x^{\prime},-\frac{\lambda_{\mathrm{IR}} f_{1}}{\lambda_{\text {up }} f_{2}} y^{\prime}\right) \frac{l_{c}}{2}\right) \\
& \times\left(I_{\text {object }}\left(-\frac{\lambda_{\mathrm{IR}} f_{1}}{\lambda_{\text {up }} f_{2}} x^{\prime},-\frac{\lambda_{\mathrm{IR}} f_{1}}{\lambda_{\text {up }} f_{2}} y^{\prime}\right) \otimes\left(\frac{2 \pi w_{0}^{2}}{\lambda_{\text {up }}^{2} f_{2}^{2}} \exp \left(-\frac{2 \pi^{2} w_{0}^{2}\left(x^{\prime 2}+y^{\prime 2}\right)}{\lambda_{\text {up }}^{2} f_{2}^{2}}\right)\right)\right)
\end{aligned}
$$

For a polychromatic IR illumination, each wavelength can be treated individually to get a set of monochromatic (intensity) images and their linear superposition gives the polychromatic upconverted image.

\subsection{Achieving wide FoV imaging}

In this section, upconverted image formation on a 2-D array-based detector with a certain FoV (angular) is considered and remedies for achieving wide FoV imaging is discussed.

Unlike in direct imaging, the PM requirements (section 3) impose a constraint on the monochromatic $\mathrm{FoV}$ of an upconversion imaging system, as both are related to the input angles of the imaging system. Nevertheless, wider FoV is often desired. In the case of monochromatic IR illumination, the associated $\lambda_{\mathrm{IR}}$ gets phase matched at a certain angle, $\psi_{\mathrm{IR}}$ (PM angle between pump and IR, external to the crystal, shown in Fig. 16). Therefore, even if the entire object plane is illuminated by that IR wave, only a fractional area of it corresponding to the PM acceptance angle (see sub-section 3.3) $\Delta \psi_{\mathrm{IR}}$ (indicated by the red dotted line in Fig. 16) is upconverted and forms a ring patterned intensity in the image plane (indicated by blue dotted lines). As a consequence, $\Delta \psi_{\text {IR }}$ defines the FoV of the imaging system for a fixed PM condition. 


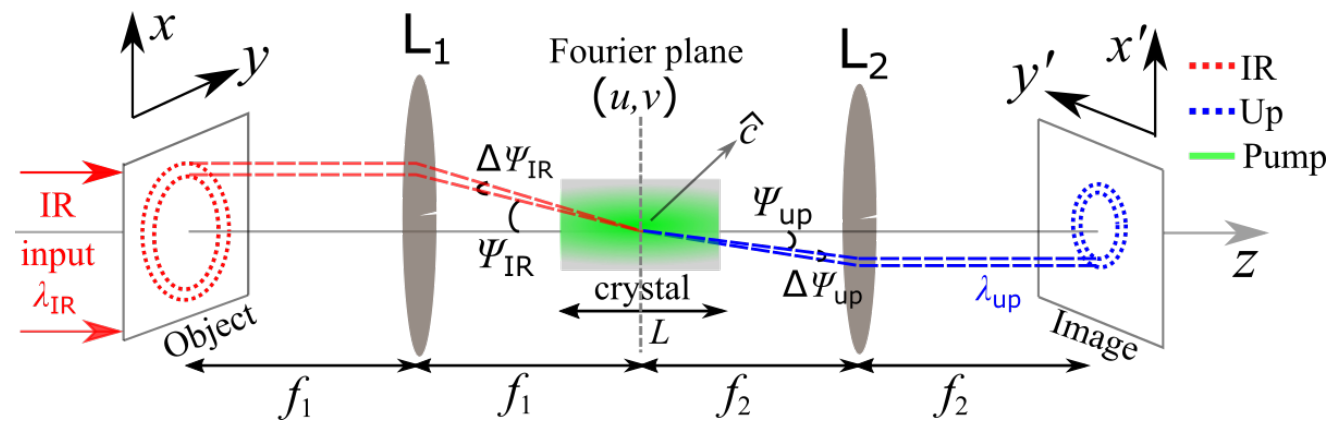

Fig. 16. FoV (region bounded by dotted concentric circles) of an upconversion imaging system for monochromatic IR illumination.

Due to anisotropy in the nonlinear crystal, the propagating light (specifically the $e$-ray) experiences slightly different refractive indices in the $x$ - and $y$-directions. This introduces (i) a slight magnification error from Snell's law going in and out of the crystal if either IR and/or upconverted wave is extraordinary polarized, and (ii) a slight ellipticity to the ring pattern for the upconverted wave. Nevertheless, an object such as that in Fig. 17(a) would have a corresponding upconverted image that is a spatially masked-version of the object as shown in Fig. 17(b). The intensity profile of the apparent mask is mathematically described by Eq. (9). Hence, the $\mathrm{FoV}$ of the upconversion imaging system is limited to annular regions determined by the apparent mask.

(a)

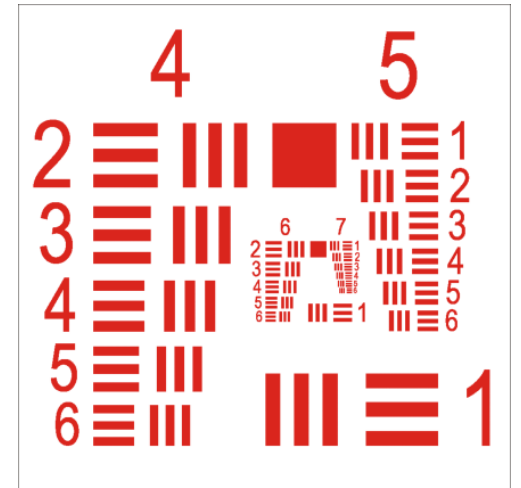

(b)

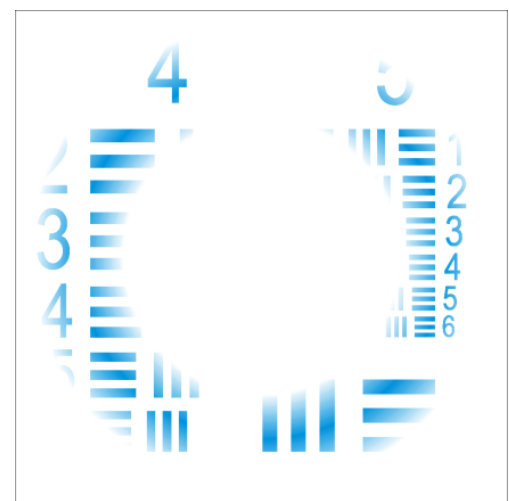

Fig. 17. Image of (a) an object at $\lambda_{\mathrm{IR}}$, and (b) the corresponding upconverted image at $\lambda_{\text {up }}$ with an annular FoV given by the PM condition ( $M=1$ is assumed, i.e. no image inversion).

From the knowledge of different PM conditions, the tangential PM and the NCPM yield a wider FoV (wider $\Delta \psi_{\mathrm{IR}}$ ) in comparison to the $\mathrm{CPM}$ configuration. For example, in a ruby laser pumped $1 \mathrm{~cm}$ long KDP crystal, the achieved FoV was $2.5^{\circ}$ and $3.6^{\circ}$ under the CPM and NCPM condition, respectively [23]. Furthermore, the thickness of the ring, i.e. the $\Delta \psi_{\mathrm{IR}}$, varies with its radius, hence the FoV changes with radial position [164].

\section{Full FoV upconverted image with monochromatic IR illumination}

To get a full FoV monochromatic imaging of an object, one approach is to tune the PM condition for the input IR signal (at $\lambda_{\mathrm{IR}}$ ) in combination with post-processing. The PM condition can be tuned by changing the refractive indices, which can be done by varying the operating temperature [45], [47], [69], [72], [167] or by rotating the crystal [22]-[24], [27], [67], [104], [125]. For example, Junaid et al. [125] demonstrated angle tuning of a bulk $\mathrm{AgGaS}_{2}$ crystal (cut-angle $48^{\circ}$ ), showing an increased FoV by rotating the crystal rotation angle with respect to the $z$-axis, while illuminating the object with a monochromatic beam from a quantum cascade laser operating at $6 \mu \mathrm{m}$ (see Fig. 18). In this example [125], for a pump power of $1.5 \mathrm{~W}$ and 
input IR power of $4.5 \mathrm{~mW}$, the camera exposure time was set at $10 \mathrm{~ms}$ for each crystal rotation angle. Similarly, by changing the temperature of a PPLN crystal, a different annular part (FoV) of the object (illuminated by a narrowband laser operating at $3 \mu \mathrm{m}$ ) can be imaged, as shown in Fig. 19 [45], where camera exposure time was set at $10 \mathrm{sec}$ for each crystal temperature setting. Both techniques need post-processing to combine different sections of the image to produce the final monochromatic image with full FoV.
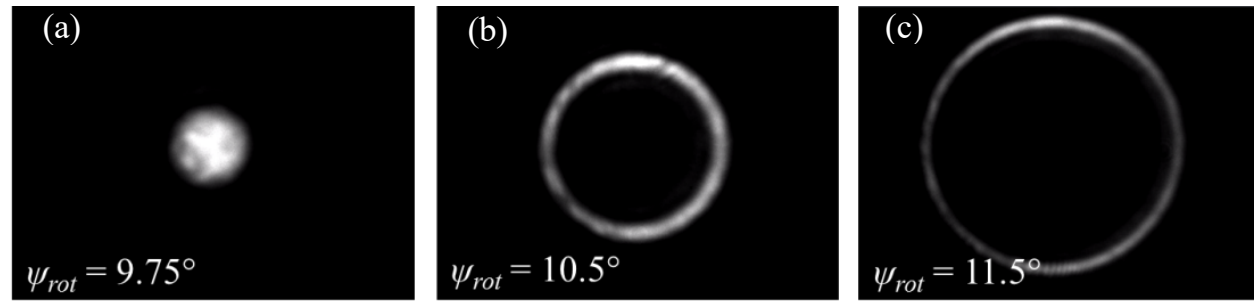

Fig. 18. Adapted from [125] (a)-(c) The acquired upconverted images of the object illumination from a $6 \mu \mathrm{m}$ QCL for different rotation angles (external) $\psi_{\text {rot }}=9.75^{\circ}, 10.5^{\circ}$, and $11.5^{\circ}$. The images are normalized in size.
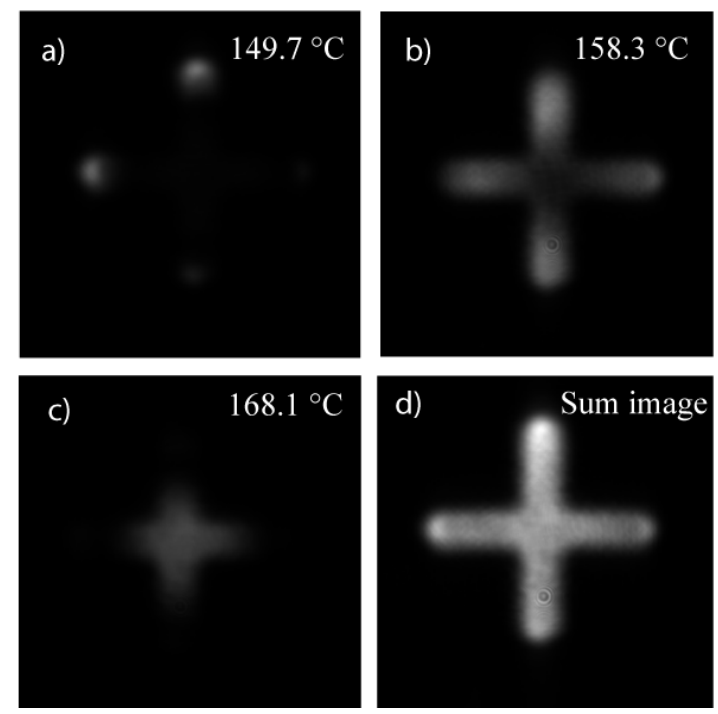

Fig. 19. Adapted from [45]. Monochromatic image reconstruction (a-d) in an intracavity PPLNupconverter. This is demonstrated by adding images (a)-(c) for different crystal temperatures (indicated in each figure) to generate a monochromatic image (d). The cross target was illuminated by a source of wavelength $\lambda_{\mathrm{IR}}=3 \mu \mathrm{m}$.

Recently, Demur et al. [95] proposed an alternative scheme for increasing the FoV by tailoring the pump spectrum. Instead of a monochromatic laser, they used a broadband (few $\mathrm{nm}$ wide) laser to upconvert the entire object (wide FoV), illuminated by a CW laser at $1563 \mathrm{~nm}$, in a single acquisition (acquisition time $=20 \mu \mathrm{s}$ ). They used a PPLN crystal to satisfy the QPM condition for the upconversion process. In this configuration, different pump wavelengths of the broadband pump satisfy the QPM for the same $\lambda_{\mathrm{IR}}$, but for different angles of IR signal (see Fig. 20(a)). According to their calculation, "the angular field-of-view increases as the square root of the pump spectral broadening $\delta \lambda_{\mathrm{p}}$ ". They demonstrated an increase in FoV from 32 mrad to 111 mrad by changing the pump spectrum from monochromatic to polychromatic ( 2.7 $\mathrm{nm}$ wide spectrum); see Fig. 20(b)-(d). This technique does not require any post-processing to get the wide FoV image. However, it should be noted that, though the input IR illumination is monochromatic, the upconverted image is (slightly) polychromatic, satisfying the energy conservation condition (see Eq. (2)), and hence a broader pump spectrum would introduce 
different magnification for different regions in the image. Furthermore, in calculating the upconversion efficiency, one should consider an effective pump power equal to the product of the pump power spectral density and the position-dependent acceptance bandwidth of the upconversion process rather than just the total pump power, as well as a temporally stable system in terms of spectral distribution of the pump.
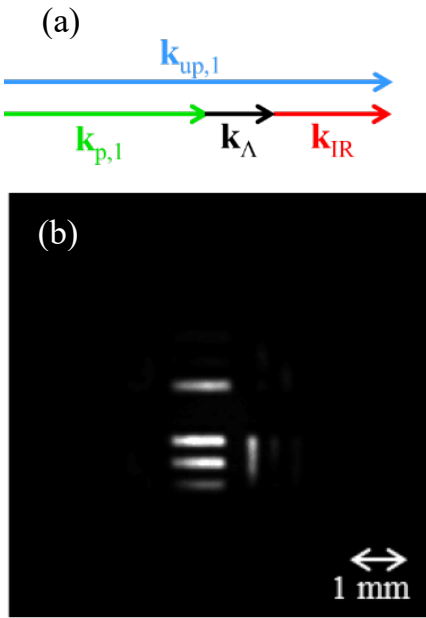
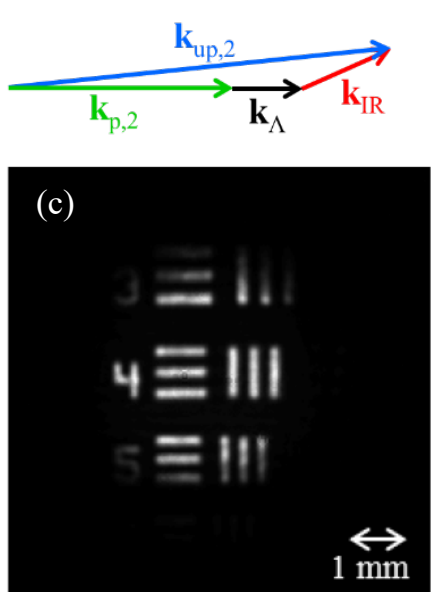
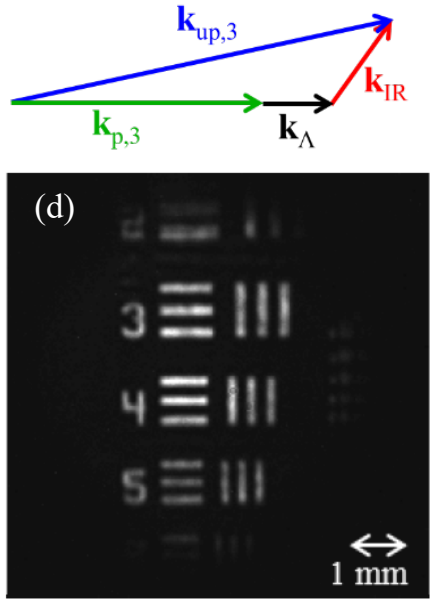

Fig. 20. (a) Schematic diagram showing the quasi phase matching conditions for three pump wavevectors $\left(\left|\mathbf{k}_{\mathrm{p}, \mathrm{i}}\right| \propto 1 / \lambda_{\mathrm{p}, \mathrm{i}}\right)$. The $\mathbf{k}_{\Lambda}$ is the PPLN grating wavevector. According to energy conservation, different upconverted wavelengths are generated corresponding to different pump wavelengths. (b-d) Adapted with permission from [95] (C) The Optical Society. Upconverted images with (b) monochromatic pumping (1 kW peak power), (c) pump of $1 \mathrm{~nm}$ spectral bandwidth $(320 \mathrm{~W}$ peak power), and (d) pump of $2.7 \mathrm{~nm}$ spectral bandwidth $(320 \mathrm{~W}$ peak power).

In 1969, Andrews [23] suggested a continuous scanning technique to acquire a monochromatic image from a monochromatic object with wide FoV and without postprocessing, similar to Fig. 18, where different rotation angles of the crystal give different angular PM conditions. Andrews suggested acquiring all the images (for different rotation angles) within a single acquisition time. He estimated that almost $20^{\circ} \mathrm{FoV}$ is achievable in a $\mathrm{KDP}$ crystal just by scanning it $\sim 4^{\circ}$. Although the technique is quite promising, one needs to take care of image distortion associated with the refraction in the crystal surfaces as it is scanned. Very recently Junaid et al. [168] used such scheme to demonstrate a full FoV (10 mm object diameter) monochromatic imaging at around $3 \mu \mathrm{m}$ (OPO source) acquired within $2.5 \mathrm{~ms}$ using a bulk $\mathrm{LiNbO}_{3}$ crystal. In 2016, Maestre et al. [169], [170] proposed an alternative approach for increasing the monochromatic FoV. They used a temperature gradient along the length of nonlinear crystal (PPLN) to increase the FoV significantly by widening the QPM bandwidth. However, the system needs to be very accurately temperature stabilized. Other ways include spatial translation of the object plane [94], use of microlens arrays (MLAs) instead of usual macroscopic lenses [92].

\section{Full FoV upconverted image with polychromatic IR illumination}

For broadband IR illumination, the upconversion parameters can be designed such that different wavelengths, $\lambda_{I R}$ get phase matched at different IR input angles $\left(\psi_{\mathrm{IR}}\right)$ for a fixed pump (i.e. single pump wavelength and direction) and a certain orientation of the crystal (see subsection 3.2). One IR wavelength can be designed to phase match collinearly, whereas the successive wavelengths can satisfy non-collinear PM simultaneously, covering different sections (rings) in the object plane [98] (see Fig. 21(a)). Therefore, the entire object plane can be imaged in a single acquisition, however with a radial distribution of wavelengths in the 
image plane [98] (see Fig. 21(b)). In 1972, Lucy performed broadband IR imaging at $10 \mu \mathrm{m}$ with $30^{\circ} \mathrm{FoV}$, which corresponded to a $0.5 \mu \mathrm{m}$ spectral coverage [25]. Later on, researchers have demonstrated wide FoV by illuminating an object with broadband IR sources, such as supercontinuum sources [104], [162], heat sources (Globar) [125], and ASE sources [171][173].
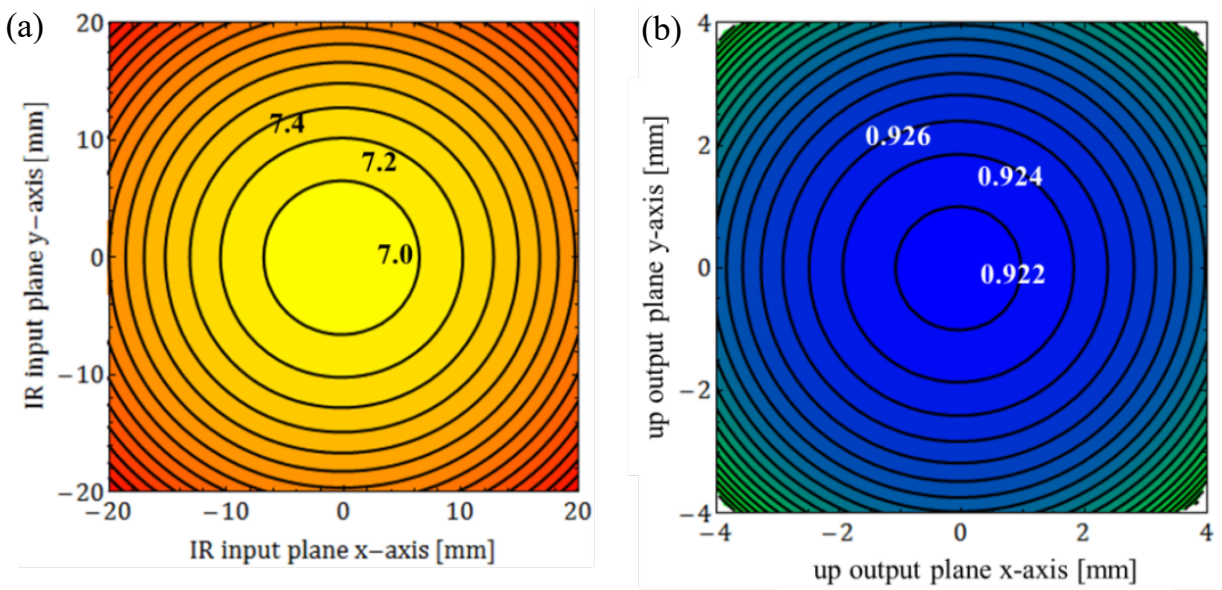

Fig. 21. Recalculated from [98]. Non-collinear phase matched wavelengths as a function of positions in (a) input IR plane using $f_{1}=50 \mathrm{~mm}$, (b) output image plane using $f_{2}=100 \mathrm{~mm}$, for an $\mathrm{AgGaS}_{2}$ crystal cut at $48^{\circ}$. The indexing numbers in (a) and (b) correspond to IR and upconverted wavelengths in $[\mu \mathrm{m}]$, respectively. The dark indexing circular lines are separated by (a) $0.2 \mu \mathrm{m}$ and (b) $0.002 \mu \mathrm{m}$.

As an example, Torregrosa et al. [173] demonstrated the variation in angular PM condition for different monochromatic IR and for a broadband ASE illumination (see Fig. 22). Correspondingly, the upconverted images of a USAF resolution target for monochromatic and ASE illumination operating at the telecom wavelength range are shown in Fig. 23(a), (b) [173].
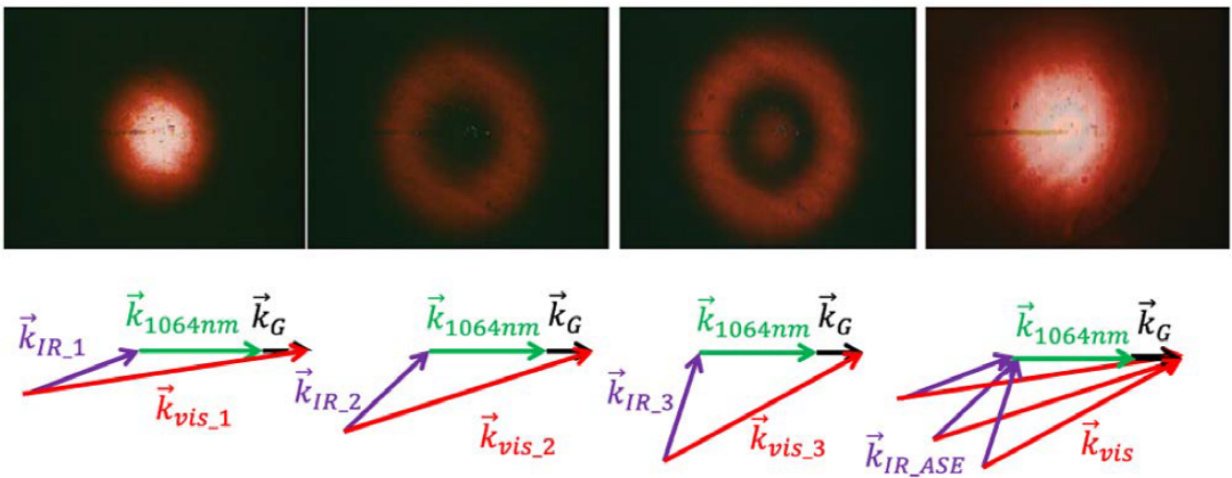

Fig. 22. Adapted with permission from [173] (C) The Optical Society. FoV of upconversion imaging in a PPLN based upconverter for IR illumination by (a) $1545 \mathrm{~nm}$, (b) $1546 \mathrm{~nm}$, (c) 1547 $\mathrm{nm}$ monochromatic laser, and (d) broadband ASE source (FWHM is $15 \mathrm{~nm}$ ).

Note that, in the case of a broadband IR illumination, the pump has to be monochromatic in order to get a one-to-one correspondence between the $\lambda_{\mathrm{IR}}$ and the $\lambda_{\text {up. Imaging using such }}$ techniques also reduces the efficiency of the conversion process as only a fraction of IR light at $\lambda_{\mathrm{IR}}$, corresponding to a certain angle $\left(\psi_{\mathrm{IR}}\right)$ and bandwidth gets upconverted due to requirement of PM. Furthermore, since the wavelength scaling, $\lambda_{\mathrm{up}} / \lambda_{\mathrm{IR}}$ is not linear, the upconverted images experience a radially dependent spatial magnification introducing distortion in the 
image, which increases for larger angles and larger spectral acceptance bandwidths (further discussion in sub-section 5.6).
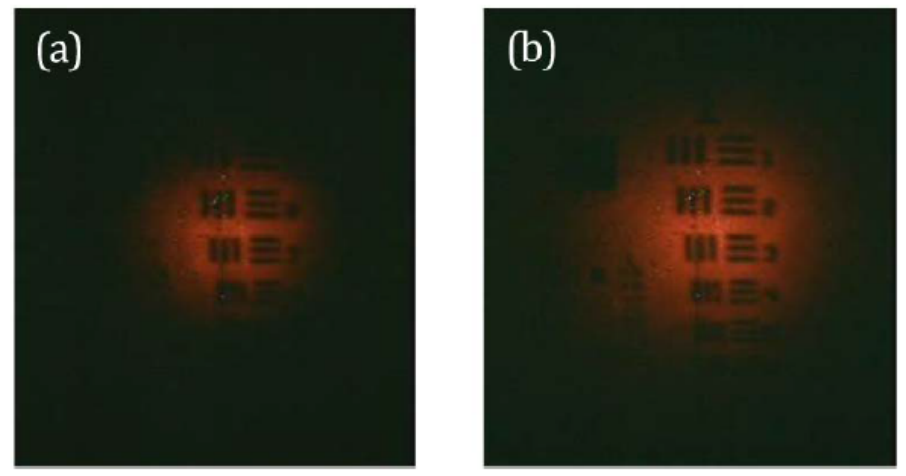

Fig. 23. Adapted with permission from [173] (C) The Optical Society. Upconverted image captured at $\sim 631 \mathrm{~nm}$ (upconverted wavelength) using a PPLN based upconverter when the object (standard USAF target) is illuminated by (a) a monochromatic laser and (b) an ASE source (15 $\mathrm{nm}$ bandwidth) at telecom wavelength.

\subsection{Angular resolution and number of resolvable elements}

In the case of upconversion in the Fourier plane (System-I, Fig. 5), the spherical wave originating from a point in the object plane corresponds to a plane wave inside the crystal. Here instead of a spatial resolution, one may consider angular resolution for convenience [71]. Using Fourier optics analysis [68] (section 2), the object resolution can be estimated by convolving a point object with the PSF of the imaging system. According to the standard Rayleigh criterion of resolution, two equally bright incoherent point objects can be resolved using a circular pupil when the center of the Airy pattern (PSF of the circular aperture) generated by one point object falls exactly on the first minima of the Airy pattern generated by the second point object (see Fig. 24(a)). The brightness at the center decreases by $\sim 26.3 \%$ relative to the maximum of the combined pattern. In case of an upconversion system with plane IR and plane pump wave, the finite crystal aperture sets the PSF. In reality, most of the bulk nonlinear crystal has a square aperture, and accordingly, the PSF is a 2-D sinc function (see Fig. 24(b)). Following the Rayleigh criterion, the angular resolution can be calculated at wavelength $\lambda$ as per Table 5 . In general, a strong pump (typically a laser) is used for the upconversion process. Therefore, in practice, the pump is a beam of finite size, which influences or even defines the PSF of the system. Typically, the pump beam waist diameter is smaller than the transverse dimension of the crystal, and the upconversion takes place in the region where the IR and the pump overlaps inside the crystal; hence, the pump beam size defines the spatial resolution (as also mentioned in section 2). Andrews have shown in [71] that to yield a diffraction-limited resolution, the pump needs to be diffraction limited too. Therefore, a single transverse mode pump (e.g. Gaussian pump beam) is desired to achieve the best resolution. The PSF associated with a Gaussian pump beam is also a Gaussian, and the angular resolution is defined by its far-field divergence angle. Considering three different definitions for calculating the beam diameter (FWHM, $1 / e$, and $1 / e^{2}$ intensity point), the angular resolutions can be calculated as listed in Table 5. Among them, the resolution calculated using the 1/e intensity point (the central dip of the combined PSF is $\sim 26 \%$ below the maximum as shown in Fig. 24(c)) gives the closest resemblance to the resolution defined by the standard Rayleigh definition for an Airy pattern. Further note that, a larger pump diameter improves the resolution [138]. 
(a)

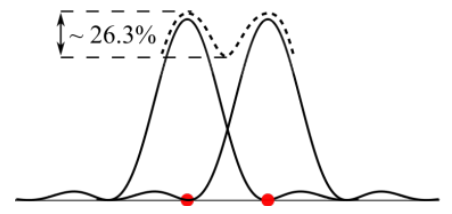

(b)

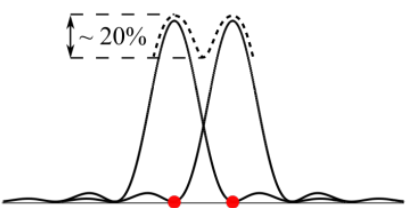

(c)

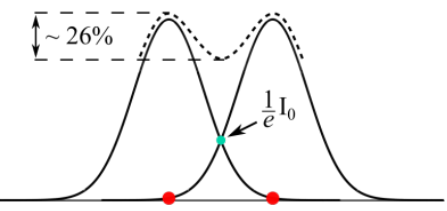

Fig. 24. Schematic one-dimensional intensity profile of the point-spread function for a (a) circular, (b) rectangular, and (c) Gaussian aperture. The Rayleigh criterion for angular resolution for the circular aperture is shown in (a) and similar analogy is made for (b) and (c). The red dots represent two equally bright incoherent point objects to be resolved.

Table 5. Angular Resolution for Different Apertures

\begin{tabular}{|c|c|c|c|c|c|c|}
\hline Aperture & $\begin{array}{c}\text { Circular } \\
\left(\text { diameter }=D_{\text {circle }}\right)\end{array}$ & $\begin{array}{c}\text { Square } \\
\left.\text { (width }=D_{\text {width }}\right)\end{array}$ & \multicolumn{4}{|c|}{ Gaussian $\left(\right.$ diameter $\left.=D_{\mathrm{FWHM}}, D_{1 / e}, \mathrm{D}_{1 / e}^{2}\right)$} \\
\hline $\begin{array}{c}\text { (Intensity } \\
\text { profile) }\end{array}$ & Airy pattern & $\operatorname{Sinc}^{2}$ function & \multicolumn{4}{|c|}{ Gaussian } \\
\hline \multirow{3}{*}{$\begin{array}{l}\text { Angular } \\
\text { Resolution }\end{array}$} & $1.22 \lambda$ & $\lambda$ & $\begin{array}{c}1 / e^{2} \\
\text { match point }\end{array}$ & \multicolumn{2}{|c|}{$\begin{array}{c}\text { FWHM } \\
\text { match point }\end{array}$} & $\begin{array}{c}1 / e \\
\text { match point }\end{array}$ \\
\hline & $D_{\text {circle }}$ & $D_{\text {width }}$ & $4 \lambda$ & $\sqrt{\ln (2)}$ & $4 \lambda$ & \multirow{2}{*}{$\sqrt{\ln (2)} \frac{4 \lambda}{\pi D_{1 / e}}$} \\
\hline & & & $\pi D_{1 / e^{2}}$ & 2 & $D_{\text {FWHM }}$ & \\
\hline
\end{tabular}

The total number of resolvable elements $\left(N_{\text {res }}\right)$ within a given FoV is equal to the spaceangular product of the upconversion system divided by the space-angular product of a single resolvable element and is given (for 2-D square aperture) by:

$$
N_{\text {res }}=\frac{\left(D_{\text {up }} \cdot \mathrm{FoV}\right)^{2}}{\left(D_{\mathrm{p}} \cdot \psi_{\text {div }}\right)^{2}},
$$

where $D_{\text {up }}$ is the diameter of the upconverted beam inside the crystal corresponding to the diameter of the pump beam $\left(D_{\mathrm{p}}\right)$, and $\psi_{\text {div }}$ is the far field divergence angle of the upconverted beam (which sets the angular resolution, see Table 5). Note that, $N_{\text {res }}$ can also be calculated in the object plane using Eq. (37) by changing "up" to "IR". Further note that, the beam divergence has less effect on the resolution for longer IR wavelength [174]. However, it is counter-acted by the fact that, longer IR wavelengths experience higher demagnification. For a diffraction limited upconverted beam (Gaussian beam), the space-angular product of a single resolvable element (using 1/e diameter) is given by

$$
D_{\mathrm{p}} \cdot \psi_{\mathrm{div}}=D_{\mathrm{up}} \cdot \psi_{\mathrm{div}}=\sqrt{\ln (2)} \frac{4 \lambda_{\mathrm{up}}}{\pi} .
$$

For System-I, the FoV can be calculated in terms of input parameters $D_{\mathrm{IR}}^{\mathrm{obj}}$ and $f_{1}$ ( $D_{\mathrm{IR}}^{\mathrm{obj}}$ is the diameter of the IR beam in the object plane which is dictated either by the full acceptance angle of the upconversion or the truncation of the optical system). $D_{\text {up }}$ is assumed to be the same as $D_{\mathrm{p}}$ (inside crystal). Thus, the space-angular product for the system is

$$
D_{\text {up }} \cdot \mathrm{FoV}=D_{\mathrm{p}} \cdot \frac{D_{\mathrm{IR}}^{\mathrm{obj}}}{f_{1}} \cdot \frac{\lambda_{\mathrm{up}}}{\lambda_{\mathrm{IR}}} .
$$

Therefore, the $N_{\text {res }}$ for this 2-D image can be calculated using Eqs. (37) - (39) as: 


$$
N_{\text {res }}=\frac{1}{\ln (2)}\left(\frac{\pi D_{\mathrm{p}} D_{\mathrm{IR}}^{\mathrm{obj}}}{4 f_{1} \lambda_{\mathrm{IR}}}\right)^{2} .
$$

The smallest resolvable features of an object can be further increased by increasing the system magnification factor $\left(f_{2} / f_{1}\right)$ [125], however, at the expense of a smaller FoV, i.e. the number of resolvable elements, $N_{\text {res }}$ remains unchanged. In the case of QPM crystals, the available vertical dimension is limited (typically $1 \mathrm{~mm}$ for PPLN) in comparison to the horizontal direction. Thus resolution (if the crystal size sets the PSF) also differs for these two directions [45], [95]. For a small FoV, going from a monochromatic to a polychromatic illumination, the resolution does not vary significantly [164], however with increasing FoV the image starts to appear blurred at the periphery of the FoV [175] as the spectral acceptance bandwidth increases significantly with the PM angle [69]. A list of reported resolution for upconversion imaging is given at the end of section 7. The above calculation is valid mainly for an incoherent illumination for the object. Calculation of resolution with a coherent object illumination is not straightforward as the resolution becomes a function of phase distribution in the object plane. Goodman [68] described this phase dependence using three cases of the twopoint resolution criterion, i.e., two neighboring point objects of equal amplitude are (i) in phase, (ii) in phase quadrature ( $\pi / 2$ phase difference), and (iii) out of phase. He concluded that case (ii) gives similar resolution as that of incoherent illumination, whereas case (i) and case (iii) result in poorer and better resolution, respectively, than the incoherent case. In a real scenario where the phase distribution in the object plane is complex or unknown, determining the exact resolution with coherent illumination would require a careful consideration similar to the twopoint resolution criterion described above.

\subsection{Imaging artifacts}

Upconversion imaging also suffers from different artifacts, some similar to the artifacts in direct imaging, and others originate from the upconversion process itself. The primary artifacts are originating from light diffraction, aberrations, speckle, distortion, and magnification error.

The diffraction of light from the object plane to the image plane mainly limits the resolution of the imaging. In a diffraction-limited geometry [68], the diffraction of the pump beam limits the spatial resolution. The aberrations arises from the imaging optics, for e.g., spherical aberration from lenses [176]. At upconverted wavelength $(<1 \mu \mathrm{m})$, high-quality aspheric lenses are available in the market (e.g. Thorlabs $\left.{ }^{\circledR}\right)$; however, there is limited choice for such lenses at the IR wavelengths, especially for the longer IR wavelengths $(>3 \mu \mathrm{m})$. This aberration increases for larger FoV. Often parabolic mirrors are used instead of lenses to eliminate this effect [104]. For broadband upconversion, an additional chromatic aberration arises from the chromatic error in lenses and the dispersion of the nonlinear crystal. This introduces a focusing error in the image plane. The wider spectral range one covers, the worse it gets. Further details on chromatic aberrations for different upconversion imaging setups can be found in [177]. As already mentioned previously, the aberration due to finite crystal length vanishes for System-I. The speckle pattern appears in coherent imaging. This can be eliminated by destroying the phase relation of the object signal, most often by using a rotating diffuser [69]. For monochromatic imaging, the image distortion can arise from imperfect optics, e.g. astigmatism from a non-planar beam combiner (pump and IR wave); see Fig. 25 [70]. The authors of [70] also pointed out that the blurring (a kind of distortion) in the upconverted image (Fig. 25(c), (d)) originated from the spatial filtering. The Gaussian pump, which acts as the Fourier filter for upconversion imaging in System-I, upconverts the higher spatial frequency components (section 2) less efficiently; hence the image lost its sharpness [70], [178]. A larger pump beam waist radius would correct this issue. Another kind of distortion occurs when the rotation of the nonlinear crystal is used to satisfy the phase matching condition over a large FoV for a given $\lambda_{\mathrm{IR}}$ [23], [125]. For different rotation angles, the angle-dependent refractive index (section 3) 
differs in two orthogonal planes, parallel and perpendicular to the crystal $\hat{c}$-axis. This introduces a magnification error in the image, however, this can be corrected by post-processing or using a "correction plate" [23]. Without rotation, this kind of magnification error has been observed for broadband upconversion imaging [164]. For broadband operation, another type of distortion namely Barrel (or Pincushion) arises. Under this distortion, any vertical or horizontal straight line in the object appears as convex (or concave) curve. In upconversion imaging, the ratio of the angular positions of a point object $\left(\psi_{\mathrm{IR}}\right)$ and the corresponding point image $\left(\psi_{\mathrm{up}}\right)$ is related to the ratio of their corresponding wavelengths, where $\left(\psi_{\text {up }} / \psi_{\mathrm{IR}}\right)=\left(\lambda_{\mathrm{up}} / \lambda_{\mathrm{IR}}\right)$. As the wavelength ratio is not linear, the angle and consequently the position ratio become nonlinear too. Therefore a perfect rectangular object experiences different wavelength dependent magnifications going from its center to the periphery [104], [162]. This type of distortion also increases for larger FoV. However, it can be corrected by image post-processing. It is also possible that, different acceptance bandwidths at different object locations can introduce different PSFs, which can blur the image inhomogeneously. This can be corrected by introducing an angular dependent PSF correction algorithm during image acquisition/postprocessing. Wavefront distortion due to spatial walk-off can also introduce minute distortions in wide FoV upconversion imaging, however, this can be avoided by choosing a thin crystal and proper PM condition.

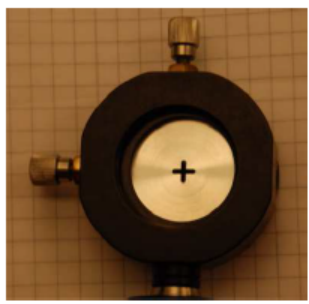

(a)

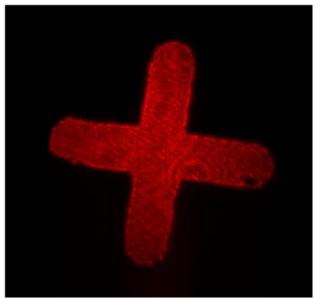

(b)

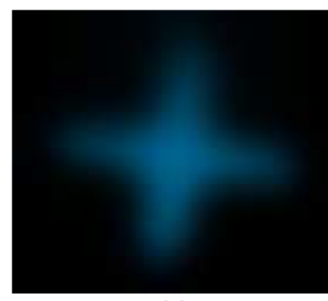

(c)

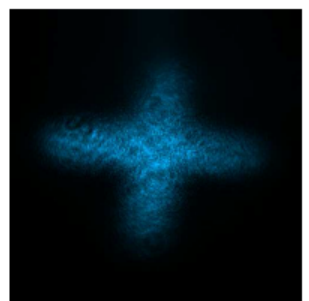

(d)

Fig. 25. Adapted from [70]. (a) Transmission mask used as an object. (b) Direct image of the $765 \mathrm{~nm}$ coherently illuminated mask. (c) Theoretically calculated light distribution in the image plane at the upconverted wavelength, $488 \mathrm{~nm}$. (d) Experimentally measured upconverted image.

\subsection{Multi-dimensional upconversion imaging}

Until now we discussed how upconversion can be used for 2-D spatial imaging. Different techniques have been demonstrated to improve the imaging FoV as well as the image quality. As the fundamental process of this imaging technique involves nonlinear (parametric) frequency conversion, the imaging is sensitive to both the temporal and spectral response of the object. Thus upconversion-based multi-dimensional imaging is possible where the other dimension could be wavelength, depth, phase, and/or time. Due to the space limit, only the hyperspectral imaging is reviewed in this section. Other multi-dimensional imaging options are briefly outlined in the "Conclusion and Outlook" section.

When the wavelength is used as a $3^{\text {rd }}$ dimension, it is widely known as hyperspectral imaging, which is one of the most successful applications of upconversion imaging and it has huge potential for the bio-chemical-medical application(s). The combination of hyperspectral imaging and the upconversion technique was already introduced in 1970's, when upconversion was used for chemical imaging of astronomical objects [27], [57], [106], [179]. However, those systems were limited to small FoV, low conversion efficiency, and narrowband upconversion. In recent years, hyperspectral imaging with wide FoV has been demonstrated including broadband upconversion [45], [47], [94], [125], [162], [180], [181]. For hyperspectral imaging, either a tunable narrowband (e.g., tunable laser, QCL, OPO) or broadband (e.g., Globar, supercontinuum, ASE) IR source is used as illumination. The ultimate goal is to get spectral information of each spatial element of the object. The imaging parameters, namely, FoV, spatial 
resolution, efficiency, and noise can be calculated similarly as described in previous sections. However, hyperspectral imaging is associated with an extra parameter, spectral resolution. For a tunable narrowband IR illumination, the spectral resolution is set by the wavelength tuning step and the linewidth of the illumination source. Whereas, for a broadband illumination, the spectral resolution is set by the acceptance bandwidth of the nonlinear conversion process [98], [106].

For tunable narrowband IR illumination, at each incoming $\lambda_{\mathrm{IR}}$, the PM condition is tuned either by rotating the crystal [69], [104], [125], by changing the temperature of the crystal [19], [45], [69], by raster-scanning the object [44], [180], or by tuning the pump wavelength [93]. For example, Junaid et al. [125] calculated the narrow annular FoV for each rotation angle of their nonlinear crystal $\left(\mathrm{AgGaS}_{2}\right)$ at a fixed $\lambda_{\mathrm{IR}}$ of $6.9 \mu \mathrm{m}$ (see Fig. 26(a)). By stitching the images corresponding to each rotational position (each PM condition), the monochromatic image at $6.9 \mu \mathrm{m}$ can be developed with a full FoV as discussed in sub-section 5.4.

With broadband IR illumination, as the spectral resolution is set by the spectral acceptance bandwidth of the upconversion process, a narrowband PM is desired to get a better spectral resolution. Tidemand-Lichtenberg et al. [98] demonstrated spectral resolution in the order of $10-18 \mathrm{~cm}^{-1}$ for detection in the $5-10 \mu \mathrm{m}$ IR range using non-collinear phase matching in an $\mathrm{AgGaS}_{2}$ crystal. They concluded that the spectral resolution deteriorates as angle increases. At larger PM angles (corresponding to longer wavelengths), the slope of the PM curve becomes steeper (see Fig. 12(b)), making the acceptance bandwidth wider and therefore, the resolution becomes poorer. This is a downside of broadband upconversion hyperspectral imaging, where spectral resolution varies with wavelength, and hence varies across the imaging FoV. Such non uniformity in spectral resolution sometimes creates spectral artifacts [69]. In the 1970's work on astronomical applications of upconversion, researchers confined their study to a small FoV (few degrees) and could achieve a high spectral resolution $(<2 \mathrm{~nm})$ in the $2-5 \mu \mathrm{m}$ IR range [106]. By using temperature [106] or angle [27] tuning, different IR wavelengths were phase matched over the same FoV, and hyperspectral images were formed by combining those set of images. In recent years, hyperspectral imaging using broadband IR illumination and wide FoV has been demonstrated [47], [94], [162], [181]. A schematic of the angular PM curve for broadband IR is shown in Fig. 26(b) [47], where the spectral response varies across the image position. By tuning the PM condition this spectral response can be varied across the image plane. After post-processing, monochromatic images can be formed by choosing one wavelength for the entire image plane (horizontal dotted line in Fig. 26(b)). By stitching all these monochromatic images, the hyperspectral data cube can be built. In another way, polychromatic information of different spatial position (vertical dashed line in Fig. 26(b)) can be extracted and then combined to form the hyperspectral data cube. Note that, unlike in the method using a tunable IR source, only one scanning (PM condition) is required in the scheme using broadband illumination. However, it does require a complicated post-processing stage to construct the hyperspectral data cube. By tuning the temperature [47], rotating the crystal [181], and tuning the pump wavelength [93], tuning of the PM curve has been demonstrated. Instead of tuning the PM condition, Kehlet et al. [94] suggested the translation of the object in the transverse plane to get spectral information from different parts of the object. The spectral resolution can further be increased by using a dispersive element (e.g. grating) at the output (upconverted) side before detecting it [112], [128]. Care should be taken to avoid over or under sampling. 

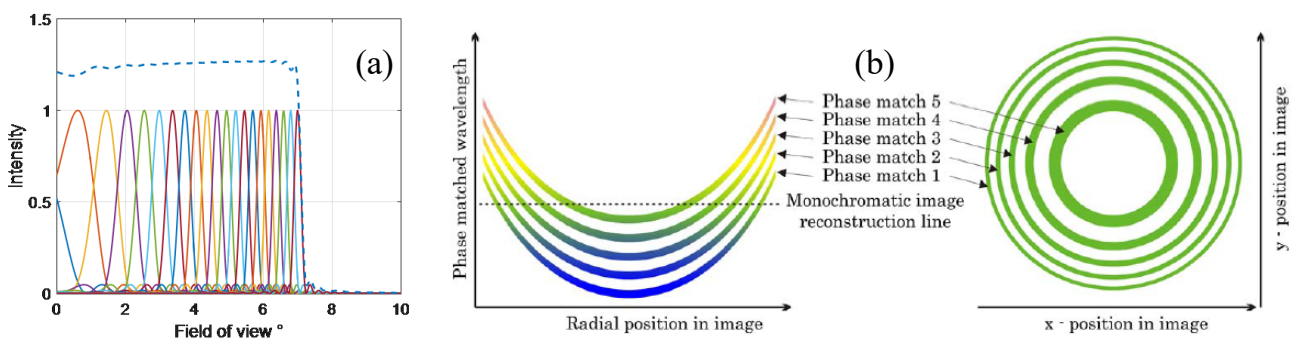

Fig. 26. (a) Tunable IR illumination: adapted from [125]. Simulated $\operatorname{sinc}^{2}$-curves describing the angular acceptance (1-D FoV) at $6.9 \mu \mathrm{m}$ for an $\mathrm{AgGaS}_{2}$ crystal (cut angle $48^{\circ}$ ) while rotating the crystal from $0^{\circ}$ to $10^{\circ}$ (different colors correspond to $0.5^{\circ}$ rotation step). Dashed line shows numerical sum of all intensity contribution within the full FoV. (b) Broadband IR illumination: adapted from [47]. Schematic PM curves are illustrated for a typical broadband upconverter. By tuning the PM condition, the PM curve can be tuned. A monochromatic image with full FoV can be reconstructed by post-processing the acquired data.

\section{Upconversion imaging applications}

In this section we discuss few example applications of upconversion imaging. The examples are based on bulk nonlinear crystals, and utilize either point detection combined with rasterscanning technique (to get wide FoV image) or 2-D array sensor based full-FoV imaging.

Researchers have also exploited waveguide configurations of the nonlinear material to maximize the three-wave interaction. It improves upconversion efficiency as well as the sensitivity of detection. Though such waveguide configuration is excellent for spectroscopic application and low-noise single-photon level detection [182], [183], they are not quite suitable for wide FoV imaging owing to the reduced aperture of the waveguide geometry ( $\mu \mathrm{m}$ scale). However, using more than one waveguides in parallel, techniques have been proposed demonstrating efficient coherent upconversion imaging of distant (faint) objects (i.e. astronomical applications) [91], [96]. Note that the waveguide based upconversion is outside the scope of this review, and related works on this topic can be found elsewhere.

\subsection{Raster-scan upconversion imaging}

In case of raster-scanning scheme, typically two configurations are considered. In one configuration (Fig. 27(a)) of the raster-scan imaging system, the object (which emits, transmits or reflects IR radiation) is fixed and the collection optics of the detection system performs a point-by-point scan of the object plane using beam-steering optics (e.g. dual-axis galvo mirrors). Alternatively, the object is mechanically scanned along the transverse plane such that different parts of the object are probed sequentially (Fig. 27(b)). Whereas, for 2-D array sensorbased imaging, typically a setup similar to System-I is used in combination with different scanning techniques mentioned in sub-section 5.4. 

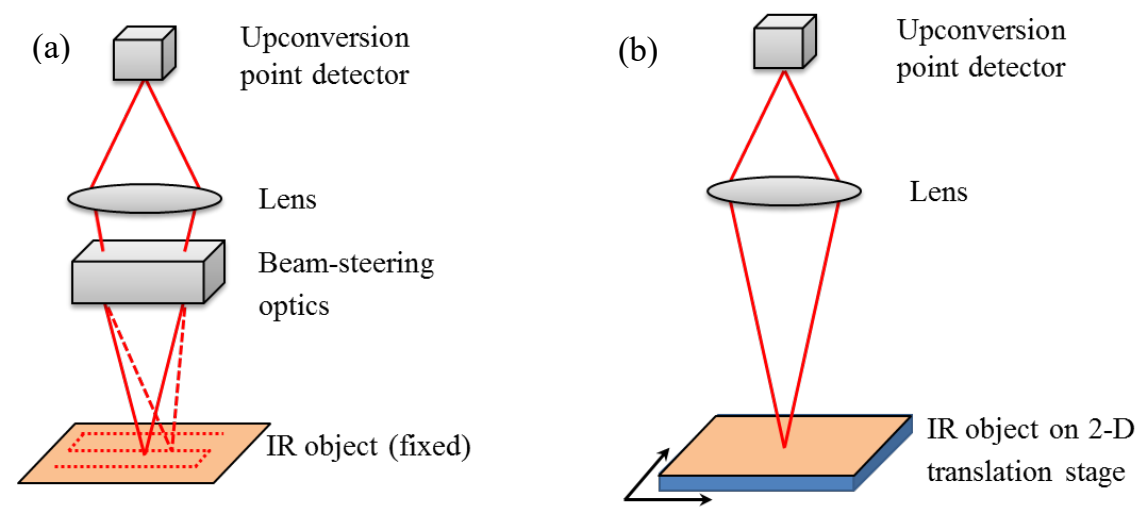

Fig. 27. Two common configurations of the raster-scan based upconversion imaging system: (a) fixed object and (b) scanned object configuration.

\section{1.a Gas leak detection}

In 1968, upconversion imaging employing the raster-scan technique was reported [184]. Imaki and Kobayashi demonstrated the use of a raster-scan imaging system with an upconversion point detector and scanning mirror to image at $3.39 \mu \mathrm{m}$ gas leaks of $\mathrm{CH}_{4}$ from a pipe [44], as shown in Fig. 28. The $3.39 \mu \mathrm{m}$ illumination source was alternately tuned to an on-resonance $\left(\lambda_{\text {on }}\right)$ and off-resonance ( $\left.\lambda_{\text {off }}\right)$ wavelength of a $\mathrm{CH}_{4}$ absorption line to obtain a differential image of the gas profile, as shown in Fig. 28(e). This is not a true hyperspectral imaging, however can be considered as two-color imaging instead. Furthermore, due to the use of a mirror mounted on a galvano-scanner to perform the point-by-point scan of the $3.39 \mu \mathrm{m}$ illumination beam, the method is limited to relatively low imaging frame rates.

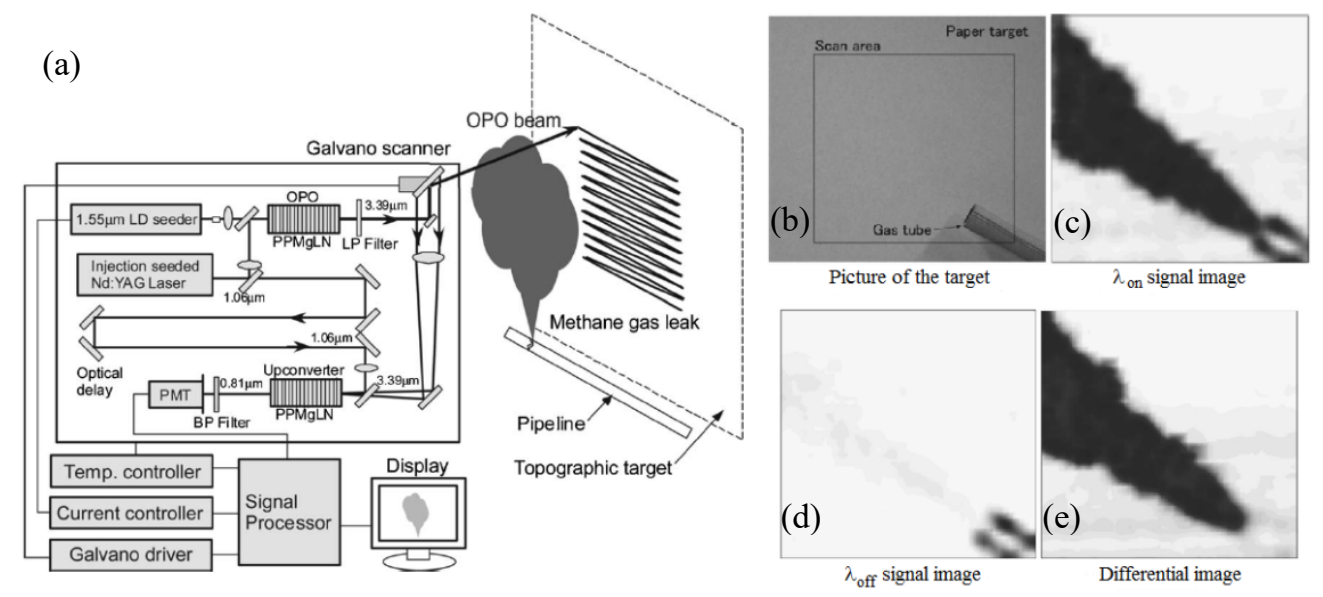

Fig. 28. Raster-scan based upconversion imaging of $\mathrm{CH}_{4}$ gas leak at $3.39 \mu \mathrm{m}$. (a) Schematic diagram of the experimental setup. (b) A photo of the scanned scene. Images obtained by the system when the illumination wavelength is (c) on-resonance and (d) off-resonance of an absorption line of $\mathrm{CH}_{4}$. (e) The differential image. Adapted with permission from [44] (C) The Optical Society.

\section{1.b Label-free breast cancer tissue diagnostics}

Recently, Tseng et al. [180], [185] exploited raster-scannig based wide FoV upconversion imaging using a bulk nonlinear crystal $\left(\mathrm{AgGaS}_{2}\right)$. They utilized the sample-scanning technique in a microscope setting to get high-resolution images in the $9-12 \mu \mathrm{m}$ IR range, using a tunable QCL for IR illumination (50 ns pulse). The acquisition speed must be optimized by choosing a 
two-stage scanning (i.e. tuning the IR wavelength and tuning the PM/raster-scan) in the proper order. A CW $1064 \mathrm{~nm}$ laser of $3 \mathrm{~W}$ power is used as single-pass pump for the upconversion process. The estimated spatial resolution is $\sim 35 \mu \mathrm{m}$ [180]. This upconversion microscope setup is then utilized for diagnostics of breast cancer tissue biopsies in the $9-12 \mu \mathrm{m}$ range, retrieving spectral signature of the phosphate and carbonate bands. For each QCL wavelength, a monochromatic upconverted image is developed pixel-by-pixel by raster-scanning the entire object (tissue sample of area $\sim \mathrm{mm}^{2}$ ) (see Fig. 29(a)). The process can then be repeated by sweeping the QCL wavelength to get spectrally encoded full FoV ( $\mathrm{mm}^{2}$ tissue) hyperspectral images (see Fig. 29(b)). The monochromatic full FoV image acquisition time is $\sim 30 \mathrm{sec}$, which is primarily limited by the speed of their microscope translation stage. The paper also compares the upconverted images with a traditional micro-FTIR system equipped with a liquid nitrogen cooled $\mathrm{HgCdTe}$-focal plane array.

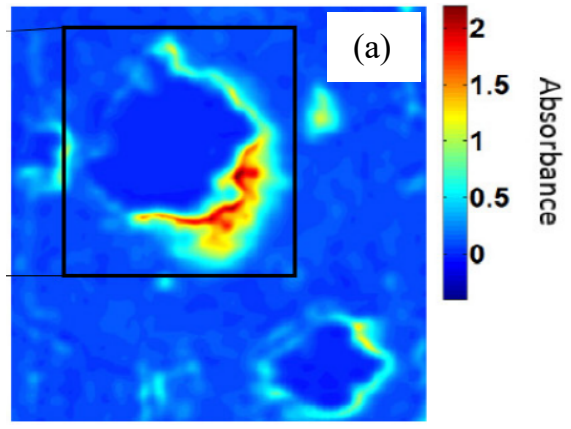

(b)
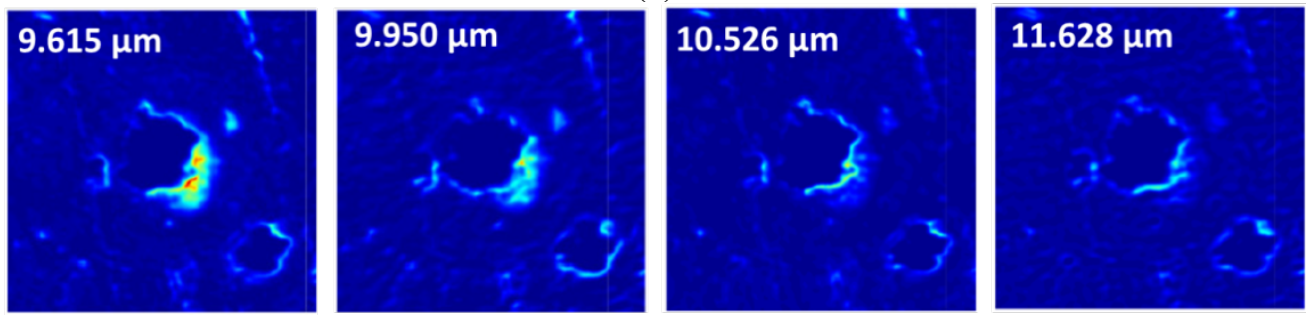

Fig. 29. (a-b) adapted from [180], raster-scanning based upconversion hyperspectral imaging of human breast cancer tissue $(1.3 \mathrm{~mm} \times 1.2 \mathrm{~mm})$ using a QCL illumination (a) at $\sim 9.8 \mu \mathrm{m}\left(\lambda_{\text {up }}=\right.$ $958.8 \mathrm{~nm}$ ), region marked by the black square is indicating the cancer infected area; and (b) at four other IR illumination wavelengths. The strength of absorbance (see color bar) changes for different wavelengths.

\section{1.c MIR optical coherence tomography for non-destructive testing}

In collaboration with researchers from other international universities, our group has recently demonstrated, for the first time, a high-resolution real-time optical coherence tomography (OCT) operating in the MIR spectral range (centered at $4 \mu \mathrm{m}$ ) [186]. The system employs the conventional scheme of spectral domain-OCT, however the "real-time" detection of MIR OCT signal is obtained by employing a broadband upconversion detection scheme. A fiber-based supercontinuum source emitting $0.9-4.7 \mu \mathrm{m}$ MIR light is used as illumination source. The light is first passed through a Michelson interferometer and focused onto a sample. Then the collected OCT signal (sample + reference) is relayed to a broadband upconversion detector for spectral conversion, where the OCT signal covering $3.6 \sim 4.7 \mu \mathrm{m}$ is directly upconverted to $820-865 \mathrm{~nm}$ NIR range employing simultaneous non-collinear upconversion of different spectral components (Fig. 30(a), also see sub-section 3.2). An intracavity upconversion scheme is used, where a diode $(3 \mathrm{~W}, 880 \mathrm{~nm})$ pumped $\mathrm{CW}$ solid state laser $\left(\mathrm{Nd}: \mathrm{YVO}_{4}\right)$ operating at $1064 \mathrm{~nm}$ is used as pump, and a $20 \mathrm{~mm}$ long PPLN crystal of poling period $23 \mu \mathrm{m}$ is used as 
the nonlinear crystal. The PPLN is placed inside the pump cavity delivering $30 \mathrm{~W}$ of intracavity power. Average upconversion quantum efficiency is $\sim 1 \%$ over the detection bandwidth. The upconverted spectra is detected using a silicon-spectrometer over 2286 pixels, which results in an average MIR sampling resolution of $0.46 \mathrm{~nm}$. The measured axial (depth) resolution is 8.6 $\mu \mathrm{m}$, which is mainly set by the spectral bandwidth of detection. The transverse imaging (OCT B-scan) is performed by scanning the sample plane (similar to Fig. 30(b)). The system works at A-scan rate of $0.33 \mathrm{kHz}$, which enables real-time B-scans at mm/s speed, and C-scans (3-D) at $\mathrm{mm}^{2} / \mathrm{min}$ rate. The researchers [186] demonstrated OCT imaging of different samples and assessed the suitability of MIR OCT in comparison to a state-of-the-art NIR OCT. One example is to image multi-layer ceramic samples (Fig. 30 (b)), which is imaged $(800 \times 730 \times 2048$ pixel volume) with high spatial resolution and good SNR within 36.5 min (Fig. 30(c)).
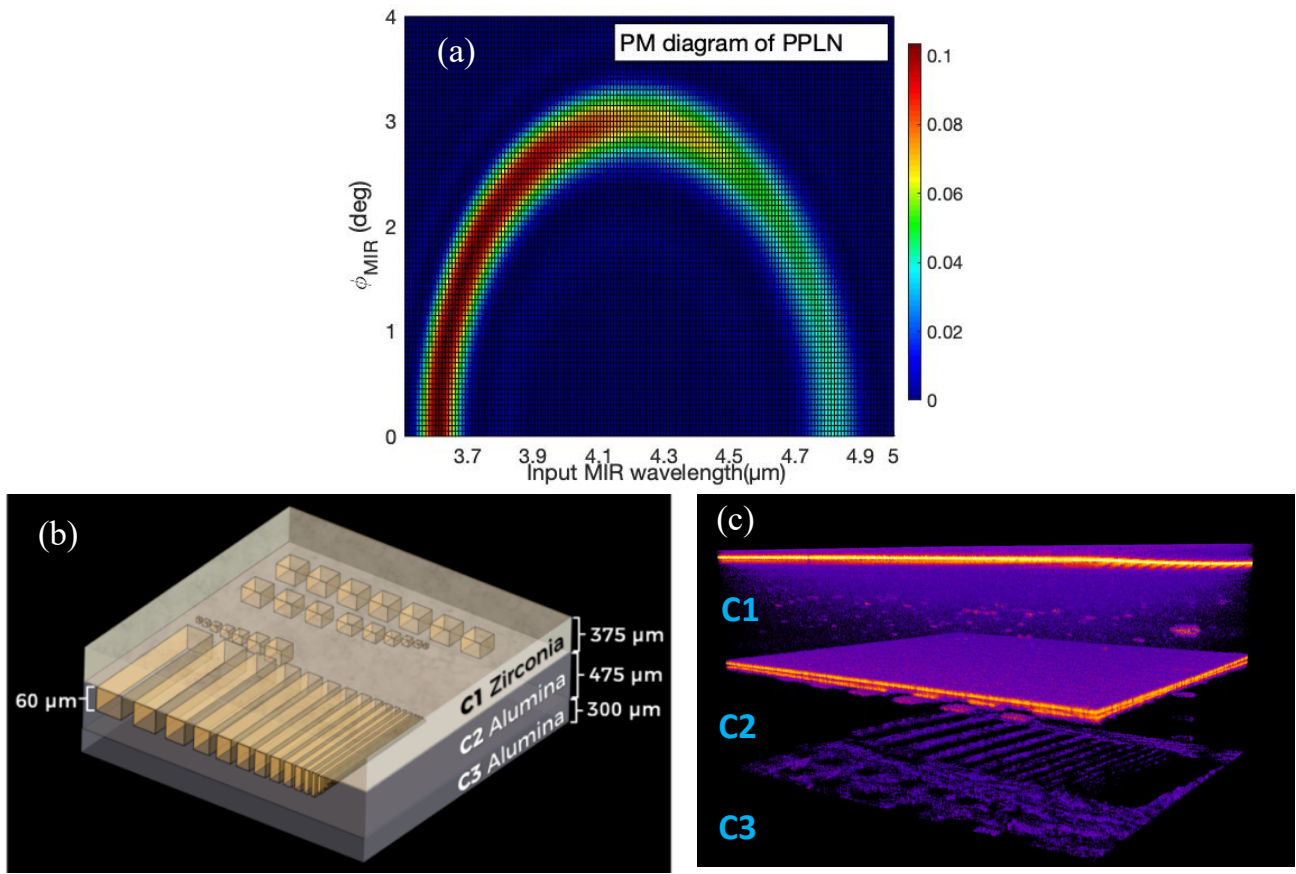

Fig. 30. (a) Calculated non-collinear phase-matching (PM) diagram of the $20 \mathrm{~mm}$ long PPLN of $\Lambda=23 \mu \mathrm{m}$ including pump depletion and linear absorption loss inside PPLN. The y-axis is the PM angle (internal between pump and MIR) for different phase matched MIR spectral components ( $\mathrm{x}$-axis). The color scheme represents normalized (to 0.1 ) conversion efficiency. (bc) adapted from [186]. (b) Schematic of a stack of ceramic sample under test, which consists of three layers of ceramic $(\mathrm{C} 1-\mathrm{C} 3)$ made of alumina and zirconia with patterned structures of variable size $(5-300 \mu \mathrm{m})$. (c) 3-D OCT image of the ceramic sample over a $5.6 \mathrm{~mm} \times 7.3 \mathrm{~mm}$ scanned transverse area.

\subsection{Video-rate wide FoV upconversion imaging}

Together with researchers from the Institute of Photonic Sciences in Barcelona and the University of Exeter, our group has recently demonstrated a novel upconversion imaging system for obtaining wide FoV MIR images $\left(N_{\text {res }}=64 \times 10^{3}\right)$ with a frame acquisition time of $2.5 \mathrm{~ms}$ [168]. The system employs an OPO emitting 20 ps laser pulses with a selectable wavelength in the $2.3-4 \mu \mathrm{m}$ range as a monochromatic illumination of an object plane. For every selected monochromatic MIR wavelength, a wide-FoV transmission image of the sample is obtained using a $4 f$ upconversion imaging setup (System-I) as shown in Fig. 31.

Upconversion occurs in a bulk lithium niobate nonlinear crystal held by a rotatable galvo scanner and centered in the Fourier plane of the $4 f$ imaging setup. High conversion efficiency 
as well as a high SNR is achieved by using an upconversion pump laser in the form of high peak power $1064 \mathrm{~nm}$ pulses that are in temporal synchronism with MIR signal pulses. In order to obtain wide-FoV upconverted images, the system implements an improved version of the nonlinear crystal rotation technique discussed in sub-section 5.4.

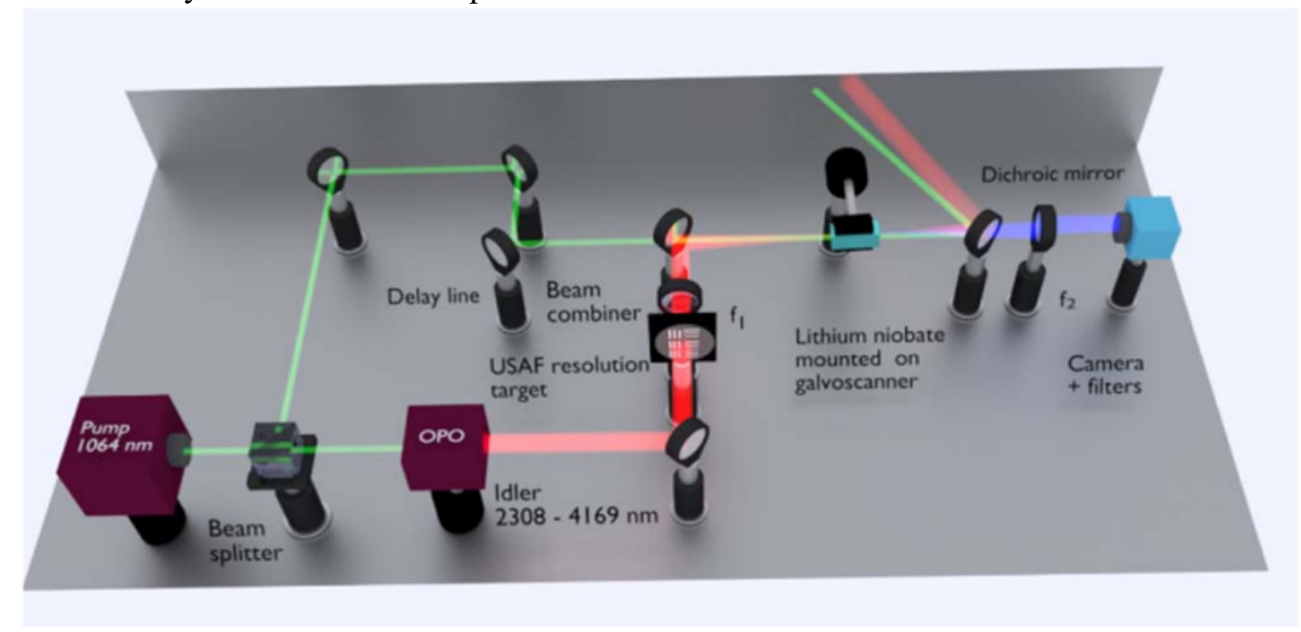

Fig. 31. Adapted from [168]. Setup for an upconversion-based imaging, where the idler beam from a picosecond OPO is used as an illumination source and a synchronized picosecond 1064 $\mathrm{nm}$ laser source is used as the upconversion pump source. The beams are spatially and temporally overlapped in the nonlinear crystal (lithium niobate) for efficient upconversion. The PM condition is scanned by rotating the crystal in synchronism with the integration time of a siliconbased CCD camera. Lenses, $f_{1}(50 \mathrm{~mm})$ and $f_{2}(50 \mathrm{~mm}$ or $100 \mathrm{~mm}$, depending on sample used), comprise the $4 f$ imaging setup. Filters (shortpass $950 \mathrm{~nm}$, longpass $700 \mathrm{~nm}$ ) block residual unwanted stray light.

\section{2.a Label-free esophageal cancer tissue diagnostics}

In [168], thin unstained esophageal biopsy samples on a $\mathrm{CaF}_{2}$ substrate were placed in the object plane (replacing the resolution target) of the upconversion imaging system in Fig. 31. As the OPO illumination source and the upconversion imaging are tunable in operation wavelength, MIR hyperspectral imaging (HSI) of esophageal cancer tissue samples were obtained and compared with images obtained by a state-of-the-art FTIR system (see Fig. 32).
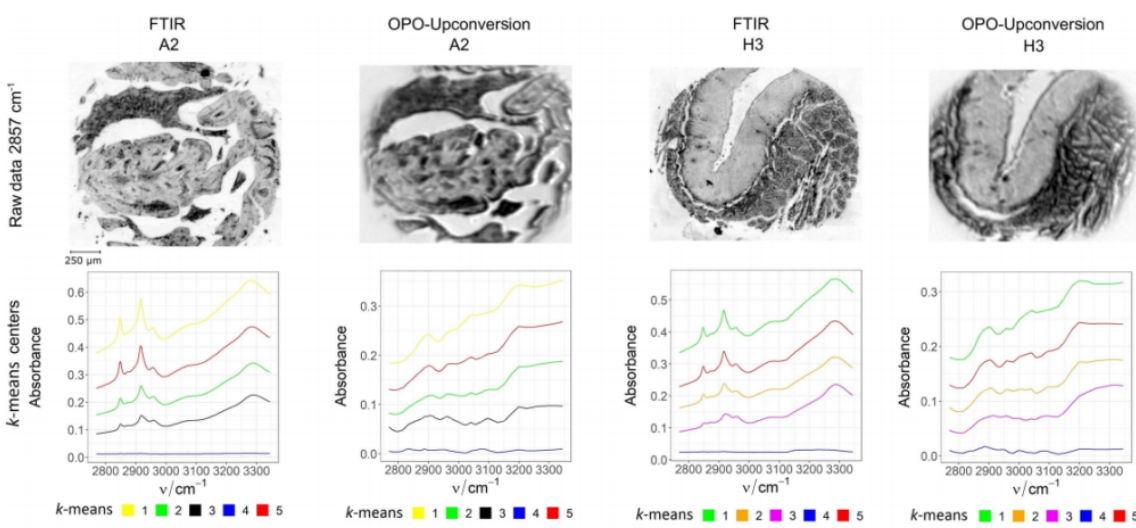

Fig. 32. Adapted from [168]. (Top row) Images of the tissue sample acquired using FTIR and upconversion. (Bottom row) Spectral analysis of the cancerous and healthy tissue sample, based on upconversion imaging and FTIR. 
Figure 32 shows generally a good agreement between the tissue images obtained by the upconversion system and the FTIR system. Although the latter still currently provides better spatial and spectral resolution, our results show the potential of a MIR OPO-upconversion imaging system in providing hyperspectral data from human tissue biopsy sections for preliminary, unsupervised clustering of different tissue types, with a performance similar to FTIR imaging. Further improvement of the upconversion imaging system could potentially lead to fast computer-assisted cancer biopsy screening, as exemplified by the monochromatic MIR image acquisition just within a few milliseconds.

\section{2.b Real-time gas leak profiling}

We also demonstrated the application of the video-rate wide FoV upconversion imaging system for obtaining real-time 2-D profile of a butane gas leak. Using the setup in Fig. 31 with the OPO illumination source set at a fixed wavelength $\lambda_{\mathrm{IR}}=3.37 \mu \mathrm{m}$, we captured wide FoV images of a transmission-type USAF resolution target while a butane gas leaking from a lighter is placed in front of the object plane - see Fig. 33 and the supplementary material (Visualization 3 ) found in [168]. Note that in the visualization (i.e. video clip), the profile of the butane gas leak, which appears as dark shade due to a strong absorption line of butane at $3.37 \mu \mathrm{m}$, can be tracked at a high frame rate and much better spatial resolution than that obtained with the rasterscan approach described in sub-section 6.1.a. Although our demonstration of real-time gas leak imaging is in transmission mode, the system can be configured in reflection geometry. Finally, the ability to tune the wavelength of illumination and detection allows for spectroscopic imaging for identification of gas mixture with potentially improved specificity.

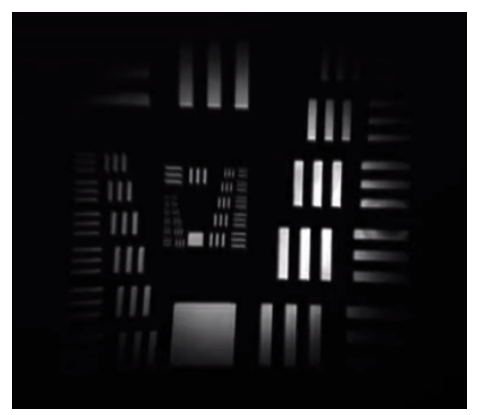

Fig. 33. Adapted from the supplementary material of [168]. Upconverted image of a USAF resolution target at $3.37 \mu \mathrm{m}$ while rotating the crystal with respect to c-axis by $1^{\circ}$ and in synchronism with the camera integration time of $2.5 \mathrm{~ms}$.

\section{Other $\chi^{(2)}$ parametric processes relevant for IR imaging}

Apart from the upconversion (sum frequency generation) scheme (Fig. 34(a)), difference frequency generation (DFG)/optical parametric amplifier (OPA) (Fig. 34(b)) based parametric processes have also been exploited for converting IR images to the VIS/NIR range or amplifying IR images for subsequent detection. In the case of an OPA (or DFG), an intense pump (at $\lambda_{\mathrm{p}}$ ) is passed through a $\chi^{(2)}$ material together with a IR wave (at $\left.\lambda_{\mathrm{IR}}\right)$ acting as a seed, resulting in the creation of idler wave (at $\lambda_{\mathrm{id}}$ ) and enhancement of IR wave. In this process, the pump is downconverted to two lower energy photons, one at $\lambda_{\text {id }}$ and the other at $\lambda_{\mathrm{IR}}$, where $\lambda_{\mathrm{IR}}$ $>\lambda_{\text {id }}>\lambda_{\mathrm{p}}$. In this way, a weak seed IR signal can be amplified. The gain of such an amplifier depends on the power of the input pump. Although DFG and OPA are both second order nonlinear parametric processes, their phase matching criteria are different from the upconversion process, even if the same pump and nonlinear crystal are chosen. Laferriere et al. [187] mentioned that "This amplification of the image makes downconversion ideal for lownoise optical-image amplifiers". However, unlike upconversion imaging, the amplified OPA image is detected at the illumination wavelength. This makes the OPA imaging difficult at longer IR wavelengths. To date, upconversion based imaging has received much attention in 
comparison to downconversion based imaging. Nonetheless, the first OPA based monochromatic imaging with wide FoV was proposed by Devaux et al. [42]. Note that the DFG and OPA based imaging are outside the scope of the present review, however the reader may consider these review articles [139], [188] for more details. The recent advances in DFG/OPA based imaging using different nonlinear crystals can also be found in [135], [189]-[193]. Researchers have also utilized second harmonic generation (SHG) for IR imaging. Recent demonstration on SHG-based IR imaging can be found for $1 \mu \mathrm{m}$ [194]-[196], $1.342 \mu \mathrm{m}$ [197], and $2 \mu \mathrm{m}[198]$ illumination wavelengths.
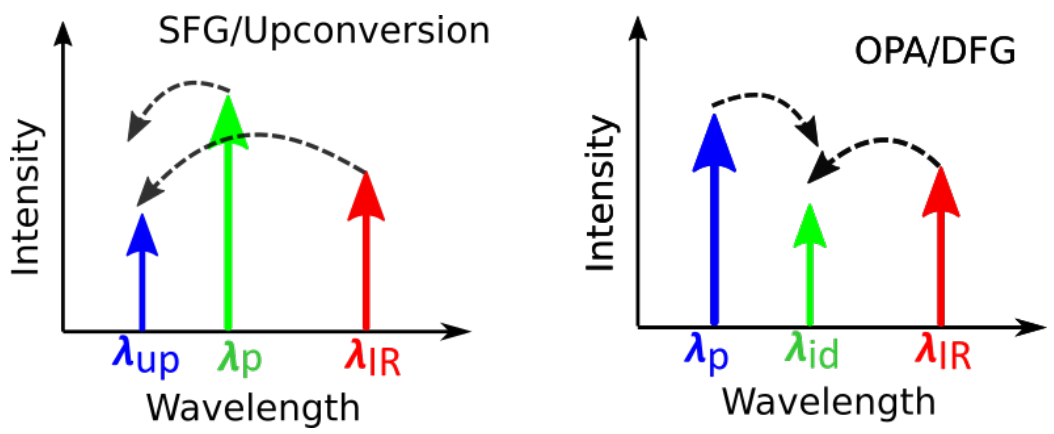

Fig. 34. Spectral diagram of second order parametric processes (a) SFG/Upconversion and (b) OPA/DFG in a $\chi^{(2)}$ medium. The dashed arrows indicate the direction of energy flow.

In Table 6, we report the published results in parametric upconversion imaging using bulk nonlinear crystals, starting from its first demonstration in 1968 by Midwinter to date. The list shows the progress in the field and indicates how the choice of experimental parameters has changed over the years.

Table 6. Reported Results on Parametric Upconversion Spatial Imaging using Bulk Crystal

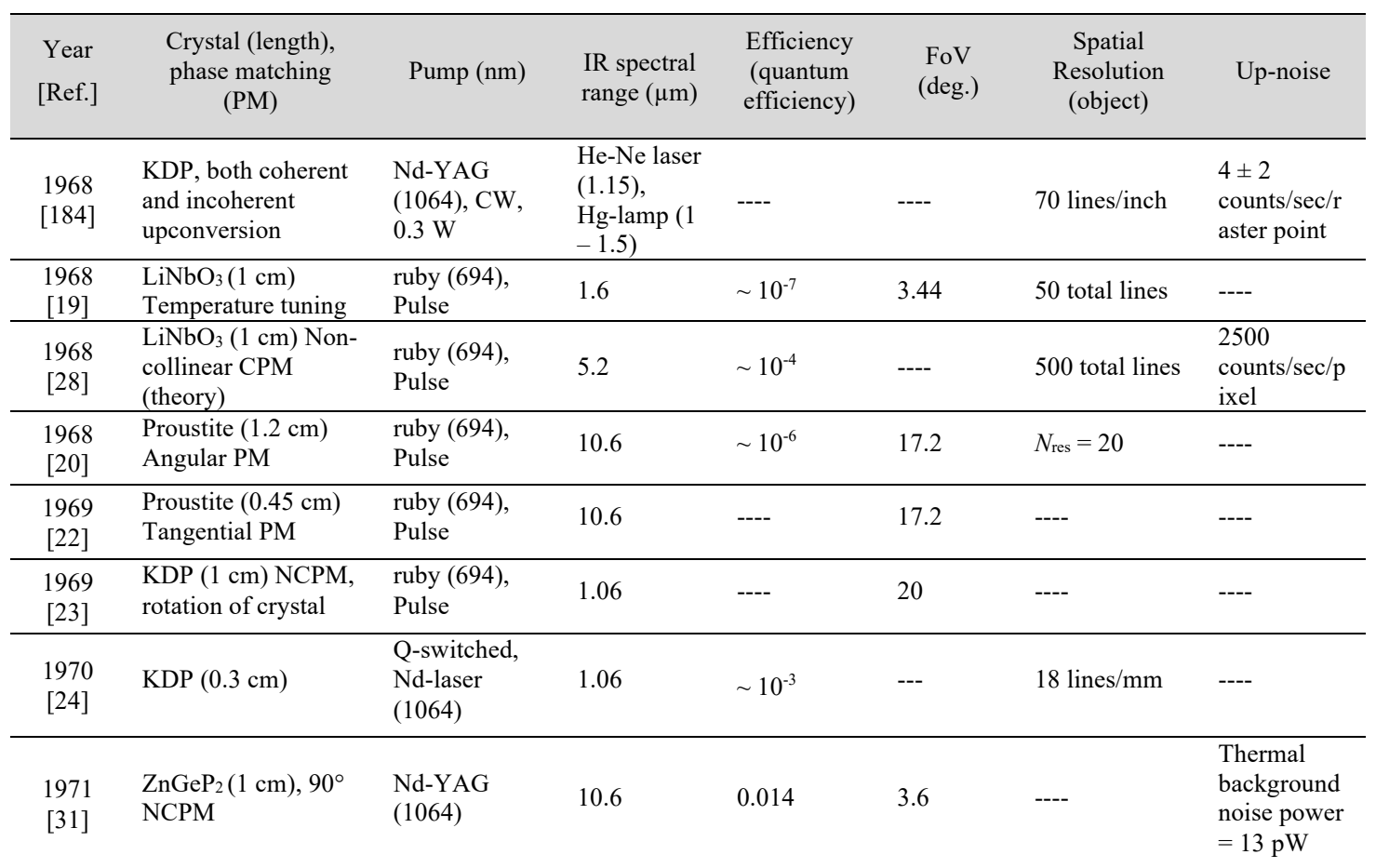




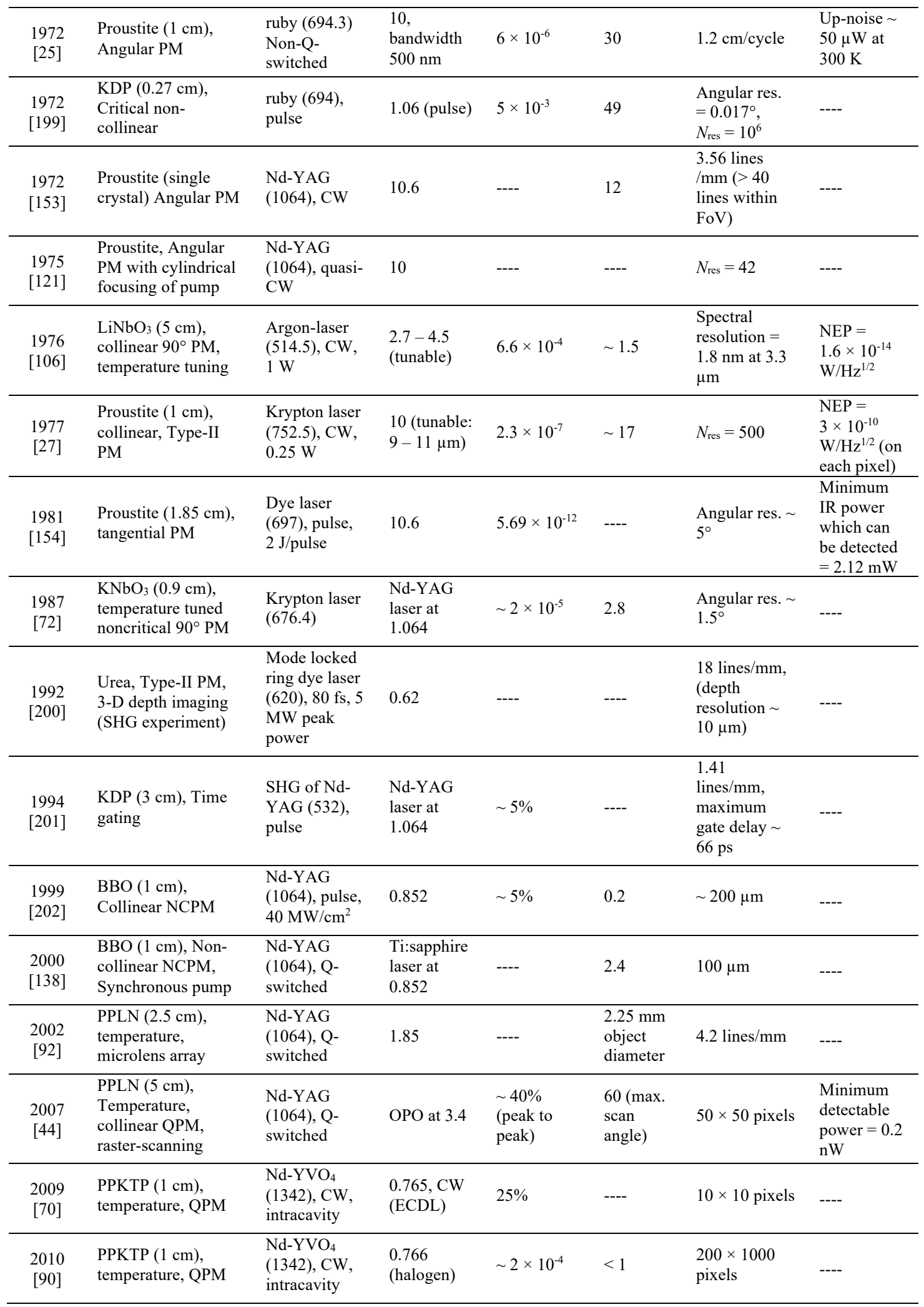




\begin{tabular}{|c|c|c|c|c|c|c|c|}
\hline $\begin{array}{l}2012 \\
{[45]}\end{array}$ & $\begin{array}{l}\text { PPLN }(2 \mathrm{~cm}) \text {, } \\
\text { temperature tuning, } \\
\text { QPM }\end{array}$ & 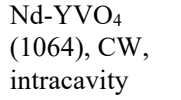 & $\begin{array}{l}3 \text { (tunable } \\
\text { from } 2.85- \\
5 \mu \mathrm{m})\end{array}$ & $\begin{array}{l}\sim 20 \% \\
\text { (polarized, } \\
\text { collinear) }\end{array}$ & --- & $\begin{array}{l}200 \times 100 \\
\text { pixels }\end{array}$ & $\begin{array}{l}0.2 \text { photons/ } \\
\text { sec/pixel }\end{array}$ \\
\hline $\begin{array}{l}2014 \\
{[204]}\end{array}$ & $\begin{array}{l}\text { DAST, Collinear, } \\
\text { difference frequency } \\
\text { generation }\end{array}$ & $\begin{array}{l}\text { KTP-OPO } \\
(1292)\end{array}$ & $19.3 \mathrm{THz}$ & ---- & $\begin{array}{l}40 \mathrm{~mm} \\
\text { object } \\
\text { diameter }\end{array}$ & $\sim 3 \mathrm{~mm}$ & $\begin{array}{l}\text { Signal to } \\
\text { noise ratio } \\
(\mathrm{SNR})=30\end{array}$ \\
\hline $\begin{array}{l}2015 \\
{[94]}\end{array}$ & $\begin{array}{l}\text { PPLN }(2 \mathrm{~cm}), \text { QPM, } \\
\text { non-collinear, } \\
\text { Hyperspectral }\end{array}$ & $\begin{array}{l}\mathrm{Nd}^{-\mathrm{YVO}_{4}} \\
(1064), \mathrm{CW}, \\
\text { intracavity }\end{array}$ & $3.24-3.41$ & ---- & $\begin{array}{l}1 \mathrm{~cm}^{2} \\
\text { object } \\
\text { area }\end{array}$ & $\begin{array}{l}231 \mu \mathrm{m}(\mathrm{PSF}) \\
\text { spectral res. } \sim \\
9-30 \mathrm{~nm}\end{array}$ & ---- \\
\hline $\begin{array}{l}2015 \\
{[173]}\end{array}$ & $\begin{array}{l}\text { PPLN }(0.5 \mathrm{~cm}) \\
\text { QPM, non-collinear }\end{array}$ & $\begin{array}{l}\text { Nd-YVO } \\
(1064), \mathrm{CW}, \\
\text { intracavity }\end{array}$ & $\begin{array}{l}1.55, \\
\text { FWHM = } \\
15 \mathrm{~nm}\end{array}$ & $\sim 10^{-4}$ & $>1.6$ & 4 lines/mm & $\begin{array}{l}\text { Limited by } \\
\text { CCD dark } \\
\text { noise }\end{array}$ \\
\hline $\begin{array}{l}2015 \\
{[178]}\end{array}$ & $\begin{array}{l}\text { PPLN }(5 \mathrm{~cm}), \mathrm{QPM}, \\
\text { Phase imaging }\end{array}$ & $\begin{array}{l}\text { Yb-doped } \\
\text { laser (1036) }\end{array}$ & 1.558 & $\sim 68 \%$ & ---- & ---- & $\begin{array}{l}3.8 \times 10^{3} \\
\text { counts } / \mathrm{sec}\end{array}$ \\
\hline $\begin{array}{l}2016 \\
{[104]}\end{array}$ & $\begin{array}{l}\mathrm{LiNbO}_{3}(1 \mathrm{~cm}), \\
\text { angle tuning, } \\
\text { synchronous }\end{array}$ & $\begin{array}{l}\text { Er-doped } \\
\text { fiber laser } \\
(1550), \text { pulse }\end{array}$ & $\begin{array}{l}\text { Superconti- } \\
\text { nuum, } 1.8- \\
2.6\end{array}$ & $\begin{array}{l}\sim 1.2 \times 10^{-5} \\
\text { (collinear) }\end{array}$ & $\begin{array}{l}\sim 1.9 \mathrm{~mm} \\
\text { image } \\
\text { diameter }\end{array}$ & $\sim 55 \mu \mathrm{m}$ & ---- \\
\hline $\begin{array}{l}2017 \\
{[206]}\end{array}$ & $\begin{array}{l}\text { PPLN }(4 \mathrm{~cm}), Q P M \text {, } \\
\text { temperature tuning }\end{array}$ & $\begin{array}{l}\mathrm{Nd}^{-\mathrm{YVO}_{4}} \\
(1064), \mathrm{CW}, \\
\text { intracavity }\end{array}$ & $\begin{array}{l}\text { Heat lamp } \\
2.9-3.5\end{array}$ & $\begin{array}{l}\sim 28 \% \\
\text { (collinear, } \\
\text { polarized) }\end{array}$ & ---- & $\begin{array}{l}N_{\text {res }} \sim 120 \times \\
70\end{array}$ & $\begin{array}{l}0.57 \\
\text { photons } / \mathrm{sec} / \\
\text { pixel }\end{array}$ \\
\hline $\begin{array}{l}2017 \\
{[162]}\end{array}$ & $\begin{array}{l}\mathrm{AgGaS}_{2}(1 \mathrm{~cm}), \\
\text { angle tuning, } \\
\text { Hyperspectral }\end{array}$ & $\begin{array}{l}\text { Er-doped } \\
\text { fiber laser } \\
(1550), n \mathrm{~s}\end{array}$ & $\begin{array}{l}\text { Superconti- } \\
\text { nuum, } 2- \\
4.5\end{array}$ & $\begin{array}{l}\sim 1.0 \times 10^{-4} \\
\text { (collinear) }\end{array}$ & $\begin{array}{l}14.9 \mathrm{~mm} \\
\text { object } \\
\text { diameter }\end{array}$ & $\begin{array}{l}\sim 79 \mu \mathrm{m}(12.7 \\
\text { lines } / \mathrm{mm}) \\
N_{\text {res }} \sim 32400\end{array}$ & ---- \\
\hline $\begin{array}{l}2017 \\
{[97]}\end{array}$ & $\begin{array}{l}\mathrm{LiNbO}_{3}(1 \mathrm{~cm}), \\
\text { angle tuning, } \\
\text { synchronous }\end{array}$ & $\begin{array}{l}\text { fiber laser } \\
(1550), p \mathrm{~s}\end{array}$ & $\begin{array}{l}\text { Er-Tm } \\
\text { fiber laser } \\
1.877\end{array}$ & ---- & $\begin{array}{l}2.63 \mathrm{~mm}^{2} \\
\text { object } \\
\text { area }\end{array}$ & $\begin{array}{l}\sim 23 \mu \mathrm{m} \\
N_{\mathrm{res}} \sim 5000\end{array}$ & ---- \\
\hline $\begin{array}{l}2018 \\
{[125]}\end{array}$ & $\begin{array}{l}\operatorname{AgGaS}_{2}(1 \mathrm{~cm}), \\
\text { angle tuning, } \\
\text { hyperspectral }\end{array}$ & $\begin{array}{l}\mathrm{Nd}-\mathrm{YVO}_{4} \\
(1064), \mathrm{CW}, \\
1.7 \mathrm{~W}\end{array}$ & $\begin{array}{l}\text { Globar, } \\
6-8\end{array}$ & ---- & $\begin{array}{l}\sim 21 \mathrm{~mm} \\
\text { object } \\
\text { diameter }\end{array}$ & $\begin{array}{l}\sim 35 \mu \mathrm{m}, \\
N_{\text {res }} \sim 4400, \\
\text { spectral res. }> \\
8 \mathrm{~cm}^{-1}\end{array}$ & ---- \\
\hline $\begin{array}{l}2018 \\
{[170]}\end{array}$ & $\begin{array}{l}\text { PPLN }(0.5 \mathrm{~cm}) \text {, } \\
\text { non-collinear QPM, } \\
\text { thermal gradient }\end{array}$ & $\begin{array}{l}\text { Nd-YAG } \\
(1064)\end{array}$ & 1.554 & $\begin{array}{l}0.25 \\
\text { (normalized } \\
\text { to the case } \\
\Delta \mathrm{T}=0^{\circ} \mathrm{C} \text { ) }\end{array}$ & $\begin{array}{l}\sim 8 \\
(\text { for } \Delta \mathrm{T}= \\
\left.50^{\circ} \mathrm{C}\right)\end{array}$ & ---- & ---- \\
\hline
\end{tabular}




\begin{tabular}{|c|c|c|c|c|c|c|c|}
\hline $\begin{array}{l}2018 \\
{[67]}\end{array}$ & $\begin{array}{l}\mathrm{AgGaS}_{2}(1 \mathrm{~cm}), \\
\text { collinear, angle } \\
\text { tuning }\end{array}$ & $\begin{array}{l}\mathrm{Nd}-\mathrm{YVO}_{4} \\
(1064), \mathrm{CW}, \\
3 \mathrm{~W}\end{array}$ & $\begin{array}{l}\text { QCL, } \\
\text { tunable } 9.4 \\
-12, \text { pulse }\end{array}$ & $\sim 1.0 \times 10^{-2}$ & $\sim 0.4$ & ---- & $\begin{array}{l}\text { SNR }>10 \\
\text { (for single } \\
\text { pulse) }\end{array}$ \\
\hline $\begin{array}{l}2018 \\
{[180]}\end{array}$ & $\begin{array}{l}\operatorname{AgGaS}_{2}(1 \mathrm{~cm}), \\
\text { Raster-scanning, } \\
\text { Hyperspectral }\end{array}$ & $\begin{array}{l}\mathrm{Nd}-\mathrm{YVO}_{4} \\
(1064), \mathrm{CW}, \\
3 \mathrm{~W}\end{array}$ & $\begin{array}{l}\text { QCL, } \\
\text { tunable } 9.4 \\
-12, \text { pulse } \\
\end{array}$ & $\sim 1.0 \times 10^{-2}$ & $\begin{array}{l}\sim 1.3 \times \\
1.2 \mathrm{~mm}^{2} \\
\text { object } \\
\text { area } \\
\end{array}$ & $\begin{array}{l}<35 \mu \mathrm{m}, \\
\text { spectral res. } ~ \\
1 \mathrm{~cm}^{-1}\end{array}$ & --- \\
\hline $\begin{array}{l}2019 \\
{[186]}\end{array}$ & $\begin{array}{l}\text { PPLN }(2 \mathrm{~cm}), \text { non- } \\
\text { collinear QPM, } \\
\text { Object scanning in } \\
\text { 3-D OCT imaging }\end{array}$ & $\begin{array}{l}\mathrm{Nd}^{-} \mathrm{YVO}_{4} \\
(1064), \mathrm{CW}, \\
\text { intracavity }\end{array}$ & $\begin{array}{l}\text { Superconti- } \\
\text { nuum, } 3.5- \\
4.7,1 \mathrm{MHz}\end{array}$ & $\sim 1 \%$ & $\begin{array}{l}\sim 1 \mathrm{~cm}^{2} \\
\text { object } \\
\text { area }\end{array}$ & $\sim 15 \mu \mathrm{m}$ & ---- \\
\hline $\begin{array}{l}2019 \\
{[207]}\end{array}$ & $\begin{array}{l}\mathrm{LiNbO}_{3}(1 \mathrm{~cm}), \\
\text { electronically } \\
\text { synchronous }\end{array}$ & $\begin{array}{l}\text { MOPA } \\
(1064), \text { pulse, } \\
1.6 \mathrm{~ns}\end{array}$ & $\begin{array}{l}\text { Superconti- } \\
\text { nuum, } 2- \\
4.2,1.6 \mathrm{~ns} \text {, } \\
40 \mathrm{kHz}\end{array}$ & $1 \%$ & $\begin{array}{l}13 \mathrm{~mm} \\
\text { object } \\
\text { diameter }\end{array}$ & $<78.8 \mu \mathrm{m}$ & $\begin{array}{l}\mathrm{SNR} \text { is } \sim 1 \\
\text { (integration } \\
\text { time } 0.5 \mathrm{~ms} \text { ) }\end{array}$ \\
\hline $\begin{array}{l}2019 \\
{[168]}\end{array}$ & $\begin{array}{l}\mathrm{LiNbO}_{3}(1 \mathrm{~cm}), \\
\text { tangential PM, } \\
\text { rotation of crystal }\end{array}$ & $\begin{array}{l}\text { Yb- fiber } \\
\text { laser }(1064), \\
\text { pulse, } 20 \mathrm{ps}, \\
80 \mathrm{MHz}\end{array}$ & $\begin{array}{l}\text { Optical } \\
\text { parametric } \\
\text { oscillator, } \\
\text { tunable } 2.3 \\
-4.1, \text { pulse }\end{array}$ & $\begin{array}{l}0.002 \% \text { for } \\
\text { each pixel } \\
\text { over full } \\
\text { FoV }\end{array}$ & $\begin{array}{l}10 \mathrm{~mm} \\
\text { object } \\
\text { diameter }\end{array}$ & $\begin{array}{l}\sim 35 \mu \mathrm{m}\left(N_{\text {res }}\right. \\
=64,000) \text { at } \\
3.1 \mu \mathrm{m}\end{array}$ & $\begin{array}{l}\mathrm{SNR}=424 \\
\text { at the center } \\
\text { of image }\end{array}$ \\
\hline $\begin{array}{l}2019 \\
{[208]}\end{array}$ & $\begin{array}{l}\mathrm{LiNbO}_{3}(1 \mathrm{~cm}), \\
\text { birefringent PM, } \\
\text { polychromatic, } \\
\text { rotation of crystal }\end{array}$ & $\begin{array}{l}\text { Modelocked } \\
\text { Ti:sapphire } \\
(804), 100 \mathrm{fs} \text {, } \\
80 \mathrm{MHz}\end{array}$ & $\begin{array}{l}\text { Optical } \\
\text { parametric } \\
\text { oscillator, } \\
\text { tunable } 2.7 \\
-4,200 \mathrm{fs}\end{array}$ & $\sim 10^{-5}$ & $\begin{array}{l}\sim 20 \text { at } \\
2.851 \\
\mu \mathrm{m} .\end{array}$ & $\begin{array}{l}\sim 56 \mu \mathrm{m} \text { at } \\
3.206 \mu \mathrm{m}\end{array}$ & ---- \\
\hline
\end{tabular}

\section{Conclusion and Outlook}

In this paper, we have extensively reviewed the infrared imaging technique based on novel $\chi^{(2)}$ parametric frequency upconversion process, starting back from its first experimental demonstration in 1968 to date. We have first introduced the $\chi^{(2)}$ nonlinear (upconversion) imaging based on generalized three dimensional Green function approach. In the theory we have shown how the classical diffraction theory can be translated to the nonlinear $\chi^{(2)}$ case. We have established far-field solutions similar to the Fraunhofer approximation and have established imaging criteria using usual lens system(s). Mathematical formulae for calculating upconversion efficiency and imaging resolution have been derived. We discussed two types of upconversion imaging setup, namely System-I (upconversion in Fourier plane) and System-II (upconversion in image plane) and established their properties. Then we have discussed the details on phase matching (PM) conditions starting from collinear interaction of three waves to non-collinear interaction and tunability of the PM parameters, which are important for designing an upconversion imaging with an expanded field-of-view (FoV). Noise properties in upconversion detector is also studied and compared with state-of-the-art direct MIR detector ( $\mathrm{HgCdTe})$ through reported results. Details on manipulation of FoV, angular resolution, various choices of nonlinear material, pumping conditions, presence of any artifacts are discussed for design purpose of an upconversion imaging system. Upconversion can be used for multidimensional imaging, and as an example, we have discussed and extensively reviewed the hyperspectral upconversion imaging. We have devoted a section for showcasing recent applications of parametric upconversion imaging exploiting either raster-scanning scheme or 2-D detector sensor-based scheme.

In a multi-dimensional upconversion imaging, the third dimension can also be the temporal characteristics, depth, or even phase of the object. From late 1970's, parametric upconversion is used for time gating application by utilising the fact that the upconversion takes place only when both the pump and the IR fields temporally overlap inside the $\chi^{(2)}$ nonlinear medium [209]. This upconversion time gating is primarily explored for studying transient phenomena like fluorescence characterization [210]-[212] and ultrafast time-resolved spectroscopy [54], [124], 
[165], [213], and been used for detecting objects embedded in scattering media [201], [214] and even for temporal characterization at single-photon level [215], [216]. There are only a few reported results, where time gating is combined with spatial imaging for characterizing transient phenomena of a 2-D object [136], [217]. Further study in this direction will open up a vast research and application field, where one can envision wide FoV and spectrally resolved transient infrared imaging [198]. Upconversion can be used to measure depth with $\mu \mathrm{m}$ scale resolution in a scattering media [186], [200], for optical coherence tomography (OCT) based 3-D imaging in the mid-infrared with real-time operation [186]; for construction of infrared to terahertz hologram [30], [189], [218], [219], where the image is reconstructed at a wavelength different from the reading light. Recently, researchers have utilized the phase invariant properties of parametric upconversion to demonstrate phase imaging [178], [194], [220], frequency conversion of structured light [221] and image manipulation by controlling the phase matching condition [222].

The inherent advantage of upconversion imaging systems over those that rely on commercially available cooled mid-infrared detectors is that they do not require sophisticated cooling, are capable of delivering enhanced sensitivity, and can provide higher number of resolvable elements. Thus, upconversion imaging in the mid-infrared is suitable for various imaging applications from low photon and quantum imaging, high resolution 2-D imaging in a microscope setup to multi-dimensional imaging. To date, the noise measurement is mainly done for collinear upconversion and in the shorter infrared wavelengths $(<4 \mu \mathrm{m})$. The conversion efficiency and noise of an upconverter are also primarily measured for collinear interactions and over a small FoV. Further research is needed for exploration beyond these limits. Upconversion of longer mid-infrared wavelengths is more challenging owing to limited options for suitable nonlinear materials as well as the inherently lower quantum conversion efficiency due to wider separation between the pump and the infrared signal wavelengths.

New applications [132], [136], [180], [222], [223], cross-disciplinary research, and technological improvements would propel this field of research and will potentially result in many more interesting scientific outcomes in the coming years.

\section{Funding, acknowledgments, and disclosures}

\subsection{Funding}

Innovation Fund Denmark, ShapeOCT (4107-00011A) and E11504 MIRILUS (Eurostars).

\subsection{Acknowledgments}

The authors would like to acknowledge Ashik A. S. of DTU Fotonik for providing a calculated figure.

\subsection{Disclosures}

The authors declare that there are no conflicts of interest related to this article.

\section{References}

[1] A. B. Seddon, B. Napier, I. Lindsay, S. Lamrini, P. M. Moselund, and N. Stone, "Midinfrared Spectroscopy/Bioimaging: Moving toward MIR optical biopsy," Laser Focus World, vol. 52, no. 2, pp. 50-53, 2016.

[2] Y. J. Kaufman and L. A. Remer, "Detection of forests using mid-IR reflectance: an application for aerosol studies," IEEE Trans. Geosci. Remote Sens., vol. 32, no. 3, pp. 672-683, May 1994.

[3] P. Colarusso, L. H. Kidder, I. W. Levin, J. C. Fraser, J. F. Arens, and E. N. Lewis, "Infrared Spectroscopic Imaging: From Planetary to Cellular Systems," Appl. Spectrosc., vol. 52, no. 3, pp. 106A-120A, Mar. 1998. 
[4] A. Sijan, "Development of military lasers for optical countermeasures in the mid-IR," in Technologies for Optical Countermeasures VI, 2009, vol. 7483, p. 748304.

[5] "Boson Compact LWIR Thermal Camera Core | FLIR Systems." [Online]. Available: https://www.flir.com/products/boson/. [Accessed: 18-Oct-2018].

[6] D. Ostrower, "Optical Thermal Imaging - replacing microbolometer technology and achieving universal deployment," III-VS Rev., vol. 19, no. 6, pp. 24-27, Aug. 2006.

[7] A. Rogalski, "Infrared detectors: an overview," Infrared Phys. Technol., vol. 43, no. 3, pp. 187-210, Jun. 2002.

[8] J.-S. Fang et al., "Path-dependent human identification using a pyroelectric infrared sensor and Fresnel lens arrays," Opt. Express, vol. 14, no. 2, pp. 609-624, Jan. 2006.

[9] "VIGO System S.A.," https://www.vigo.com.pl. .

[10] T. H. Maiman, "Stimulated Optical Radiation in Ruby," Nature, vol. 187, no. 4736, pp. 493-494, Aug. 1960.

[11] P. A. Franken, A. E. Hill, C. W. Peters, and G. Weinreich, "Generation of Optical Harmonics," Phys. Rev. Lett., vol. 7, no. 4, pp. 118-119, Aug. 1961.

[12] J. A. Armstrong, N. Bloembergen, J. Ducuing, and P. S. Pershan, "Interactions between Light Waves in a Nonlinear Dielectric," Phys. Rev., vol. 127, no. 6, pp. 1918-1939, Sep. 1962.

[13] G. P. Agrawal, Nonlinear Fiber Optics, 4th ed. Elsevier, 2006.

[14] X. Liu, C.-H. Yan, and J. A. Capobianco, "Photon upconversion nanomaterials," Chem. Soc. Rev., vol. 44, no. 6, pp. 1299-1301, Mar. 2015.

[15] M. Haase and H. Schäfer, "Upconverting Nanoparticles," Angew. Chem. Int. Ed., vol. 50, no. 26, pp. 5808-5829, Jun. 2011.

[16] F. Auzel, "Upconversion and Anti-Stokes Processes with $\mathrm{f}$ and d Ions in Solids," Chem. Rev., vol. 104, no. 1, pp. 139-174, Jan. 2004.

[17] A. W. Smith and N. Braslau, "Optical Mixing of Coherent and Incoherent Light [Letter to the Editor]," IBM J. Res. Dev., vol. 6, no. 3, pp. 361-362, Jul. 1962.

[18] J. E. Midwinter and J. Warner, "Up-Conversion of Near Infrared to Visible Radiation in Lithium-meta-Niobate," J. Appl. Phys., vol. 38, no. 2, pp. 519-523, Feb. 1967.

[19] J. E. Midwinter, "Image conversion from $1.6 \mu$ to the visible in lithium niobate," Appl. Phys. Lett., vol. 12, no. 3, pp. 68-70, 02-01 1968.

[20] J. Warner, "SPATIAL RESOLUTION MEASUREMENTS IN UP-CONVERSION FROM 10.6 m TO THE VISIBLE," Appl. Phys. Lett., vol. 13, no. 10, pp. 360-362, Nov. 1968.

[21] A. H. Firester, "Parametric Image Conversion: Part I," J. Appl. Phys., vol. 40, no. 12, pp. 4842-4849, Nov. 1969.

[22] J. Warner, "Phase-matching for optical up-conversion with maximum angular aperture - theory and practice," Opto-Electron., vol. 1, no. 1, pp. 25-28, Feb. 1969.

[23] R. Andrews, "Wide angular aperture image up-conversion," IEEE J. Quantum Electron., vol. 5, no. 11, pp. 548-550, Nov. 1969.

[24] E. S. Voronin, M. I. Divlekeev, Y. A. Il'Inskii, and V. S. Solomatin, "Image conversion from the infrared to the visible by nonlinear optics methods," vol. 31, no. 1, pp. 51-59, Jul. 1970.

[25] R. F. Lucy, "Infrared to Visible Parametric Upconversion," Appl. Opt., vol. 11, no. 6, pp. 1329-1336, Jun. 1972.

[26] D. Y. Tseng, "Synchronously pulsed ir up-conversion," Appl. Phys. Lett., vol. 21, no. 8, pp. 382-384, Oct. 1972.

[27] R. W. Boyd and C. H. Townes, "An infrared upconverter for astronomical imaging," Appl. Phys. Lett., vol. 31, no. 7, pp. 440-442, Oct. 1977.

[28] J. Midwinter, "Parametric infrared image converters," IEEE J. Quantum Electron., vol. 4, no. 11, pp. 716-720, Nov. 1968. 
[29] A. H. Firester, "Upconversion: Imaging with Planar and Spherical Pump Beams," Appl. Opt., vol. 9, no. 10, pp. 2266-2268, Oct. 1970.

[30] A. H. Firester, "Image Upconversion: Part III," J. Appl. Phys., vol. 41, no. 2, pp. $703-$ 709, Feb. 1970.

[31] G. D. Boyd, W. B. Gandrud, and E. Buehler, "PHASE-MATCHED UP CONVERSION OF 10.6- $\mu$ RADIATION IN ZnGeP2," Appl. Phys. Lett., vol. 18, no. 10, pp. 446-448, May 1971.

[32] J. Falk and W. B. Tiffany, "Theory of parametric upconversion of thermal images," $J$. Appl. Phys., vol. 43, no. 9, pp. 3762-3769, Sep. 1972.

[33] É. S. Voronin and V. L. Strizhevskiī, "Parametric up-conversion of infrared radiation and its applications," Sov. Phys. Uspekhi, vol. 22, no. 1, p. 26, 1979.

[34] J. A. Giordmaine, "Mixing of Light Beams in Crystals," Phys. Rev. Lett., vol. 8, no. 1, pp. 19-20, Jan. 1962.

[35] P. D. Maker, R. W. Terhune, M. Nisenoff, and C. M. Savage, "Effects of Dispersion and Focusing on the Production of Optical Harmonics," Phys. Rev. Lett., vol. 8, no. 1, pp. 21-22, Jan. 1962.

[36] W. S. Boyle and G. E. Smith, "Charge coupled semiconductor devices," Bell Syst. Tech. J., vol. 49, no. 4, pp. 587-593, Apr. 1970.

[37] J. Falk and J. M. Yarborough, "Detection of Room-Temperature Blackbody Radiation by Parametric Upconversion," Appl. Phys. Lett., vol. 19, no. 3, pp. 68-70, Aug. 1971.

[38] A. Szilagyi, A. Hordvik, and H. Schlossberg, "A quasi-phase-matching technique for efficient optical mixing and frequency doubling," J. Appl. Phys., vol. 47, no. 5, pp. 2025-2032, May 1976.

[39] M. S. Piltch, C. D. Cantrell, and R. C. Sze, "Infrared second-harmonic generation in nonbirefringent cadmium telluride," J. Appl. Phys., vol. 47, no. 8, pp. 3514-3517, Aug. 1976.

[40] J. Falk and Y. See, "Internal Cw Parametric Upconversion," Appl. Phys. Lett., vol. 32, no. 2, pp. 100-101, 1978.

[41] J. Faist, F. Capasso, D. L. Sivco, C. Sirtori, A. L. Hutchinson, and A. Y. Cho, "Quantum Cascade Laser," Science, vol. 264, no. 5158, pp. 553-556, Apr. 1994.

[42] F. Devaux et al., "Picosecond Parametric Amplification of a Monochromatic Image," Nonlinear Opt., vol. 11, pp. 25-37, 1995.

[43] F. Devaux and E. Lantz, "Transfer function of spatial frequencies in parametric image amplification: experimental analysis and application to picosecond spatial filtering," Opt. Commun., vol. 114, no. 3, pp. 295-300, Feb. 1995.

[44] M. Imaki and T. Kobayashi, "Infrared frequency upconverter for high-sensitivity imaging of gas plumes," Opt. Lett., vol. 32, no. 13, pp. 1923-1925, Jul. 2007.

[45] J. S. Dam, P. Tidemand-Lichtenberg, and C. Pedersen, "Room-temperature mid-infrared single-photon spectral imaging," Nat. Photonics, vol. 6, no. 11, pp. 788-793, Sep. 2012.

[46] C. R. Petersen et al., "Mid-infrared supercontinuum covering the 1.4-13.3 $\mu \mathrm{m}$ molecular fingerprint region using ultra-high NA chalcogenide step-index fibre," Nat. Photonics, vol. 8, no. 11, pp. 830-834, Nov. 2014.

[47] L. M. Kehlet, P. Tidemand-Lichtenberg, J. S. Dam, and C. Pedersen, "Infrared upconversion hyperspectral imaging," Opt. Lett., vol. 40, no. 6, pp. 938-941, Mar. 2015.

[48] T. Williams, Thermal Imaging Cameras: Characteristics and Performance, 1st ed. CRC Press, 2009.

[49] F. Marsili et al., "Detecting single infrared photons with 93\% system efficiency," Nat. Photonics, vol. 7, no. 3, pp. 210-214, Mar. 2013.

[50] K. S. Krishnan, J. S. Ostrem, and E. A. Stappaerts, "Infrared Imaging Using Nonlinear Optical Upconversion,” Opt. Eng., vol. 17, no. 2, p. 172108, Apr. 1978. 
[51] L. Hogstedt et al., "Low-noise mid-IR upconversion detector for improved IRdegenerate four-wave mixing gas sensing," Opt. Lett., vol. 39, no. 18, pp. 5321-5324, Sep. 2014.

[52] R. L. Pedersen, D. Hot, and Z. Li, "Comparison of an InSb Detector and Upconversion Detector for Infrared Polarization Spectroscopy," Appl. Spectrosc., vol. 72, no. 5, pp. 793-797, May 2018.

[53] M. D. Eisaman, J. Fan, A. Migdall, and S. V. Polyakov, "Invited Review Article: Singlephoton sources and detectors," Rev. Sci. Instrum., vol. 82, no. 7, p. 071101, Jul. 2011.

[54] J. Knorr, P. Rudolf, and P. Nuernberger, "A comparative study on chirped-pulse upconversion and direct multichannel MCT detection," Opt. Express, vol. 21, no. 25, pp. 30693-30706, Dec. 2013.

[55] J. Warner, "Parametric up-conversion from the infra-red," Opto-Electron., vol. 3, no. 1, pp. 37-48, Feb. 1971.

[56] A. F. Milton, "Upconversion-a Systems View," Appl. Opt., vol. 11, no. 10, pp. 23112330, Oct. 1972.

[57] R. W. Boyd, "Infrared Upconversion for Astronomy," Opt. Eng., vol. 16, no. 6, p. 166563, Dec. 1977.

[58] R. L. Byer, "Nonlinear Optical Phenomena and Materials," Annu. Rev. Mater. Sci., vol. 4, no. 1, pp. 147-190, 1974.

[59] M. M. Abbas, T. Kostiuk, and K. W. Ogilvie, "Infrared upconversion for astronomical applications," Appl. Opt., vol. 15, no. 4, pp. 961-970, 04-01 1976.

[60] R. W. Boyd, Nonlinear Optics: Third Edition. ScienceDirect, 2008.

[61] R. L. Sutherland, D. G. McLean, and S. Kirkpatrick, Handbook of nonlinear optics, 2nd ed., rev.Expanded. New York: Marcel Dekker, 2003.

[62] K. Rottwitt and P. Tidemand-Lichtenberg, Nonlinear Optics: Principles and Applications, 1st ed. CRC Press, 2014.

[63] G. D. Boyd and D. A. Kleinman, "Parametric Interaction of Focused Gaussian Light Beams,” J. Appl. Phys., vol. 39, no. 8, pp. 3597-3639, Jul. 1968.

[64] K. F. Lee and H. B. Ahmad, "A simple model for the calculation of the walk-off angle in uniaxial crystal," Opt. Commun., vol. 104, no. 1, pp. 111-117, Dec. 1993.

[65] B. E. A. Saleh and M. C. Teich, Fundamentals of Photonics, 3rd ed., vol. 2. John Wiley \& Sons, Inc., 2019.

[66] J. M. Blackledge, "Chapter 5 - Green Functions," in Digital Image Processing, J. M. Blackledge, Ed. Woodhead Publishing, 2005, pp. 113-152.

[67] Y.-P. Tseng, C. Pedersen, and P. Tidemand-Lichtenberg, "Upconversion detection of long-wave infrared radiation from a quantum cascade laser," Opt. Mater. Express, vol. 8, no. 5, pp. 1313-1321, May 2018.

[68] J. W. Goodman, Introduction to Fourier Optics. Roberts and Company Publishers, 2005.

[69] C. Pedersen, Q. Hu, L. Hogstedt, P. Tidemand-Lichtenberg, and J. S. Dam, "Noncollinear upconversion of infrared light," Opt. Express, vol. 22, no. 23, pp. $28027-$ 28036, Nov. 2014.

[70] C. Pedersen, E. Karamehmedović, J. S. Dam, and P. Tidemand-Lichtenberg, "Enhanced 2D-image upconversion using solid-state lasers," Opt. Express, vol. 17, no. 23, p. 20885, Nov. 2009.

[71] R. Andrews, "IR image parametric up-conversion," IEEE J. Quantum Electron., vol. 6, no. 1, pp. 68-80, Jan. 1970.

[72] J. Baumert and P. Gunter, "Noncritically Phase-Matched Sum Frequency Generation and Image Upconversion in Knbo3 Crystals," Appl. Phys. Lett., vol. 50, no. 10, pp. 554-556, Mar. 1987.

[73] "Covesion Ltd. - Material properties of Lithium Niobate." [Online]. Available: https://www.covesion.com/support/covesion-guide-to-ppln/material-properties-oflithium-niobate.html. [Accessed: 13-Jul-2018]. 
[74] P. A. Franken and J. F. Ward, "Optical Harmonics and Nonlinear Phenomena," Rev. Mod. Phys., vol. 35, no. 1, pp. 23-39, Jan. 1963.

[75] D. Feng et al., "Enhancement of second-harmonic generation in $\mathrm{LiNbO}_{3}$ crystals with periodic laminar ferroelectric domains," Appl. Phys. Lett., vol. 37, no. 7, pp. 607-609, Oct. 1980.

[76] L. Gordon et al., "Diffusion-bonded stacked GaAs for quasiphase-matched secondharmonic generation of a carbon dioxide laser," Electron. Lett., vol. 29, no. 22, pp. 1942-1944, Oct. 1993.

[77] S. Zhu et al., "LiTaO3 crystal periodically poled by applying an external pulsed field," $J$. Appl. Phys., vol. 77, no. 10, pp. 5481-5483, May 1995.

[78] B. Yan, Y. Wang, and Y. Qi, "Simultaneous three wavelength mid-IR laser by utilizing MgO:PPLN crystal," in AOPC 2017: Laser Components, Systems, and Applications, 2017, vol. 10457, p. 104573F.

[79] L. A. Pomeranz, P. G. Schunemann, D. J. Magarrell, J. C. McCarthy, K. T. Zawilski, and D. E. Zelmon, "1-micron-pumped OPO based on orientation-patterned GaP," in Nonlinear Frequency Generation and Conversion: Materials, Devices, and Applications $X I V, 2015$, vol. 9347 , p. $93470 \mathrm{~K}$.

[80] T. Skauli et al., "Measurement of the nonlinear coefficient of orientation-patterned GaAs and demonstration of highly efficient second-harmonic generation," Opt. Lett., vol. 27, no. 8, pp. 628-630, Apr. 2002.

[81] H. Pan and H. Zeng, "Efficient and stable single-photon counting at $1.55 \mu \mathrm{m}$ by intracavity frequency upconversion," Opt. Lett., vol. 31, no. 6, pp. 793-795, Mar. 2006.

[82] R. Tang, W. Wu, X. Li, H. Pan, H. Zeng, and E. Wu, "Low-Noise Infrared Spectroscopy via Tunable Frequency Upconversion at Single-Photon Level," Ieee Photonics Technol. Lett., vol. 27, no. 15, pp. 1642-1645, Aug. 2015.

[83] H. Pan, H. Dong, H. Zeng, and W. Lu, "Efficient single-photon counting at $1.55 \mu \mathrm{m}$ by intracavity frequency upconversion in a unidirectional ring laser," Appl. Phys. Lett., vol. 89, no. 19, p. 191108, Nov. 2006.

[84] M. Mancinelli et al., "Mid-infrared coincidence measurements on twin photons at room temperature," Nat. Commun., vol. 8, p. 15184, May 2017.

[85] G.-L. Shentu et al., "Upconversion detection near $2 \mu \mathrm{m}$ at the single photon level," Opt. Lett., vol. 38, no. 23, pp. 4985-4987, 12-01 2013.

[86] A. P. Vandevender and P. G. Kwiat, "High efficiency single photon detection via frequency up-conversion," J. Mod. Opt., vol. 51, no. 9-10, pp. 1433-1445, Jun. 2004.

[87] A. Barh, C. Pedersen, and P. Tidemand-Lichtenberg, "Ultra-broadband mid-wave-IR upconversion detection," Opt. Lett., vol. 42, no. 8, pp. 1504-1507, Apr. 2017.

[88] Q. Hu, J. S. Dam, C. Pedersen, and P. Tidemand-Lichtenberg, "High-resolution mid-IR spectrometer based on frequency upconversion," Opt. Lett., vol. 37, no. 24, pp. 52325234, Dec. 2012.

[89] T. A. Johnson and S. A. Diddams, "Mid-infrared upconversion spectroscopy based on a Yb:fiber femtosecond laser," Appl. Phys. B, vol. 107, no. 1, pp. 31-39, Apr. 2012.

[90] J. S. Dam, C. Pedersen, and P. Tidemand-Lichtenberg, "High-resolution twodimensional image upconversion of incoherent light," Opt. Lett., vol. 35, no. 22, pp. 3796-3798, Nov. 2010.

[91] D. Ceus et al., "Phase closure retrieval in an infrared-to-visible upconversion interferometer for high resolution astronomical imaging," Opt. Express, vol. 19, no. 9, pp. 8616-8624, Apr. 2011.

[92] C. D. Brewer, B. D. Duncan, P. S. Maciejewski, S. M. Kirkpatrick, and E. A. Watson, "Space-bandwidth product enhancement of a monostatic, multiaperture infrared image upconversion ladar receiver incorporating periodically poled LiNbO3," Appl. Opt., vol. 41, no. 12, pp. 2251-2262, Apr. 2002. 
[93] N. Sanders, J. S. Dam, O. B. Jensen, P. Tidemand-Lichtenberg, and C. Pedersen, "Multispectral mid-infrared imaging using frequency upconversion," in Nonlinear Frequency Generation and Conversion: Materials, Devices, and Applications Xii, vol. 8604, K. L. Vodopyanov, Ed. Bellingham: Spie-Int Soc Optical Engineering, 2013, p. UNSP 86040R.

[94] L. M. Kehlet, N. Sanders, P. Tidemand-Lichtenberg, J. S. Dam, and C. Pedersen, "Infrared hyperspectral upconversion imaging using spatial object translation," Opt. Express, vol. 23, no. 26, pp. 34023-34028, Dec. 2015.

[95] R. Demur et al., "Near-infrared to visible upconversion imaging using a broadband pump laser," Opt. Express, vol. 26, no. 10, pp. 13252-13263, May 2018.

[96] R. Baudoin et al., "Sum frequency generation process for a new astronomical instrument," in Nonlinear Frequency Generation and Conversion: Materials, Devices, and Applications XIII, 2014, vol. 8964, p. 89641G.

[97] M. Mathez, P. J. Rodrigo, P. Tidemand-Lichtenberg, and C. Pedersen, "Upconversion imaging using short-wave infrared picosecond pulses," Opt. Lett., vol. 42, no. 3, pp. 579-582, Feb. 2017.

[98] P. Tidemand-Lichtenberg, J. S. Dam, H. V. Andersen, L. Høgstedt, and C. Pedersen, "Mid-infrared upconversion spectroscopy," JOSA B, vol. 33, no. 11, pp. D28-D35, Nov. 2016.

[99] V. G. Dmitriev, G. G. Gurzadyan, and D. N. Nikogosyan, Handbook of Nonlinear Optical Crystals, 3rd ed. Berlin Heidelberg: Springer-Verlag, 1999.

[100] G. C. Bhar, S. Das, R. K. Route, and R. S. Feigelson, "Synchronous pulsed infrared detection in AgGaSe2 crystal using $1.318 \mathrm{mu}$ m pump," Appl. Phys. B-Lasers Opt., vol. 65, no. 4-5, pp. 471-473, Oct. 1997.

[101] L. Høgstedt, A. Fix, M. Wirth, C. Pedersen, and P. Tidemand-Lichtenberg, "Upconversion-based lidar measurements of atmospheric $\mathrm{CO}_{2}$," Opt. Express, vol. 24, no. 5, pp. 5152-5161, Mar. 2016.

[102] J. S. Dam, Q. Hu, C. Pedersen, and P. Tidemand-Lichtenberg, "Non-collinear upconversion of incoherent light: designing infrared spectrometers and imaging systems," presented at the SPIE LASE, San Francisco, California, United States, 2014, p. $89640 \mathrm{~F}$.

[103] T.-H. Wong et al., "Sensitive infrared signal detection by upconversion technique," Opt. Eng., vol. 53, no. 10, p. 107102, Oct. 2014.

[104] L. Huot, P. M. Moselund, P. Tidemand-Lichtenberg, L. Leick, and C. Pedersen, "Upconversion imaging using an all-fiber supercontinuum source," Opt. Lett., vol. 41, no. 11, pp. 2466-2469, Jun. 2016.

[105] L. Meng et al., "Upconversion detector for range-resolved DIAL measurement of atmospheric $\mathrm{CH}_{4}$," Opt. Express, vol. 26, no. 4, pp. 3850-3860, Feb. 2018.

[106] M. M. Abbas, T. Kostiuk, and K. W. Ogilvie, "Infrared upconversion for astronomical applications," Appl. Opt., vol. 15, no. 4, pp. 961-970, Apr. 1976.

[107] R. L. Pedersen, L. Høgstedt, A. Barh, L. Meng, and P. Tidemand-Lichtenberg, "Characterization of the NEP of Mid-Infrared Upconversion Detectors," IEEE Photonics Technol. Lett., vol. 31, no. 9, pp. 681-684, May 2019.

[108] L. E. Estes, R. F. Lucy, J. Gunter, and K. Duval, "Internal thermal noise in optical frequency converters," JOSA, vol. 64, no. 3, pp. 295-300, Mar. 1974.

[109] A. Barh, P. Tidemand-Lichtenberg, and C. Pedersen, "Thermal noise in mid-infrared broadband upconversion detectors," Opt. Express, vol. 26, no. 3, pp. 3249-3259, Feb. 2018.

[110] Y. C. See, S. Guha, and J. Falk, "Limits to the NEP of an intracavity $\mathrm{LiNbO}_{3}$ upconverter," Appl. Opt., vol. 19, no. 9, pp. 1415-1418, May 1980.

[111] S. Guha and J. Falk, "Theory of thermal noise upconversion in LiIO3 and LiNbO3 crystals," J. Appl. Phys., vol. 53, no. 4, pp. 2854-2861, Apr. 1982. 
[112] S. Wolf, J. Kiessling, M. Kunz, G. Popko, K. Buse, and F. Kühnemann, "Upconversion-enabled array spectrometer for the mid-infrared, featuring kilohertz spectra acquisition rates,” Opt. Express, vol. 25, no. 13, pp. 14504-14515, Jun. 2017.

[113] D. C. Burnham and D. L. Weinberg, "Observation of Simultaneity in Parametric Production of Optical Photon Pairs," Phys. Rev. Lett., vol. 25, no. 2, pp. 84-87, Jul. 1970.

[114] C. R. Phillips, J. S. Pelc, and M. M. Fejer, "Parametric processes in quasiphasematching gratings with random duty cycle errors," JOSA B, vol. 30, no. 4, pp. 982 993, Apr. 2013.

[115] C. L. Tang, "Spontaneous Emission in the Frequency Up-Conversion Process in Nonlinear Optics," Phys. Rev., vol. 182, no. 2, pp. 367-374, Jun. 1969.

[116] L. Meng, L. Høgstedt, P. Tidemand-Lichtenberg, C. Pedersen, and P. J. Rodrigo, "Enhancing the detectivity of an upconversion single-photon detector by spatial filtering of upconverted parametric fluorescence," Opt. Express, vol. 26, no. 19, pp. 24712 24722, Sep. 2018.

[117] R. A. Andrews, H. Rabin, and C. L. Tang, "Coupled Parametric Downconversion and Upconversion with Simultaneous Phase Matching," Phys. Rev. Lett., vol. 25, no. 9, pp. 605-608, Aug. 1970.

[118] J. S. Pelc et al., "Long-wavelength-pumped upconversion single-photon detector at $1550 \mathrm{~nm}$ : performance and noise analysis," Opt. Express, vol. 19, no. 22, pp. 2144521456, Oct. 2011.

[119] P. S. Kuo, J. S. Pelc, C. Langrock, and M. M. Fejer, "Using temperature to reduce noise in quantum frequency conversion," Opt. Lett., vol. 43, no. 9, pp. 2034-2037, May 2018.

[120] R. C. Miller, "Optical second harmonic generation in piezoelectric crystals," Appl. Phys. Lett., vol. 5, no. 1, pp. 17-19, Jul. 1964.

[121] G. P. Arumov, É. S. Voronin, Y. A. Il'inskii, V. E. Prokopenko, and V. S. Solomatin, "Investigation of the angular resolution in the conversion of images from $\lambda=10 \mu$ to visible in proustite," Sov. J. Quantum Electron., vol. 4, no. 9, p. 1163, 1975.

[122] L. I. Isaenko and A. P. Yelisseyev, "Recent studies of nonlinear chalcogenide crystals for the mid-IR," Semicond. Sci. Technol., vol. 31, no. 12, p. 123001, 2016.

[123] G. Bhar, S. Das, U. Chatterjee, R. Feigelson, and R. Route, "Synchronous and Noncollinear Infrared Upconversion in Aggas2," Appl. Phys. Lett., vol. 54, no. 16, pp. 1489-1491, Apr. 1989.

[124] R. Nakamura, Y. Inagaki, H. Hata, N. Hamada, N. Umemura, and T. Kamimura, "Wide-bandgap nonlinear crystal LiGaS2 for femtosecond mid-infrared spectroscopy with chirped-pulse upconversion," Appl. Opt., vol. 55, no. 33, pp. 9365-9369, Nov. 2016.

[125] S. Junaid et al., "Mid-infrared upconversion based hyperspectral imaging," Opt. Express, vol. 26, no. 3, pp. 2203-2211, Feb. 2018.

[126] L. Isaenko et al., "LiInS2: A new nonlinear crystal for the mid-IR," Mater. Sci. Semicond. Process., vol. 4, no. 6, pp. 665-668, Dec. 2001.

[127] V. V. Badikov et al., "Crystal growth and characterization of new quaternary chalcogenide nonlinear crystals for the mid-IR: $\mathrm{BaGa}_{2} \mathrm{GeS}_{6}$ and $\mathrm{BaGa}_{2} \mathrm{GeSe}_{6}, "$ Opt. Mater. Express, vol. 6, no. 9, pp. 2933-2938, Sep. 2016.

[128] A. Barh, L. Høgstedt, P. Tidemand-Lichtenberg, and C. Pedersen, "Mid-Infrared (6 $10 \mu \mathrm{m})$ upconversion in LiInS2 using $1064 \mathrm{~nm}$ CW pump," in Conference on Lasers and Electro-Optics (2018), paper SM4D.4, 2018, p. SM4D.4.

[129] W. A. Wood, D. W. Lambert, and D. V. Kuksenkov, "Modeling of Thermal Lensing in Nonlinear Optical Crystals," in Nonlinear Optics: Materials, Fundamentals and Applications (2007), paper WC8, 2007, p. WC8. 
[130] K. F. Hulme, O. Jones, P. H. Davies, and M. V. Hobden, "Synthetic proustite (Ag3AsS3): A new crystal for optical mixing," Appl. Phys. Lett., vol. 10, no. 4, pp. 133135, 02-15 1967.

[131] "Eksma Optics." [Online]. Available: http://eksmaoptics.com/. [Accessed: 08-Aug2018].

[132] F. Devaux, A. Mosset, E. Lantz, S. Monneret, and H. Le Gall, "Image upconversion from the visible to the UV domain: application to dynamic UV microstereolithography," Appl. Opt., vol. 40, no. 28, pp. 4953-4957, Oct. 2001.

[133] O. Rodenko, P. Tidemand-Lichtenberg, and C. Pedersen, "Low repetition rate 343 nm passively Q-switched solid-state laser for time-resolved fluorescence spectroscopy," Opt. Express, vol. 26, no. 16, pp. 20614-20621, Aug. 2018.

[134] "CASTECH Inc." [Online]. Available: http://www.castech.com/products.aspx?bid=3\&selectIndex=0. [Accessed: 03-Oct-2018].

[135] P. M. Vaughan and R. Trebino, "Optical-parametric-amplification imaging of complex objects," Opt. Express, vol. 19, no. 9, pp. 8920-8929, Apr. 2011.

[136] L. Pattelli, R. Savo, M. Burresi, and D. S. Wiersma, "Spatio-temporal visualization of light transport in complex photonic structures," Light Sci. Appl., vol. 5, no. 5, p. e16090, May 2016.

[137] C. Doulé, T. Lépine, P. Georges, and A. Brun, "Video rate depth-resolved twodimensional imaging through turbid media by femtosecond parametric amplification," Opt. Lett., vol. 25, no. 5, pp. 353-355, Mar. 2000.

[138] K. Morishita, N. Kawaguchi, and T. Okada, "Quality in upconversion imaging of infrared degenerate four-wave mixing spectroscopy," Opt. Commun., vol. 184, no. 5-6, pp. 443-450, Oct. 2000.

[139] E. Lantz and F. Devaux, "Parametric amplification of images: from time gating to noiseless amplification," IEEE J. Sel. Top. Quantum Electron., vol. 14, no. 3, pp. 635647, May 2008.

[140] J. D. Bierlein and H. Vanherzeele, "Potassium titanyl phosphate: properties and new applications," JOSA B, vol. 6, no. 4, pp. 622-633, Apr. 1989.

[141] Y. Furukawa et al., "Investigation of bulk laser damage threshold of lithium niobate single crystals by $Q$-switched pulse laser," J. Appl. Phys., vol. 69, no. 5, pp. 3372-3374, Mar. 1991.

[142] “Crystals-mt-berlin.” [Online]. Available: http://www.mtberlin.com/frames_cryst/crystals_frameset1.htm. [Accessed: 24-Oct-2018].

[143] S. Fossier et al., "Optical, vibrational, thermal, electrical, damage, and phasematching properties of lithium thioindate," JOSA B, vol. 21, no. 11, pp. 1981-2007, Nov. 2004.

[144] L. Isaenko et al., "LiInSe2: A biaxial ternary chalcogenide crystal for nonlinear optical applications in the midinfrared," J. Appl. Phys., vol. 91, no. 12, pp. 9475-9480, May 2002.

[145] V. Badikov et al., "Phase-Matching Properties of BaGa4S7 and BaGa4Se7: WideBandgap Nonlinear Crystals for the Mid-Infrared," in Advances in Optical Materials (2011), paper JWB4, 2011, p. JWB4.

[146] F. Yang et al., "Midinfrared optical parametric amplifier with $6.4-11 \mu \mathrm{m}$ range based on BaGa4Se7," IEEE Photonics Technol. Lett., vol. 27, no. 10, pp. 1100-1103, 052015.

[147] J. Yao et al., "BaGa4Se7: A new congruent-melting IR nonlinear optical material," Inorg. Chem., vol. 49, no. 20, pp. 9212-9216, Oct. 2010.

[148] D. V. Badikov et al., "Sum-frequency generation of Q-switched CO laser radiation in BaGa2GeSe6 and GaSe nonlinear crystals," Opt. Quantum Electron., vol. 50, no. 6, p. 243, May 2018.

[149] J. Wei, S. C. Kumar, H. Ye, P. G. Schunemann, and M. Ebrahim-Zadeh, "Performance characterization of mid-infrared difference-frequency-generation in 
orientation-patterned gallium phosphide," Opt. Mater. Express, vol. 8, no. 3, pp. 555567, Mar. 2018.

[150] A. Pratap Singh, A. Kapoor, K. N. Tripathi, and G. Rvindra Kumar, "Thermal and mechanical damage of GaAs in picosecond regime," Opt. Laser Technol., vol. 33, no. 6, pp. 363-369, Sep. 2001.

[151] R. Demur, A. Grisard, L. Morvan, E. Lallier, N. Treps, and C. Fabre, "High sensitivity narrowband wavelength mid-infrared detection at room temperature," Opt. Lett., vol. 42, no. 10, pp. 2006-2009, May 2017.

[152] P. G. Schunemann, D. J. Magarrell, and L. Mohnkern, "Growth of engineered QPM structures in orientation-patterned gallium arsenide and gallium phosphide (Conference Presentation)," in Nonlinear Frequency Generation and Conversion: Materials and Devices XVIII, 2019, vol. 10902, p. 109020H.

[153] W. C. Chiou and F. P. Pace, "Parametric Image Upconversion of 10.6- $\mu \mathrm{m}$ Illuminated Objects," Appl. Phys. Lett., vol. 20, no. 1, pp. 44-47, Jan. 1972.

[154] F. L. Schow, A. Riazi, O. P. Gandhi, and R. W. Grow, "Spatial resolution of an intracavity image upconverter," Appl. Phys. Lett., vol. 38, no. 10, pp. 757-758, May 1981.

[155] J. E. Geusic, H. J. Levinstein, S. Singh, R. G. Smith, and L. G. Van Uitert, "Continuous 0.532- $\mu$ solid-state source using Ba2NaNb5O15," Appl. Phys. Lett., vol. 12, no. 9, pp. 306-308, May 1968.

[156] W. Chiou and F. Pace, "Research on Imaging Properties of Optical Parametric Upconverter," p. 171.

[157] L. Meng, L. Høgstedt, P. Tidemand-Lichtenberg, C. Pedersen, and P. J. Rodrigo, "GHz-bandwidth upconversion detector using a unidirectional ring cavity to reduce multilongitudinal mode pump effects," Opt. Express, vol. 25, no. 13, pp. 14783-14794, Jun. 2017.

[158] R. Riedel et al., "Thermal properties of borate crystals for high power optical parametric chirped-pulse amplification," Opt. Express, vol. 22, no. 15, p. 17607, Jul. 2014.

[159] S. Dupont, P. M. Moselund, L. Leick, J. Ramsay, and S. R. Keiding, "Up-conversion of a megahertz mid-IR supercontinuum," JOSA B, vol. 30, no. 10, pp. 2570-2575, Oct. 2013.

[160] K. Huang, X. Gu, H. Pan, E. Wu, and H. Zeng, "Synchronized Fiber Lasers for Efficient Coincidence Single-Photon Frequency Upconversion," IEEE J. Sel. Top. Quantum Electron., vol. 18, no. 2, pp. 562-566, Mar. 2012.

[161] S. Wolf, T. Trendle, J. Kiessling, J. Herbst, K. Buse, and F. Kühnemann, "Self-gated mid-infrared short pulse upconversion detection for gas sensing," Opt. Express, vol. 25, no. 20, p. 24459, Oct. 2017.

[162] L. Huot, P. M. Moselund, L. Leick, P. Tidemand-Lichtenberg, and C. Pedersen, "Broadband upconversion imaging around $4 \mathrm{mu}$ m using an all-fiber supercontinuum source," in Nonlinear Frequency Generation and Conversion: Materials and Devices $X v i$, vol. 10088, K. L. Vodopyanov and K. L. Schepler, Eds. Bellingham: Spie-Int Soc Optical Engineering, 2017, p. UNSP 100880J.

[163] K. Huang, X. Gu, H. Pan, E Wu, and H. Zeng, "Few-photon-level two-dimensional infrared imaging by coincidence frequency upconversion," Appl. Phys. Lett., vol. 100, no. 15, p. 151102, Apr. 2012.

[164] M. Mathez, C. Pedersen, P. J. Rodrigo, and P. Tidemand-Lichtenberg, "Investigation of mid-IR picosecond image upconversion," 2017, p. 100880L.

[165] L. Huot, P. M. Moselund, P. Tidemand-Lichtenberg, and C. Pedersen, "Electronically delay-tuned upconversion cross-correlator for characterization of midinfrared pulses," Opt. Lett., vol. 43, no. 12, pp. 2881-2884, Jun. 2018. 
[166] J. S. Dam, C. Pedersen, and P. Tidemand-Lichtenberg, "Theory for upconversion of incoherent images," Opt. Express, vol. 20, no. 2, pp. 1475-1482, Jan. 2012.

[167] J. S. Dam, C. Pedersen, and P. Tidemand-Lichtenberg, "Improved space bandwidth product in image upconversion," in Nonlinear Frequency Generation and Conversion: Materials, Devices, and Applications Xi, vol. 8240, K. L. Vodopyanov, Ed. Bellingham: Spie-Int Soc Optical Engineering, 2012, p. 82400F.

[168] S. Junaid et al., "Video-rate, mid-infrared hyperspectral upconversion imaging," Optica, vol. 6, no. 6, pp. 702-708, Jun. 2019.

[169] H. Maestre, A. J. Torregrosa, and J. Capmany, "Application of a temperaturegradient PPLN crystal for IR image up-conversion," in Conference on Lasers and Electro-Optics (2016), paper JTu5A.61, 2016, p. JTu5A.61.

[170] H. Maestre, A. J. Torregrosa, C. R. Fernández-Pousa, and J. Capmany, "IR-tovisible image upconverter under nonlinear crystal thermal gradient operation," Opt. Express, vol. 26, no. 2, pp. 1133-1144, Jan. 2018.

[171] H. Maestre, A. J. Torregrosa, and J. Capmany, "IR Image upconversion using bandlimited ASE illumination fiber sources," Opt. Express, vol. 24, no. 8, pp. 8581-8593, Apr. 2016.

[172] H. Maestre, A. J. Torregrosa, and J. Capmany, "IR Image Upconversion Under Dual-Wavelength Laser Illumination," IEEE Photonics J., vol. 8, no. 6, pp. 1-8, Dec. 2016.

[173] A. J. Torregrosa, H. Maestre, and J. Capmany, "Intra-cavity upconversion to $631 \mathrm{~nm}$ of images illuminated by an eye-safe ASE source at 1550 nm," Opt. Lett., vol. 40, no. 22, pp. 5315-5318, 11-15 2015.

[174] J. F. Weller and R. A. Andrews, "Resolution measurements in parametric upconversion of images," Opto-Electron., vol. 2, no. 3, pp. 171-176, Aug. 1970.

[175] K. F. Hulme and J. Warner, "Theory of Thermal Imaging Using Infrared to Visible Image Up-Conversion," Appl. Opt., vol. 11, no. 12, pp. 2956-2964, Dec. 1972.

[176] W. Chiou, "Geometric Optics Theory of Parametric Image Upconversion," J. Appl. Phys., vol. 42, no. 5, pp. 1985-1993, Apr. 1971.

[177] T. Mishima and I. Sakuraba, "Analysis of Chromatic Aberrations in Parametric Image-Upconversion Systems," p. 14.

[178] R. Tang, W. Wu, X. Li, H. Pan, E. Wu, and H. Zeng, "Two-dimensional infrared and mid-infrared imaging by single-photon frequency upconversion (vol 62, pg 1126, 2015)," J. Mod. Opt., vol. 62, no. 20, pp. 1755-1755, 2015.

[179] T. R. E. Gurski, "Astronomical demonstration of an infrared upconverter," Nature, Jun. 1974.

[180] Y.-P. Tseng, P. Bouzy, C. Pedersen, N. Stone, and P. Tidemand-Lichtenberg, "Upconversion raster scanning microscope for long-wavelength infrared imaging of breast cancer microcalcifications," Biomed. Opt. Express, vol. 9, no. 10, pp. 4979-4987, Oct. 2018.

[181] S. Junaid, P. Tidemand-Lichtenberg, and C. Pedersen, "Upconversion based spectral imaging in 6 to $8 \mathrm{mu}$ m spectral regime," in Nonlinear Frequency Generation and Conversion: Materials and Devices Xvi, vol. 10088, K. L. Vodopyanov and K. L. Schepler, Eds. Bellingham: Spie-Int Soc Optical Engineering, 2017, p. UNSP 100880I.

[182] R. V. Roussev, C. Langrock, J. R. Kurz, and M. M. Fejer, "Periodically poled lithium niobate waveguide sum-frequency generator for efficient single-photon detection at communication wavelengths," Opt. Lett., vol. 29, no. 13, pp. 1518-1520, Jul. 2004.

[183] P. S. Kuo, O. Slattery, Y.-S. Kim, J. S. Pelc, M. M. Fejer, and X. Tang, "Spectral response of an upconversion detector and spectrometer," Opt. Express, vol. 21, no. 19, pp. 22523-22531, Sep. 2013.

[184] L. Gampel and F. Johnson, "IR image detection by CW parametric up-conversion to the visible," IEEE J. Quantum Electron., vol. 4, no. 5, pp. 354-354, May 1968. 
[185] Y. P. Tseng, P. Bouzy, N. Stone, C. Pedersen, and P. Tidemand-Lichtenberg, "Long wavelength identification of microcalcifications in breast cancer tissue using a quantum cascade laser and upconversion detection," in Biomedical Vibrational Spectroscopy 2018: Advances in Research and Industry, 2018, vol. 10490, p. 104900F.

[186] N. M. Israelsen et al., "Real-time high-resolution mid-infrared optical coherence tomography," Light Sci. Appl., vol. 8, no. 1, p. 11, Jan. 2019.

[187] P. A. Laferriere, C. J. Wetterer, L. P. Schelonka, and M. A. Kramer, "Spatialfrequency selection using downconversion optical parametric amplification," J. Appl. Phys., vol. 65, no. 9, pp. 3347-3350, May 1989.

[188] E. Lantz and F. Devaux, "Parametric amplification of images," Quantum Semiclassical Opt. J. Eur. Opt. Soc. Part B, vol. 9, no. 2, p. 279, 1997.

[189] J. C. Delagnes, P. Mounaix, and L. Canioni, "Nonlinear holographic imaging of terahertz radiation," in Nonlinear Frequency Generation and Conversion: Materials, Devices, and Applications X, vol. 7917, K. L. Vodopyanov, Ed. Bellingham: Spie-Int Soc Optical Engineering, 2011, p. 79170D.

[190] S. Fan et al., "Diffraction-limited real-time terahertz imaging by optical frequency up-conversion in a DAST crystal," Opt. Express, vol. 23, no. 6, pp. 7611-7618, Mar. 2015.

[191] W. Zhao et al., "Efficient optical image amplifier using periodically poled lithium niobate," Appl. Opt., vol. 54, no. 31, pp. 9172-9176, Nov. 2015.

[192] J. Yang et al., "Image enhancement based on optical parametric amplification," in Fourth International Symposium on Laser Interaction with Matter, 2017, vol. 10173, p. $101731 \mathrm{~V}$.

[193] Y. Jiang and Y. J. Ding, "Efficient upconversion of coherent radiation at $10.26 \mu \mathrm{m}$," in 2008 Conference on Lasers and Electro-Optics and 2008 Conference on Quantum Electronics and Laser Science, 2008, pp. 1-2.

[194] X. Qiu, F. Li, W. Zhang, Z. Zhu, and L. Chen, "Spiral phase contrast imaging in nonlinear optics: seeing phase objects using invisible illumination," Optica, vol. 5, no. 2, pp. 208-212, Feb. 2018.

[195] H. Liu, H. Li, Y. Zheng, and X. Chen, "Nonlinear frequency conversion and manipulation of vector beams," Opt. Lett., vol. 43, no. 24, pp. 5981-5984, Dec. 2018.

[196] B. Chalopin, A. Chiummo, C. Fabre, A. Maître, and N. Treps, "Frequency doubling of low power images using a self-imaging cavity," Opt. Express, vol. 18, no. 8, pp. 8033-8042, Apr. 2010.

[197] A. J. Torregrosa, H. Maestre, M. L. Rico, and J. Capmany, “Compact selfilluminated image upconversion system based on intracavity second-harmonic generation," Opt. Lett., vol. 43, no. 20, p. 5050, Oct. 2018.

[198] S. Tan, X. Wei, B. Li, Q. T. K. Lai, K. K. Tsia, and K. K. Y. Wong, "Ultrafast optical imaging at $2.0 \mu \mathrm{m}$ through second-harmonic-generation-based time-stretch at $1.0 \mu \mathrm{m}$," Opt. Lett., vol. 43, no. 16, pp. 3822-3825, 08-15 2018.

[199] A. V. Gainer, G. V. Krivoshchekov, S. V. Kruglov, V. V. Lebedev, and S. I. Marennikov, "Investigation of wide-angle image conversion systems," Sov. J. Quantum Electron., vol. 1, no. 6, p. 670, 1972.

[200] C. Yan and J. Diels, "Imaging with Femtosecond Pulses," Appl. Opt., vol. 31, no. 32, pp. 6869-6873, Nov. 1992.

[201] G. W. Faris and M. Banks, "Upconverting time gate for imaging through highly scattering media," Opt. Lett., vol. 19, no. 22, pp. 1813-1815, Nov. 1994.

[202] K. Morishita, Y. Higuchi, and T. Okada, "Infrared laser spectroscopic imaging based on degenerate four-wave-mixing spectroscopy combined with frequency-upconversion detection," Opt. Lett., vol. 24, no. 10, pp. 688-690, May 1999. 
[203] Q. Zhou, K. Huang, H. Pan, E. Wu, and H. Zeng, "Ultrasensitive mid-infrared upconversion imaging at few-photon level," Appl. Phys. Lett., vol. 102, no. 24, p. 241110 , Jun. 2013.

[204] S. Fan et al., "Real-time terahertz wave imaging by nonlinear optical frequency upconversion in a 4-dimethylamino-N'-methyl-4'-stilbazolium tosylate crystal," Appl. Phys. Lett., vol. 104, no. 10, p. 101106, Mar. 2014.

[205] N. Sanders, J. S. Dam, P. Tidemand-Lichtenberg, and C. Pedersen, "Near Diffraction limited mid-IR Spectromicroscopy, Using Frequency Upconversion," in Nonlinear Frequency Generation and Conversion: Materials, Devices, and Applications Xiii, vol. 8964, K. L. Vodopyanov, Ed. Bellingham: Spie-Int Soc Optical Engineering, 2014, p. $89641 \mathrm{~L}$

[206] N. Huang, H. Liu, Z. Wang, J. Han, and S. Zhang, "Femtowatt incoherent image conversion from mid-infrared light to near-infrared light," Laser Phys., vol. 27, no. 3, pp. 1-5, Mar. 2017.

[207] L. Huot, P. M. Moselund, P. Tidemand-Lichtenberg, and C. Pedersen, "Pulsed upconversion imaging of mid-infrared supercontinuum light using an electronically synchronized pump laser," Appl. Opt., vol. 58, no. 2, pp. 244-249, Jan. 2019.

[208] A. A. S, C. F. O’Donnell, S. C. Kumar, M. Ebrahim-Zadeh, P. TidemandLichtenberg, and C. Pedersen, "Mid-infrared upconversion imaging using femtosecond pulses," Photonics Res., vol. 7, no. 7, pp. 783-791, Jul. 2019.

[209] H. Mahr and M. D. Hirsch, "An optical up-conversion light gate with picosecond resolution," Opt. Commun., vol. 13, no. 2, pp. 96-99, Feb. 1975.

[210] L. A. Hallidy and M. R. Topp, "Picosecond luminescence detection using type-II phase-matched frequency conversion," Chem. Phys. Lett., vol. 46, no. 1, pp. 8-14, Feb. 1977.

[211] J. Shah, "Ultrafast luminescence spectroscopy using sum frequency generation," IEEE J. Quantum Electron., vol. 24, no. 2, pp. 276-288, Feb. 1988.

[212] H. Chosrowjan, S. Taniguchi, and F. Tanaka, "Ultrafast fluorescence upconversion technique and its applications to proteins," Febs J., vol. 282, no. 16, pp. 3003-3015, 08 2015.

[213] K. A. Briggman, L. J. Richter, and J. C. Stephenson, "Imaging and autocorrelation of ultrafast infrared laser pulses in the 3-11- $\mu \mathrm{m}$ range with silicon CCD cameras and photodiodes," Opt. Lett., vol. 26, no. 4, pp. 238-240, Feb. 2001.

[214] C. Yan and J.-C. Diels, "Two-dimensional imaging and three-dimensional reconstruction of low reflectivity surfaces by using the range-gating upconversion second-harmonic method," Appl. Opt., vol. 34, no. 16, pp. 2993-2997, Jun. 1995.

[215] O. Kuzucu, F. N. C. Wong, S. Kurimura, and S. Tovstonog, "Time-resolved singlephoton detection by femtosecond upconversion," Opt. Lett., vol. 33, no. 19, pp. 22572259, Oct. 2008.

[216] M. Allgaier et al., "Streak camera imaging of single photons at telecom wavelength," Appl. Phys. Lett., vol. 112, no. 3, p. 031110, Jan. 2018.

[217] E. Abraham et al., "Real-time two-dimensional imaging in scattering media by use of a femtosecond $\mathrm{Cr}^{4+}$ :forsterite laser," Opt. Lett., vol. 25, no. 12, pp. 929-931, Jun. 2000.

[218] A. H. Firester, "Holography and Parametric Image Conversion: Part II," J. Appl. Phys., vol. 40, no. 12, pp. 4849-4853, Nov. 1969.

[219] E. Almeida, O. Bitton, and Y. Prior, "Nonlinear metamaterials for holography," Nat. Commun., vol. 7, Aug. 2016.

[220] R. Tang, X. Li, W. Wu, H. Pan, H. Zeng, and E. Wu, "High efficiency frequency upconversion of photons carrying orbital angular momentum for a quantum information interface," Opt. Express, vol. 23, no. 8, pp. 9796-9802, Apr. 2015. 
[221] F. Steinlechner, N. Hermosa, V. Pruneri, and J. P. Torres, "Frequency conversion of structured light," Sci. Rep., vol. 6, p. 21390, Feb. 2016.

[222] Z.-Y. Zhou et al., "Generation of light with controllable spatial patterns via the sum frequency in quasi-phase matching crystals," Sci. Rep., vol. 4, p. 5650, Jul. 2014.

[223] M. Hermes et al., "Mid-IR hyperspectral imaging for label-free histopathology and cytology," J. Opt., vol. 20, no. 2, p. 023002, 2018.

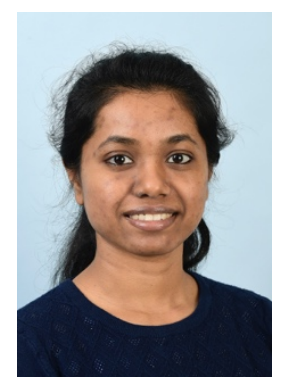

Ajanta Barh received the BSc degree from Calcutta University, Kolkata, India, in 2008, and the MSc and $\mathrm{PhD}$ degrees from Indian Institute of Technology Delhi, New Delhi, India, in 2010 and 2015, respectively, all in physics. In 2016, she joined Optical Sensor Technology group at DTU Fotonik, Department of Photonics Engineering, Technical University of Denmark as a post-doctoral fellow. In 2019, she joined the Ultrafast Laser Physics group at ETH Zurich, Department of Physics, where she is currently involved in developing ultrafast mid-infrared solid state and semiconductor laser systems for frequency metrology. She has authored /co-authored more than 40 peer-reviewed journal and conference publications. Her current research interests include mid-infrared photonics, nonlinear optics, upconversion detection, ultrafast laser development. She is a young professional member of OSA, and currently chairing the OSA-Nonlinear Optics Technical group.

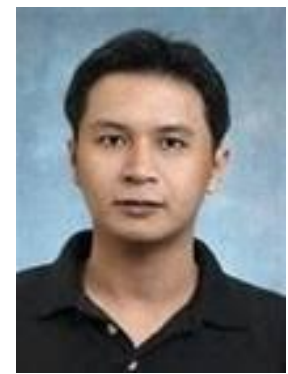

Peter John Rodrigo graduated (BSc Applied Physics in 2000 and MSc Physics in 2002) from the University of the Philippines (Diliman) and received his $\mathrm{PhD}$ in 2005 from the Niels Bohr Institute, Faculty of Science, University of Copenhagen, Denmark. He continued his research as a post-doctoral fellow at Risø National Laboratory in Denmark, where he worked on novel optical micromanipulation systems using spatial light modulators. In 2008, he joined the Optical Sensor Technology group at DTU Fotonik, Department of Photonics Engineering, Technical University of Denmark, where he developed semiconductor laser based lidar systems for wind turbine control applications. He is currently a Senior Researcher at DTU Fotonik. His research activities include development and application of upconversion detection and imaging systems and lidar based sensors.

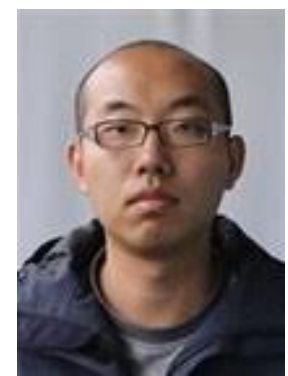

Lichun Meng received his MSc from the Abbe School of Photonics, Germany, in 2015. In 2019, he received his PhD in photonics from DTU Fotonik, Department of Photonics Engineering, Danmarks Tekniske Universitet (DTU), Denmark. Now he is an industrial postdoctoral researcher at Windar Photonics A/S - a Danish start-up company manufacturing and selling wind-sensing lidars. His research interests involve frequency upconversion detection and lidar techniques for wind speed measurement. 


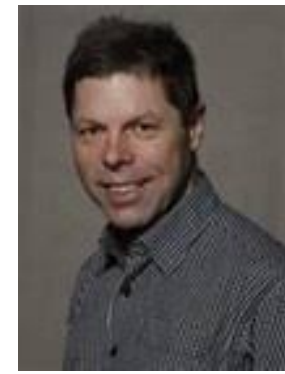

Christian Pedersen received his MSc from Technical University of Denmark (DTU) in 1991. In 1995, he received his Industrial PhD from the Department of Physics within the field of nonlinear optics and solid state lasers. From 1996 to 2004, he worked in two start-up laser companies. In 2005 to 2008, he worked at Risø National Laboratory as head of the programme within optical sensor technology. In 2008, Risø National Laboratory became a part of DTU. Since then he has been employed by DTU Fotonik, Department of Photonics Engineering, appointed professor in 2019 within the field of photonics sensors.

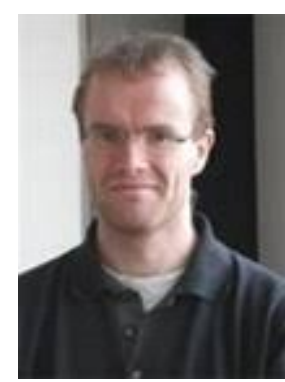

Peter Tidemand-Lichtenberg received his MSc from the Technical University of Denmark in 1993. In 1996 he received his $\mathrm{PhD}$ from the Department of Physics, DTU. After completion of the PhD, he worked in a private startup company for 5 years, developing novel compact light sources based on diode pumped solid state lasers and second order nonlinear frequency conversion. In 2002, he returned to DTU Physics, and in 2009 he was transferred to the Optical Sensor Technology group at DTU Fotonik. His research activities mainly relate to ultra-sensitive light detection systems for the mid-IR range, approaching single-photon imaging. His work has a strong focus on real life applications, generally within spectroscopy for gas sensing or medical diagnostics. 